\title{
A catalogue of butterflies (Lepidoptera, Rhopalocera) of Azerbaijan
}

\author{
N.Y. Snegovaya, V.A. Petrov \\ Zoological Institute National Academy of Science of Azerbaijan \\ A. Abbaszade St. 1128, Baky, Azerbaijan, E-mail: snegovaya@yahoo.com \\ National Museum of Georgia \\ Rustaveli Ave.3, Tbilisi, Georgia, E-mail:valerapetrovi@mail.ru
}

\begin{abstract}
An annotated list of butterflies from Azerbaijan, containing 280 species, is presented. The classification and nomenclature in this work are accepted according to the key of Korb \& Bolshakov (2016). The full list of species composition of butterflies of Azerbaijan numbering 280 species for today is presented for the first time, and it was supplemented by new materials collected by the first author from 2012 to 2019. We came up with the list of the total species composition of butterflies in Azerbaijan, which currently includes 280 species from 6 families (Hesperiidae - 37, Papilionidae - 10, Pieridae - 28, Lycaenidae - 100, Riodinidae - 1, Nimphalidae - 104) for today was compiled. We record Papilio demoleus demoleus Linnaeus, 1758 as a new species for the fauna of the Caucasus. Our results will help to continue biodiversity study and expand the information on the distribution of the butterflies (Papilionoformes) in Azerbaijan and the Caucasus.
\end{abstract}

Key words: butterflies; Azerbaijan; catalogue; Lepidoptera

Rhopalocera or butterflies are one of the popular groups of insects, which are an important component of terrestrial biocenoses. The fauna of Lepidoptera of Azerbaijan has attracted the attention of lepidopterists for a long time. The first butterfly researcher in Azerbaijan was E.Menetries (Menetries, 1832, 1855, 1859). Further studies of Lepidoptera in Azerbaijan are associated with the names of such famous entomologists as J. Lederer (Lederer, 1864, 1870), H. Christoff (Christoff, 1874, 1877, 1886, 1889, 1893), G. Radde (Radde, 1899), N.M. Romanov (1884-1887), V.V. Sovinsky (1905), A.A. Yakhontov (1911, 1913), and others. In Soviet times, such well-known lepidopterists as M.A. Ryabov (1958), L.A. Shelyuzhko (1925, 1929, 1937), E.A. Didmanidze (1979, 2004), Y.P. Korshunov (1972), Yu.P. Nekrutenko (1968, 1972, 1977, 1980, 1985, 1989, 1990) were studying the Lepidoptera of Azerbaijan. Later, information on species from Azerbaijan was presented in the works of P.V. Bogdanov (2007, 2008), A.V. Dantchenko (2000, 2004), P.Y. Gorbunov (2001), S.K. Korb (2005), V.A. Lukhtanov (1987, 1989, 2000, 2015), V.S. Murzin (2000), V.V. Tschikolovets (2011, 2012), V.K. Tuzov (1993, 1997, 2000) and others.

The systematic study of the butterflies on the territory of Azerbaijan is closely connected with the name of the well-known entomologist Rustam Mamed-Emin oglu Effendi (1934-1991). As a result of his research on the territory of the republic, 213 species of diurnal lepidoptera were identified, for most species, their biological and ecological features were revealed, distribution maps of endemic and rare species are given; vertical distribution, relative occurrence, and ecological adaptivity are established. Unfortunately, most of the information on butterflies is given in his dissertation work, and in articles and abstract of the dissertation, the data on the species composition, biology, ecology and distribution of each species are not given. Therefore, data on many species were not available to this lepidopterist and were not taken into account when writing catalogs and papers on the Caucasus. This catalog was compiled on the basis of literary sources of various authors working in the Caucasus region and adjacent territories, as well as on the basis of the dissertation work of R. M-E. Effendi (1970).

The classification and nomenclature in this work are accepted according to the key of Korb \& Bolshakov (2016).

The novelty of the work lies in the fact that actually, an available full list of species composition of butterflies of Azerbaijan numbering 280 species for today is presented for the first time, and new materials collected by the first author from 2012 to 2019 supplemented it.

1. Erynnis tages tages (Linnaeus, 1758)

Family Hesperiidae Latreille, 1809

Effendi, 1970: Thanaos tages L., everywhere: from lowlands to mountains

Didmanidze, 1979: Erynnis tages L., Turianchay (Türyançay Dövlət Təbiət Qoruğu) State Reserve, Nakhichevan AR

(Naxçıvan Muxtar Respublikası): Bilyav (Biləv), Givrak (Gıvrak)

Tuzov, 1993: Erynnis tages unicolor Freyer, 1852, Talysh (Talış); Armenian plateau 
Devyatkin, 1997: Erynnis tages L., Khatchmas distr. (Xaçmaz), loc. Nabran

Tschikolovets, Nekrutenko, 2012: Erynnis tages tages (Linnaeus, 1758), Nakhichevan, Buzgov

Korb, Bolshakov, 2016: Erynnis tages tages (Linnaeus, 1758), Azerbaijan

Material: $10^{\circ}$, Nakhichevan, along the Julfa-Ordubad highway, Julfa distr., 8.05.2018; 20'0", Shemakha, Archiman-Pirguli, near Shakhdag Milli Park, 19-20.05.2013

\section{Erynnis marloyi marloyi (Boisduval, [1834])}

Romanoff, 1884: Nisoniades Marloyi B., Ordoubad

Effendi, 1970: T.marloyi B., Nakhichevan: Ordubad, Unus; Lerik: Gosmalyan, Kyalvaz (Kəlvəz), Kyalakhan (Kələxan)

Didmanidze, 1979: Erynnis marloyi B., Nakhichevan: Bilyav (Biləv), Araks, Givrak

Tuzov, 1993: Erynnis marloi marloi (Boisduval, [1834]), Talysh; Armenian plateau

Devyatkin, 1997: Erynnis marloi marloi (Boisduval, [1834]), Nakhichevan Region: Remeshin (now Ashagi Guney) (Remeşin, now Aşağı Güney)

Didmanidze, 2004: Erynnis marloi Borisduval, 1832, Turianchay Reservation; Nakhichevan: Arax gorge near Kivrak Tschikolovets, Nekrutenko, 2012: E.marloyi marloyi (Boisduval, [1834]), Nakhichevan: Daralagez (Dərələyəz), Buzgov, Ordubad distr., Pazmara

Korb, Bolshakov, 2016: Erynnis marloyi (Boisduval, 1832), Azerbaijan

Material: 20'0', Nakhichevan, Ordubad, Agdere, 9-12.06.2017

\section{Carcharodus alceae alceae (Esper, [1780])}

Christoph, 1874: Spilothyrus Alceae Esp., Lenkoran (Lənkəran)

Christoph, 1877: Spilothyrus alceae Esp., Lenkoran

Christoph, 1886: Spilothyrus Alceae Esp., Talysch

Effendi, 1970: Carcharodus alceae Esp., everywhere in the Republic

Tuzov, 1993: Carcharodus alceae alceae Esper, 1780, Talysh; Armenian plateau

Tschikolovets, Nekrutenko, 2012: Carcharodus alceae alceae (Esper, [1780]), Nakhichevan, Ordubad; Talysh, Gosmolyan; Talysh, Zuvand, Galabin (Gələbin); Alty-Agach (Altıagaç)

Korb, Bolshakov, 2016: Carcharodus alceae (Esper, [1780]), Azerbaijan

Material: 20º', Shemakha, Pyrguli, 25.06.2015; 10', Lenkoran, Azfilial, 16-19.09.2013

\section{Carcharodus lavatherae tauricus Reverdin, 1915}

Ménétriés, 1832: Hesperia Lavatherae Esp., Talyche

Kolenati, 1846: Syricthus Lavatherae Esp., Karabagh (Garabağ); Talysch

Ménétriés, 1855: Pyrgus Lavatherae, Lenkoran

Romanoff, 1844: Spilothyrus Lavatherae Esp., Ordoubad; Adjikent (Hacıkənd) (near Gandja)

Christoph, 1886: Spilothyrus Lavatherae Esp., Talysch

Effendi, 1970: Carcharodus lavantherae Esp., everywhere in the lowlands and in the foothills

Tuzov, 1993: Carcharodus lavatherae Esper, 1780, Talysh; Armenian plateau

Devyatkin, 1997: Carcharodus lavantherae (Esper, [1783], Nakhichevan Region: Daralagezsky Mts., Buzgov

Tschikolovets, Nekrutenko, 2012: Carcharodus lavatherae tauricus Reverdin, 1915, Nakhichevan: Daralgez Mts., Kyukyu Korb, Bolshakov, 2016: Carcharodus lavatherae (Esper, [1783]), Azerbaijan

Material: $10^{2}$, Nakhichevan, Ordubad, Agdere, 22.07.2017

\section{Carcharodus stauderi ambiguous (Verity, 1925)}

Devyatkin, 1990: Carcharodus stauderi Reverdin, Nakhichevan, Negram (Nəhrəm); Talysh, Zuvand

Tuzov, 1993: Carcharodus stauderi ambigua Verity, 1925, Talysh; Armenian plateau

Devyatkin, 1997: Carcharodus stauderi ambigua Verity, 1925, Nakhichevan, Negram; Talysh, Gosmolyan

Tschikolovets, Nekrutenko, 2012: Carcharodus stauderi ambiguous (Verity, 1925), Talysh-Zuvand, Hili-Dara (Hilidərə)

Korb, Bolshakov, 2016: Carcharodus stauderi Reverdin, 1913, Azerbaijan

\section{Carcharodus flocciferus flocciferus (Zeller, 1847)}

Effendi, 1970: Carcharodus altheae $\mathrm{Hb}$, throughout the lowlands and in the foothills

Didmanidze, 1979: Carcharodus floceiferus Z. (=althea Hb.), Nakhichevan, river Araxi gorge, near state border, Kivrak, river Paragachai (Parağaçay) gorge, Mount Kapujukh (Gapıcık)

Devyatkin, 1990: Carcharodus flocciferus (Zeller), Gyandzha (Gəncə)

Didmanidze, 2004: Carcharodus flocciferus Zeller, 1847, Nakhichevan, river Araxi gorge, near state border, Kivrak, river Paragachai gorge, Mount Kapujukh

Korb, Bolshakov, 2016: Carcharodus flocciferus (Zeller, 1847), Azerbaijan

Material: 10", 19, Nakhichevan, Shakhbuz, Kyukyu, 27.07.2016 
Devjatkin, 1988: Carcharodus orientalis, Ordubad, Pazmara, Zuvand

Tuzov, 1993: Carcharodus orientalis orientalis Reverdin, 1913, Talysh; Armenian plateau

Devyatkin, 1997: Carcharodus orientalis Reverdin, 1913, Talysh

Tschikolovets, Nekrutenko, 2012: Carcharodus orientalis orientalis (Reverdin, 1913), Talysh-Zuvand, Halabyn (Gələbin)

Korb, Bolshakov, 2016: Carcharodus orientalis Reverdin, 1913, Azerbaijan

Material: 10', Lerik, Gosmalyan, 21-22.06.2017

8. Spialia orbifer orbifer (Hübner, [1823])

Alphéraky, 1876: Syrichtus Orbifer Hb., Ordoubad

Effendi, 1970: Hesperia sao Hb, Belokani (Balakən), Akh-Kimal (Axkimal); Lachin (Laçın), Zorkeshish (Zorkeşiş); Shemakha (Şamaxı)

Didmanidze, 1979: Spialia Sertorius sbsp. orbifer Hbn., Nakhichevan, Bilav, Mount Kapujukh, river Araxi gorge near

Kivrak

Tuzov, 1993: Spialia orbifer orbifer Hubner, 1802, Talysh; Armenian plateau

Didmanidze, 2004: Spialia Sertorius sbsp. orbifer Hbn., Nakhichevan, Bilav, Mount Kapujukh, river Araxi gorge near Kivrak

Tschikolovets, Nekrutenko, 2012: Spialia orbifer orbifer (Hübner, [1823]), Talysh-Zuvand, Goveri; Nakhichevan, Daralagez (Daralagöz) Mts., Buzgov

Korb, Bolshakov, 2016: Spialia orbifer (Hübner, [1823]), Azerbaijan

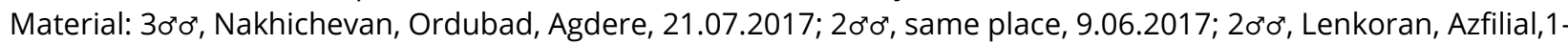

4.10.2012

9. Spialia phlomidis phlomidis (Herrich-Schaffer, [1845])

Hemming, 1932: Syrichtus phlomidis (H.-S., 1845), Ordoubad

Evans, 1949: Spialia phlomidis Herrich-Schaffer, 1845, Ordubad

Effendi, 1970: Hesperia phlomidis HS, Ordubad, Paiz (Payız); Zangelan (Zəngilan); Talish, Zuvand

De Jong, 1974: Spialia phlomidis phlomidis (Herrich-Schaffer), Ordubad

Devyatkin, 1997: Spialia phlomidis (Herrich-Schaffer, [1845]), Nakhichevan Region, Daralagezsky Mts., Buzgov

Tschikolovets, 2011: Spialia phlomidis (Herrich-Schaffer, [1845]), Nakhichevan

10. Syrichtus poggei poggei(Lederer, 1858)

Devjatkin, 1990: Muschampia poggei(Lederer, 1858), Nakhichevan, Buzgov

Devyatkin, 1997: Muschampia poggei (Lederer, 1858), Nakhichevan Region, Daralagezsky Mts., Buzgov

11. Syrichtus tessellum tessellum (Hübner, [1803])

Effendi, 1970: Hesperia tessellum Hb, Ordubad, Paiz, Unus

Didmanidze, 1979: Muschampia tesselum Hb. (= Pyrgus gigas Br.), Turianchay Reservation; Nakhichevan, Ordubad

Didmanidze, 2004: Muschampia tessellum tessel/um Hübner, 1802, Turianchay Reservation; Nakhichevan, Ordubad;

Mugan (Muğan) steppe

Korb, Bolshakov, 2016: Syrichtus tessellum (Hübner, [1803]), Azerbaijan

Material: 200'0', Yardimli, 17-20.05.2016

\section{Syrichtus tessellum tersus (Evans, 1949)}

Evans, 1949: Muschampia tessellum Sub-sp. tersa nov., Ordubad

Hesselbarth, van Oorschot \& Wagener, 1995: Muschampia tessellum tersa Evans, 1949, Nachitschewan, Nagorny Karabach (Dağlıg Garabağ)

Devyatkin, 1997: Muschampia tersa Evans, 1949, Nakhichevan, Ordubad

Tschikolovets, Nekrutenko, 2012: Muschampia nomas tersa Evans, 1949, Nakhichevan, Pazmara

13. Syrichtus proto aragonensis (De Sagarra, 1924)

Effendi, 1970: Hesperia proto Esp., Ordubad

14. Pyrgus melotis ponticus (Reverdin, 1914)

Effendi, 1970: Hesperia pontica Reverd., Shakhdag (Şaxdağ), Bazardyuzi (Bazardüzi), Dalidag (Dəlidağ), Kapudjik (Gapıcık)

Didmanidze, 1979: Pyrgus melotis Dup. sbsp. caucasica Rjab., Nakhichevan, Paragachay gorge

Tuzov, 1993: Pyrgus melotis ponticus Reverdin, 1914, Talysh; Armenian plateau

Devyatkin, 1997: Pyrgus melotis ponticus Reverdin, 1914, Kusary (now Gusar)

Didmanidze, 2004: Pyrgus melotis Duponchel, 1832 sbsp. caucasica Rjabov, Nakhichevan, Paragachay gorge

Tschikolovets, Nekrutenko, 2012: Pyrgus melotis ponticus (Reverdin, 1914), Nabran

Korb, Bolshakov, 2016: Pyrgus melotis (Duponchel, 1832), Azerbaijan

Material: $20^{\prime \prime} \sigma^{\prime \prime}$, Nakhichevan, Shakhbuz distr., between the village of Mahmudoba and Nursu, 30.05.2018 
15. Pyrgus serratulae major (Staudinger, 1878)

Romanoff, 1884: Syrichtus Serratulae Rbr., Ordoubad

Effendi, 1970: Hesperis serratulae Rbr., Babadag (Babadağ), Bazarduzi, Shakhdag

Devyatkin, 1997: Pyrgus serratulae (Rambur, [1839]), Nakhichevan

Tschikolovets, Nekrutenko, 2012: Pyrgus serratulae major (Staudinger, 1878), Nakhichevan, Ordubad distr., Pazmaraç Bitshenek; Zangezur Mts.

Korb, Bolshakov, 2016: Pyrgus serratulae (Rambur, 1839), Azerbaijan

Material: $1 \sigma^{\prime \prime}$, Shakhbuz, Nursu, 8.06.2017; 19, Massali, near Kubin and Mijankyu vill., 5.05.2014

16. Pyrgus armoricanus persicus (Reverdin, 1913)

Tuzov, 1993: Pyrgus armoricanus persicus Reverdin, 1913, Talysh; Armenian plateau

Devyatkin, 1997: Pyrgus armoricanus persicus, Nakhichevan

Tschikolovets, Nekrutenko, 2012: Pyrgus armoricanus persicus (Reverdin, 1913), Talysh, Halabin

Korb, Bolshakov, 2016: Pyrgus armoricanus (Oberthür, 1910), Azerbaijan

Material: $10^{\prime \prime}$, Nakhichevan, Ordubad, Agdere, 28-30.07.2016; 10", same place, 28.07.2016; 10", Yardimli, 17-20.05.2016; 10", Shemakha, Demirchi, 24.06.2015

\section{Pyrgus alveus alveus (Hübner, [1803])}

Effendi, 1970: Hesperia (Pyrgus) alveus Hb, throughout the lowlands and in the foothills

Tschikolovets, Nekrutenko, 2012: Pyrgus alveus alveus (Hübner, [1803]), Talysh, Lerik; Nakhichevan, Buzgov; Mingetshaur (Mingəçevir)

Korb, Bolshakov, 2016: Pyrgus alveus (Hübner, [1803]), Azerbaijan

Material: 10;, Nakhichevan, Ordubad, Agdere 23-24.07.2018; 10', Shemakha, Demirchi, 3-5.07.2017; 19, Yardimli, 17-

20.05.2016; 40"0", Shemakha, Archiman-Pirguli, near Shakhdag Milli Park,19-20.05.2013

18. Pyrgus jupei jupei(Alberti, 1967)

Devyatkin, 1994: Pyrgus jupei(Alberti, 1967), Nakhichevan, Zangezur (Zangəzur) Range (Bichenek) (Biçənək)

Hesselbarth, van Oorschot \& Wagener, 1995: Pyrgus jupei (Alberti, 1967) (=centralasiae Renner, 1991), Aserbeidschen

Devyatkin, 1997: Pyrgus jupei(Alberti, 1967), Nakhichevan, Zangezursky Mts., Bichenek Pass.

19. Pyrgus carlinae turcivola de Lattin, 1950

Tschikolovets, Nekrutenko, 2012: Pyrgus carlinae turcivola de Lattin, 1950, Gäncä (Gəncə) (Kirovobad), Dashkhasan (Daşkəsən)

Korb, Bolshakov, 2016: Pyrgus carlinae (Rambur, [1839]), Azerbaijan

Material: 10', Nakhichevan, Ordubad, Agdere 27.07.2018

20. Pyrgus cinarae cinarae (Rambur, [1839])

Effendi, 1970: Hesperia cinarae Rbr., Nakhichevan, Norashen (now Sharur (Şərur), Yaydja (Yaycı), Mt. Galin-gaya (Gəlingaya)

Tuzov, 1993: Pyrgus cinarae Rambur, 1839, Talysh; Armenian plateau

Devyatkin, 1997: Pyrgus cinarae (Rambur, [1839]), Nakhichevan Region, Zangezursky Mts., Bichenek Pass

Korb, Bolshakov, 2016: Pyrgus cinarae (Rambur, 1839), Azerbaijan

Material: 10', Lerik, Gosmalyan, 23.06.2017

21. Pyrgus carthami carthami (Hübner, [1813])

Effendi, 1970: Hesperia carthami Hb. var. moeschleri HS, Shemakha, Pirguli (Pirgulu); Ajikend

22. Pyrgus cacaliae cacaliae (Rambur, [1839]

Effendi, 1970: Hesperia cacaliae Rbr., Talysh, Orand, Pashagol (Paşagöl) Mt.

\section{Pyrgus sidae sidae (Esper, [1784])}

Effendi, 1970: Hesperia sidae Esp., Agdash - Garagan, Khaldan (Xaldan), Khanabad (Xanabad)

Korb, Bolshakov, 2016: Pyrgus sidae (Esper, [1782]), Azerbaijan

Material: 10`, Nakhichevan, Shakhbuz, Kyukyu, 27.07.2016; 5m, Shakhbuz, Nursu, 8.06.2017; 10", Ordubad, Agdere, 11.06.2017; 19, same place, 19.06.2019

24. Pyrgus malvae malvae (Linnaeus, 1758)

Effendi, 1970: Hesperia malvae L., everywhere from lowlands to highlands 
Effendi, 1970: Parnara nostrodamus Fabr., Kusari: Dustair, Bedirkala

Tschikolovets, Nekrutenko, 2012: Gegenes nostrodamus nostrodamus (Fabricius, 1793), Jelisavetpol (now Gandja)

Korb, Bolshakov, 2016: Gegenes nostrodamus (Fabricius, 1793), Azerbaijan

\section{Eogenes alcides alcides (Herrich-Schäffer, [1852])}

Romanoff, 1884: Hesperia Alcides HS, Ordoubad, Nakhitchevan

Jachontov, 1911: Eogenes (Parnara) alcides H.S., Aresh (now Khaldan (Xaldan)

Effendi, 1970: Parnara (Eogenes) alcides HS, Nakhichevan, Ordubad, Payiz, Unus; Djulfa (Culfa); Mingechaur (Mingəçevir)

Didmanidze, 1979: Eogenes alcides Herreich-Schäffer, 1852, Julfa, Ordubad

Didmanidze, 2004: Eogenes alcides Herreich-Schäffer, 1852, Julfa, Ordubad

Tschikolovets, Nekrutenko, 2012: Eogenes alcides alcides (Herrich-Schäffer, [1852]), Nakhichevan, Paraga-Tshaj

(Parağaçay)

Korb, Bolshakov, 2016: Eogenes alcides (Herich-Schäffer, [1852]), Azerbaijan

\section{Thymelicus sylvestris syriacus (Tutt, [1905])}

Romanoff, 1884: Hesperia Thaumas Hufn., Ordoubad; Talyche

Effendi, 1970: Adopaea (Thymelicus) thaumas Hufn., Baba-dag-Soygub, Erfi (ərfi), Ryuk (Rük); Kutkashen (now Gabala (Gəbələ), Laza; Semakha, Avakhil (Avaxıl)

Didmanidze, 1979: Thymelicus sylvestris Poda, 1761, road to Baku, 1700 m pass, lake surroundings; Nakhichevan,

Ordubad

Tuzov, 1993: Thymelicus sylvestris syriaca Tutt, 1905, Talysh; Armenian plateau

Didmanidze, 2004: Thymelicus sylvestris Poda, 1761, Baku district near - mountain Pass; Nakhichevan, Ordubad

Korb, Bolshakov, 2016: Thymelicus sylvestris (Poda, 1761), Azerbaijan

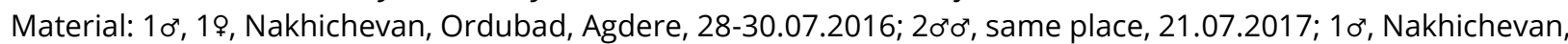

Shakhbuz, Bichenek, Shakhbuz National Park, 26.07.2016; 30', same place; 10", Nakhichevan, Shakhbuz, Kyukyu,

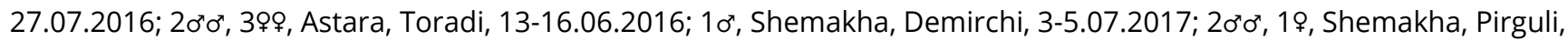

28.06.2013; 10, Siyazan, Beshbarmak, 4.06.2015

\section{Thymelicus lineola lineola (Ochsenheimer, 1808)}

Romanof, 1884: Hesperia Lineola O., Ordoubad

Christoph, 1886: Hesperia Lineola O., Talysh, Lenkoran

Effendi, 1970: Adopaea lineola O., everywhere in the meadows, in open areas

Didmanidze, 1979: Thymelicus lineola Ochsen., Ordubad; Khachmaz, Nizovaya

Tuzov, 1993: Thymelicus lineola lineola Ochsenheimer, 1808, Talysh; Armenian plateau

Didmanidze, 2004: Thymelicus lineola Ochsenheimer, 1808, Ordubad; Khachmaz, Nizovaya

Korb, Bolshakov, 2016: Thymelicus lineola (Ochsenheimer, [1808]), Azerbaijan

Material: $10^{\prime}$, Nakhichevan, Shakhbuz distr., between the village of Mahmudoba and Nursu, 30.05.2018; 20'0', Shemakha, Pyrguli, 25.06.2015

\section{Thymelicus hyrax hyrax (Lederer, 1861)}

Tuzov, 1993: Thymelicus hyrax Lederer, 1961, Talysh; Armenian plateau

Devyatkin, 1997: Thymelicus hyrax (Lederer, 1861), Nakhichevan Region, Daralagezsky Mts., Buzgov

Tschikolovets, Nekrutenko, 2012: Thymelicus hyrax hyrax (Lederer, 1861), Nakhichevan, Ordubad

Korb, Bolshakov, 2016: Thymelicus hyrax (Lederer, 1861), Azerbaijan

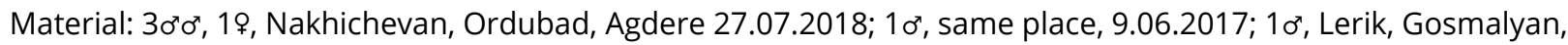
23.06.2017

\section{Thymelicus acteon acteon (Rottemburg, 1775)}

Effendi, 1970: Adopaea acteon Rott., Aterk, Yanshakh (Kelbadjar distr. (Kəlbəcər), Chaykend (Çaykənd) (Goygol distr. (Göygöl)

\section{Ochlodes sylvanus sylvanus (Esper, [1779])}

Kolenati, 1846: Hesperia Sylvanus Fabr., Karabagh

Romanoff, 1884: Hesperia Sylvanus Esp., Ordoubad; Hankynda (Xankəndi)

Effendi, 1970: Augiades sylvanus Esp., everywhere in the forests of the foothills and mountains

Didmanidze, 1979: Ochlodes venatus Brem. sbsp. hyrcana Chr., Turianchay Reservation; Khachmazç Nizovaya;

Nakhichevan: near Mount Shakhbus (Şaxbuz), Badamli (Badamlı)

Didmanidze, 2004: Ochlodes venatus Bremer et Grey, 1852, Turianchay Reservation; Khachmazç Nizovaya; Nakhichevan: near Mount Shakhbus, Badamli

Tschikolovets, Nekrutenko, 2012: Ochlodes sy/vanus sylvanus (Esper, [1779]), Nakhichevan, Kyukyu (Kükü) 
Korb, Bolshakov, 2016: Hesperia sy/vana (Esper, [1777]), Azerbaijan

Material: 10, 1\%, Kedabek, Novoivanovka - Novosaratovka vill., 19-21.06.2014; 10\%, 19, Shemakha, Demirchi, 24.06 .2015

32. Ochlodes hyrcana hyrcana (Christoph, 1893)

Ménétriés, 1832: Hesperia Sylvanus, Fab., Talyche Kolenati, 1846: Hesperia Sylvanus Fabr., Talysch

Ménétriés, 1855: Pamphila Sylvanus, Lenkoran

Christoph, 1874: Hesperia Sylvanus Esp., Lenkoran

Romanoff, 1884: Hesperia Sylvanus Esp., Lenkoran

Christoph, 1886: Hesperia Sy/vanus Esp., Lenkoran

Christoph, 1893: Hesperia Hyrcana Chr., Lenkoran

Heyne, [1895]: Pamphila sylvanus Esp., Lenkoran

Heyne, [1895]: Thym.Hyrcana Chr., Lenkoran

Evans, 1949: Ochlodes venata Sub.-sp. hyrcana Christoph, 1893, Lenkoran

Häuser, 1982: Ochlodes hyrcanus (Christoph, 1893), Lenkoran

Tuzov, 1993: Ochlodes hyrcana hyrcana Christoph, 1893, Talysh

Devyatkin, 1997: Ochlodes hyrcana hyrcana Christoph, 1893, Talysh Mts.

Tschikolovets, Nekrutenko, 2012: Ochlodes hyrcana hyrcana (Christoph, 1893), Masally (Masallı); Talysh, Lerik; Shuvi vill. (Şuvi)

Korb, Bolshakov, 2016: Hesperia hyrcana Christoph, 1893, Azerbaijan

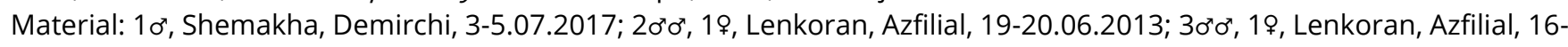
19.09.2013; 20'0', same place, 25-28.05.2013

\section{Hesperia comma comma (Linnaeus, 1758)}

Effendi, 1970: Augiades (Erinnis) comma L., everywhere

Tuzov, 1993: Hesperia comma comma Linnaeus, 1758, Talysh

Korb, Bolshakov, 2016: Hesperia comma (Linnaeus, 1758), Azerbaijan

34. Hesperia comma pallida (Staudinger, 1901)

Ménétriés, 1855: Pamphila Comma, Lenkoran

Tschikolovets, Nekrutenko, 2012: Hesperia comma comma (Linnaeus, 1758), Nakichevan, Ordubad distr., Pazmara, NyusNyus; Zangezur Mts., Bitshenek; Talysh, Kosmolyan, Hili-Dara

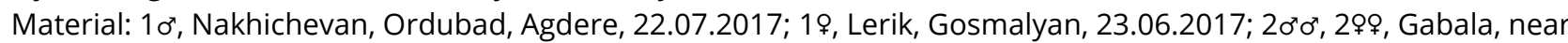
Vendam and Bunud vill., 5-8.07.2016

35. Carterocephalus palaemon palaemon (Pallas, 1771)

Effendi, 1970: Pamphila palaemon Pall., Evlakh (Evlax), Gazakh (Gazax), Khachmaz (Xaçmaz), Shemakha, Kirovka

36. Carterocephalus silvicolus silvicolus (Meigen, 1830)

Effendi, 1970: Pamphila silvius Knoch., Shemakha, Pirkuli; Gey-Gol (Göygöl) Lake; Astara-Sarak

37. Heteropterus morpheus morpheus (Pallas, 1771)

Effendi, 1970: Heteropterus morpheus Pall., Khachmaz, Kusarchay (Kusarçay)

38. Papilio alexanor orientalis Romanoff, 1884

Family Papilionidae Latrielle, [1802])

Romanoff, 1884: Papilio Alexanor Esp. var. Orientalis Rom., Ordoubad

Heyne, [1895]: [Papilio Alexanor Esp.] var. orientalis Romanoff, Ordubad

Effendi, 1970: P.alexanor Esp. ssp. orientalis Rom., Djuga, Ordubad

Didmanidze, 1979: Papilio alexanor Esp. (orientalis Rom.), Nakhichevan, Ordubad

Bollino \& Sala, 2004: Papilio alexanor orientalis Romanoff, 1884, Mt.Daralages, Mt.Zangezur, env. D”Ordoubad

Tschikolovets, Nekrutenko, 2012: Papilio alexanor orientalis Romanoff, 1884, Nakhichecan, Daralagezsky Mts., Buzgov

Korb, Bolshakov, 2016: Papilio alexanor orientalis Romanoff, 1884, Ordoubad

Material: 10', Nakhichevan, Ordubad, Agdere, 15-17.06.2019

39. Papilio machaon machaon Linnaeus, 1758

Ménétriés, 1832: Papilio Machaon, Linn., Lenkoran

Christof, 1886: Papilio Machaon L., Lenkoran

Jachontov, 1911: Papilio machaon L., Ordubad, Aresh (now Khaldan)

Effendi, 1970: Papilio machaon L. ssp. sphyrus Hb., throughout the lowlands and in the foothills

Didmanidze, 1979: Papilio machaon L., Turianchay Reservation; Nakhichevan, Ordubad 
Tuzov, 1993: Papilio machaon syriacus Eller, 1939, Talysh; Armenian plateau

Kaabak, Tarasov \& Tuzov, 1997: Papilio machaon ssp. syriacus Eller, 1939, Talysh Mts.

Tschikolovets, 2003: Papilio machaon, Alty-Agatsh

Tschikolovets, Nekrutenko, 2012: Papilio machaon machaon Linnaeus, 1758, Gobustan

Korb, Bolshakov, 2016: Papilio machaon Linnaeus, 1758, Azerbaijan

Material: 10', Nakhichevan, Ordubad, Agdere, 15-17.06.2019

40. Papilio demoleus demoleus Linnaeus, 1758

Material: 1 m, Lenkoran, Azfilial settlement, 25-28.05.2013. Indicated for the first time for the fauna of the Caucasus.

41. Iphiclides podalirius podalirius (Linnaeus, 1758)

Ménétriés, 1855: Papilio Podalirius, Nachitshewan

Ménétriés, 1859: Papilio Podalirius Lin., Nachitschewan

Christoph, 1874: Papilio Podalirius L., Lankoran

Christof, 1886: Papilio Podalirius, Lenkoran

Effendi, 1970: Papilio podalarius L., ssp. armeniaca B-H., everywhere in the foothills, rarely in the mountains; Astara

Didmanidze, 1979: Iphiclides podalirius L., Nakhichevan, river Paragachai gorge; Mount Kapujukh; river Arax gorge near

Ordubad, Kilit

Tuzov, 1993: Iphiclides podalirius persica Verity, 1911, Talysh; Armenian plateau

Kaabak, Tarasov \& Tuzov, 1997: Iphiclidis podalirius ssp. persica Verity, 1911, Talysh; Armenian Highland

Didmanidze, 2004: Iphiclides podalirius Linnaeus, 1758, Nakhichevan, river Paragachai gorge; Mount Kapujukh; river

Arax gorge near Ordubad, Kilit

Tschikolovets, Nekrutenko, 2012: Iphiclides podalirius (Linnaeus, 1758), Latshinsky (Laçın) distr., Kyurdgadzhi (Kürhacı)

vill.; Nakhichevan, Bilav

Korb, Bolshakov, 2016: Iphiclides podalirius (Linnaeus, 1758), Azerbaijan

Material: 10", Astara, Toradi, 13-16.06.2016; 19, Belokani, Beshbulag, Djidjikhana, 25.06.2013; 10", Nakhichevan, Ordubad,

Agdere, 15-17.06.2019; 10", Kuba, Tengealti, 9.07.2019

42. Zerynthia polyxena polyxena ([Denis \& Schiffermüller], 1775)

Effendi, 1970: Thais (Zerynthya) polyxena Sehiff., Zakataly, Djari (Carı); Kutkashen (now Gabala), Bum; Shemakha, Pirguli; Adjikend; Goy-Gol Lake; Lachin, Yanshakh; Talysh, Yardimly (Yardımlı)

43. Allancastria caucasica caucasica (Lederer, 1864)

Effendi, 1970: Thais (Zerynthya) cerisyi God. ssp. caucasica Ld., Belokany, Akh-Kimal

Tschikolovets, Nekrutenko, 2012: Zerynthia polyxena ([Denis \& Schiffermüller], 1775), Zakataly (Zagatala) distr., Parzivan (Pərzivan)

Korb, Bolshakov, 2016: Allancastria caucasica (Lederer, 1864), Azerbaijan

44. Driopa mnemosyne mnemosyne (Linnaeus, 1758) -

Romanoff, 1884: Parnassius Mnemosyne L. ver. Nubilosus Chr., Ordoubad

Bryk \& Eisner, 1932: Parnassius Mnemosyne subsp. delgranprincipe Bryk., Jelizavethpol (now Gandja)

Effendi, 1970: Parnassius mnemosyne L., everywhere in the forest areas of the foothills and mountains of the Greater and Lesser Caucasus and Talysh

Tuzov, 1993: Parnassius Mnemosyne nubilosus Christoph, 1873, Talysh; Armenian plateau

J.-C.Weiss, 1999: Parnassius Mnemosyne ssp. daghestanus Bryk \& Eisner, 1934, northern Azerbaijan

S.K.Korb, 2005: Driopa Mnemosyne taleschensis Ebert, 1975, Talysh

Tschikolovets, Nekrutenko, 2012: Parnassius mnemosyne mnemosyne (Linnaeus, 1758), Nakhichevan, Daralagez Mts., Buzgov; Khurs (Xurs)

Korb, Bolshakov, 2016: Driopa mnemosyne (Linnaeus, 1758), Azerbaijan

45. Driopa nordmanni nordmanni Ménétriés in Siemaschko, [1850]

Effendi, 1970: Parnassius nordmanni Mén., southern and eastern slopes of Babadag, Shahdag, Bazarduzi mountains

46. Driopa phoebus phoebus (Fabricius, 1793)

Effendi, 1970: Parnassius delius Esp., between the peaks Shakhdag and Bazar-Duzi.

The author writes that he caught the only one specimen on the southeastern slopes of the Shahdag-Bazarduzi system in July. Unfortunately, this specimen was not preserved in the collection, but there is a photo in the dissertation confirming that this specimen existed and it is really Parnassius phoebus. 
Effendi, 1970: Parnassius apollo L. ssp. caucasicus Pag., Shemakha: Muganli (Muğanlı), Angekharan (əngəxaran), Pirguli, Avakhil, Girkh-bulag (Gırxbulağ); Goy-Gol Lake; Lachin, Yanshakh; Kelbajar, Istisu, Kafan

Turlin \& Manil, 2005: Parnassius (Parnassius) apollo Linne, 1758, Azerbaijan

Tschikolovets, Nekrutenko, 2012: Parnassius apollo suaneticus Arnold, 1909: Turianchaysky nature reserve

Korb, Bolshakov, 2016: Parnassius apollo kashtshenkoi Sheljuzhko, 1908, Transcaucasus, Azerbaijan

48. Leptidea sinapis sinapis (Linnaeus, 1758)

\section{Family Pieridae Duponchel, [1835])}

Effendi, 1970: Leptidia sinapis L., everywhere in the meadows, lowlands and foothills

Didmanidze, 1979: Leptidia sinapis L., Turianchay Reservation

Didmanidze, 2004: Leptidea sinapis Linnaeus, 1758, Turianchay Reservation

Tschikolovets, Nekrutenko, 2012: Leptidea sinapis sinapis (Linnaeus, 1758), Nakhichevan, Ordubad

Korb, Bolshakov, 2016: Leptidea sinapis (Linnaeus, 1758), Azerbaijan

Material: 10", Lenkoran, Azfilial, 19-20.06.2013; 10", Zakatala, from kombinat to State Reserve, 24.06.2014; 19, Siyazan, Beshbarmak, 4.06.2015; 19, Lenkoran, Azfilial, 16-19.09.2013; 5m, 29\%, Kuba, Afurdja, 8.07,2019; 10', Nakhichevan,

Ordubad, Agdere, 11.06.2017

\section{Leptidea duponcheli lorkovici Pfeiffer, [1932]}

Romanoff, 1884: Leucophasia Duponcheli Srgr., Ordoubad

Effendi, 1970: Leptidea duponcheli Stgr., Ordubad; Talish, Zuvand

Didmanidze, 1979: Leptidea duponcheli Stgr., Turianchay Reservation; Khachmaz, Nizovaya; Nakhichevan, Ordubad vic.

Tuzov, 1997: Leptidea dupocheli ssp. Iorkovici (Pfeiffer, 1932), Nakhichevan Region, Daralagezsky Mts., Buzgov

Tuzov, 1997: Leptidea duponchelissp.vartiani Gross et Ebert, 1975, Talysh

Tschikolovets, 2003: Leptidea duponcheli lorkovici, Alty-Agatsh

Tschikolovets, Nekrutenko, 2012: Leptidea duponcheli lorkovici Pfeiffer, [1932]), Nakhichevan, val.fl.Paragatshai Korb, Bolshakov, 2016: Leptidea duponcheli (Staudinger, 1871), Azerbaijan

Material: $10^{\prime \prime}$, Nakhichevan, Ordubad, Agdere, 28-30.07.2016; 10", Shakhbuz, Nursu, 8.06.2017; 20'0", Nakhichevan,

Shakhbuz distr., between the village of Mahmudoba and Nursu, 30.05.2018

50. Anthocharis cardamines cardamines (Linnaeus, 1758)

Effendi, 1970: Euchloe cardamines L.ab.turritis O., everywhere in the foothills and mountains, to the upper boundary of the forest

Didmanidze, 1979: Anthocharis cardamines L., Turianchay Reservation; Nakhichevan, Paragachay River Gorge, m. Kapudzhuh

Tuzov, 1993: Anthocharis cardamines cardamines Linnaeus, 1785, Talysh; Armenian plateau

Tuzov, 1997: Anthocharis cardamines ssp. cardamines (Linnaeus, 1785), Talysh; Armenian Highland

Tschikolovets, Nekrutenko, 2012: Anthocharis cardamines cardamines (Linnaeus, 1758). Nakhichevan, Daralagezsky Mts., Buzgov

Korb, Bolshakov, 2016: Anthocharis cardamines (Linnaeus, 1758), Azerbaijan

Material: 3ơơ 19, Siyazan, Galaalty, 18.05.2017

\section{Anthocharis gruneri armeniaca Christoph, 1893}

Romanoff, 1884: Anthocharis Gruneri HS, Ordoubad

Christoph, 1893: Anthocharis Gruneri HS. ver. armeniaca, Ordubad

Heyne, [1895]: Anthocharis Gruneri H.S., ver. armeniaca Christoph, Ordubad

Effendi, 1970: Euchloe gruneriHS ssp. armeniaca Chr., everywhere in the foothills and mountains of the Nakhichevan AR Tuzov, 1993: Anthocharis gruneri armeniaca Christoph, 1893, Talysh; Armenian plateau

Tuzov, 1997: Anthocharis grunerissp. armeniaca Christoph, 1893, Talysh Mts.; Armenian Highland

Tomonaga, 1998: Anthocharis grunerissp. armeniaca Christoph, 1893, Aserbaidschan

Tschikolovets, 2003: Anthocharis gruneri, Talysh, Zuvand; Nakhichevan, Buzgov

Tschikolovets, Nekrutenko, 2012: Anthocharis gruneri armeniaca Christoph, 1893, Nakhichevan, Daralagez Mts., Buzgov; Talysh, Gosmolyan

Korb, Bolshakov, 2016: Anthocharis gruneri Herrich-Schäffer, 1845, Azerbaijan

\section{Anthocharis damone eunomia Freyer, ([1851])}

Alphéraky, 1910: Anthocharis damone B., Ordubad

Effendi, 1970: Euchloe damone B., Ordubad, Kilit

Didmanidze, 1979: Anthocharis damone Bsd., Nakhichevan, Ordubad

Korshunov, 1972: Anthocharis damone Boisduval, 1836, Ordubad 
53. Zegris eupheme menestho (Ménétriés, 1832)

Ménétriés, 1832: Pieris Menestho: Zouvant sur les montagnes de Talyche

Ménétriés, 1855: Zegris Menestho, Lenkoran

Lederer, 1870: Zegris Eupheme Esp. var. Menestho Mén., Talyche

Hemming, 1929: Zegris eupheme ssp. menestho, Ménétriés, Ordubad, Baku

Effendi, 1970: Zegris eupheme Esp. ssp. menestho Men., the slopes of the Bozdag (Bozdağ) hills system, in the region of Geokchai (Gökçay) and in the region of Khaldan; Nakhichevan, Ordubad; Talysh, Zuvand

Didmanidze, 1974: Zegris eupheme Esp., Mashtaga (Maştağa) neighborhood (Absheron); surroundings - lake Sarysu (Sarısu); Pirkuli Nature Reserve, the Dybrarar (Dübrar) Range along the gorge of the Pirsagat River; Nakhichevan, Ordubad Nekrutenko, 1990: Zegris eupheme ssp. menestho (Mén.), Kura and Arax valleys; Talysh Mts.

Tuzov, 1993: Zegris eupheme eupheme Esper, 1805, Talysh; Armenian plateau

Tuzov, 1997: Zegris eupheme eupheme (Esper, [1804]) (= menestho Ménétriés, 1832), Talysh; Armenian Highland

Tuzov, 1997: Zegris eupheme menestho Ménétriés, 1832, Talysh Mts., Lerik Distr., Gosmolyan

Didmanidze, 2004: Zegris eupheme Esper, 1805: Pirkuli Reservation; near Lake Sari-su, Mashtaga, river Pirsagat gorge near Diubrar mount.

Didmanidze, 2004: Zegris menestho Ménétriés, 1832: Eldari and Shirvani (Şirvan) steppe; Nakhichevan, Bilyav and Ordubad

S.K.Korb, 2005: Zegris eupheme menestho Ménétriés, 1832, region between Kura and Arax rivers

Tschikolovets, Nekrutenko, 2012: Talysh, Lerik, Gosmolyan; Jelizavetpol (now Gandja)

Korb, Bolshakov, 2016: Zegris eupheme (Esper, [1805]), Azerbaijan

54. Euchloe ausonia taurica Röber, [1907]

Romanoff, 1884: Anthocharis Belia Cr., Ordoubad

Romanoff, 1884: Anthocharis Belia Cr. var. Ausonia Hb, Ordoubad, Talyche

Christoph, 1886: Anthocharis Belia Cr., Lenkoran

Effendi, 1970: Euchloe (Anthocharus) belia Chr., everywhere in the foothills

Didmanidze, 1979: Euchloe ausonia Hubn., (= belia Stoll.), road to Baku; Nakhichevan, Ordubad

Tuzov, 1993: Euchloe ausonia volgensis Krulikowsky, 1897, Talysh; Armenian plateau

Tuzov, 1997: Euchloe ausonia ssp. volgensis Krulikowsky, 1897, Talysh; Armenian Highland

Didmanidze, 2004: Euchloe ausorina Hübner, 1804, Baku district

Back, Knebelsberger \& Miller, 2008: Euchloe ausonia taurica, Talysh

Tschikolovets, Nekrutenko, 2012: Euchloe ausonia taurica Röber, [1907], Elisavetpol (now Gandja); Nakhichevan,

Nehram, Daralgez (Daralgöz) Mts., Buzgov

Korb, Bolshakov, 2016: Euchloe ausonia (Hübner, [1803]), Azerbaijan

55. Euchloe crameri sovinskyi Sheljuzhko, 1928

Tshikolovets, 2011: Euchloe crameri sovinskyi Sheljuzhko, 1928, Coastal areas of Caspian Sea from N.Azerbaijan to N. - W. Kazakhstan

Tschikolovets, Nekrutenko, 2012: Euchloe crameri sovinskyi Sheljuzhko, 1928, Baku

56. Pieris rapae rapae (Linnaeus, 1758)

Ménétriés, 1832: Pieris rapae, Linn., Lenkoran

Christof, 1886: Pieris rapae L., Lenkoran

Bollow, 1930: Pieris rapae subsp. transcaucasica Std., Gebirgen um Jelisavetpol

Effendi, 1970: Pieris rapae L., everywhere from lowland to subalpine zone

Didmanidze, 1979: Pieris rapae L., road to Baku; Turianchay Reserve; Mil steppe, lake Sarysu; Gobustan

Tuzov, 1997: Pieris rapae ssp. meleager(Hemming, 1934), Talysh

Korb, Bolshakov, 2016: Pieris rapae (Linnaeus, 1758), Azerbaijan

Material: 1 m, Nakhichevan, Ordubad, Agdere, 28-30.07.2016; 10, Astara, Toradi, 13-16.06.2016; 10", 19, Kedabek, Novoivanovka -Novosaratovka vill., 19-21.06.2014; 3\%o, Lenkoran, Azfilial, 25-28.05.2013

\section{Pieris ergane detersa Verity, [1908]}

Jachontov, 1911: Pieria ergane H.G., Ordubad; Aresh

Effendi, 1970: Pieris ergane H.-G., Нахичевань, Ордубад; Талыш, Зуванд

Didmanidze, 1979: Pieris ergane H-G., surroundings of the Mingechaur reservoir; Nakhichevan, mt. Kaputzhuh

Nekrutenko, 1990: Pieris (Artogeia) ergane (Greyer in Hb.) Talysh

Tuzov, 1997: Pieris ergane ssp. detersa Verity, 1911, Nakhichevan Region, Daralagezsky Mts., Buzgov; Zangezursky Mts., Nyusnyus

Tuzov, 1997: Pieris ergane ssp. elbursina Bytinski-Salz, 1937, Talysh

Didmanidze, 2004: Pieris ergane Geyer, 1828, lori river near Resrvoir Mingechauri

S.K.Korb, 2005: Artogeia elbursina elbursina Bytinski-Salz, 1937, Talysh 
Tschikolovets, Nekrutenko, 2012: Pieris ergane detersa Verity, [1908], Nakhichevan, Pazamara; Buzgov Korb, Bolshakov, 2016: Pieris ergane (Geyer, [1827 - 1828]), Azerbaijan

Material: $1 \sigma^{x}$, Nakhichevan, Ordubad, Agdere, 21.07.2017; 1\%, Lerik, Gosmalyan, 21-22.06.2017

58. Pieris napi meridionalis Heyne, 1895

Lederer, 1864: Pieris napi L., Helenendorf (now Goygel (Göygol) city) bei Elizabethpol

Christof, 1874: Pieria Napi L., Lenkoran

Christof, 1886: Pieria napi L., Lenkoran

Verity, 1906: Pieris napi, L. forme suffuse, Verity, Jelizavetpol

Effendi, 1970: Pieris napi L., throughout the lowlands and mountains

Didmanidze, 1979: Pieris napi sbsp. caucasica Vsty., Mil steppe, Lake Sarysu; Lake Aggel; Nakhichevan, Paragachay river gorge, Kaputzhuh range; Shahbuz

Eitschberger, [1984]: Pieris pseudorapae suffuse Sheljuzko, 1931, Alexeevca (now Burdjali (Bürcəli) Lenkoranskij distr., Lenkoran

Tuzov, 1993: Artogeia pseudorapae suffuse Sheljuzhko, 1931, Talysh; Armenian plateau

Tuzov, 1997: Pieris pseudorapae ssp. suffusa Sheljizhko, 1931 (= postsuffusa Eitschberger, 1983), Talysh; Armenian

Highland

Tschikolovets, Nekrutenko, 2012: Pieris napi meridionalis Heyne, 1895, Nakhichevan, Bitshenek

Korb, Bolshakov, 2016: Pieris napi (Linnaeus, 1758), Azerbaijan

Material: 10', 1\%, Nakhichevan, Shakhbuz, Kyukyu, 27.07.2016; 19, Nakhichevan, Ordubad, Agdere, 9-12.06.2017; 200", Lenkoran, Azfilial,1-4.10.2012; 10; 19, same place, 25-28.05.2013; 10;, Shemakha, Archiman-Pirguli, near Shakhdag Milli Park,19-20.05.2013; 10", 19, Zakatala, Djidjikhana, 10-12.07.2015; 10", Apsheron, near Zoologican Institute, 10.05.2013; 10", Ismailly Kaladjik, Velasin, 21-23.07.2014; 3\%, Kuba, Afurdja, 8.07,2019

59. Pieris krueperi krueperi Staudinger, 1860

Romanoff, 1884: Pieris Krueperi Stgr., Ordoubad

Effendi, 1970: Pieris krueperi Stgr., Talish, Gosmolyan; Nakhichevan, Ordubad, Unus, Paiz

Didmanidze, 1979: Pieris krueperi Stgr., Nakhichevan, Ordubad vic.

Tuzov, 1993: Artogeia krueperi krueperi Staudinger, 1860, Talysh; Armenian plateau

Tuzov, 1997: Pieria krueperi krueperi Staudinger, 1860, Talysh; Nakhichevan Region, Ordubad; Zangezursky Mts., Nyusnyus

Didmanidze, 2004: Pieris krueperi Staudinger, 1860, Nakhichevan, Ordubad

Korb, Bolshakov, 2016: Pieris krueperiStaudinger, 1860, Azerbaijan

60. Pieris brassicae brassicae (Linnaeus, 1758)

Ménétriés, 1859: Pieris Brassicae Lin., Nachitschewan

Becker, 1873: Pieris Brassicae, Baku

Christoph, 1877: Pieris brassicae L., Lenkoran

Christoph, 1886: Pieris brassicae L., Lenkoran

Jachontov, 1911: Pieris brassicae L., Lenkoran

Effendi, 1970: Pieris brassicae L., everywhere except for the alpine belt

Didmanidze, 1979: Pieris rapae L., road to Baku, Turianchay Reserve; Mil steppe, Lake Sarysu environs; Gobustan

Tuzov, 1993: Pieris brassicae brassicae Linnaeus, 1758, Talysh

Tuzov, 1997: Pieris brassicae brassicae (Linnaeus, 1758), Talysh

Tschikolovets, Nekrutenko, 2012: Pieris brassicae brassicae (Linnaeus, 1758), Terter (Tərtər) (gub.Elisavetpol)

Korb, Bolshakov, 2016: Pieris brassicae (Linnaeus, 1758), Azerbaijan

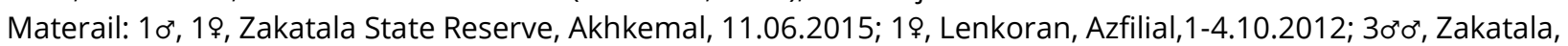

Djidjikhana, 10-12.07.2015; 10', Lenkoran, Azfilial, 16-19.09.2013; 19, same place, 6-9.11.2012; 19, Ismailly Kaladjik, Velasin,

21-23.07.2014; 1\%, Massali, near Kubin and Mijankyu vill., 5.05.2014

\section{Pontia chloridice chloridice (Hübner, [1813])}

Romanoff, 1884: Pieris Chloridice Hb., Ordoubad

Effendi, 1970: Leucochloe.chloridice Hb., everywhere in the lowlands, in the foothills and mountains to the upper boundary of the forest; in Talysh with the main form flies ssp. albidice Stgr. - Talysh, Sarak

Didmanidze, 1979: Pontia chloridice Hübn., Nakhichevan, Ordubad

Tuzov, 1993: Pontia chloridice chloridice Ochsenheimer, 1816, Talysh; Armenian plateau

Tuzov, 1997: Pontia chloridice chloridice (Hübner, 1808), Talysh; Armenian Highland

Didmanidze, 2004: Pontia chloridice Hübner, [1808-1813], Nakhichevan, Ordubad

Korb, Bolshakov, 2016: Pontia chloridice (Hübner, [1813]), Azerbaijan 
62. Pontia daplidice daplidice (Linnaeus, 1758)

Ménétriés, 1832: Pieris daplidice, Linn., Lenkoran

Ménétriés, 1855: Pieris Daplidice, Lenkoran

Becker, 1873: Anthocharis var. Bellidice, Lenkoran

Christoph, 1874: Pieris Daplidice L., Lenkoran

Christoph, 1877: Pieris Daplidice L., Baku

Christoph, 1886: Pieris Daplidice L., Lenkoran

Stauder, 1924: Leucochloe daplidice L., Terter, Gouv.Jelizavetpol

Effendi, 1970: Leucochloe daplidice L., throughout the lowlands, in the foothills and mountains, to the upper boundary of the forest

Didmanidze, 1979: Pontia daplidice L., Turianchay Reserve; Nakhichevan, road to Ordubad; Julfa, geyser

Tuzov, 1993: Pontia daplidice daplidice Linnaeus, 1758, Talysh; Armenian plateau

Tuzov, 1997: Pontia daplidice daplidice (Linnaeus, 1758), Talysh; Armenian Highland

Didmanidze, 2004: Pontia daplidice daplidice Linnaeus, 1758, Steppe Mili, near Lake Ag-giol (Ağgöl); Saatli (Saatlı),

Jdanovsk (now Beylagan (Beyləqan); Gobustan, Mount Turogai

Tschikolovets, Nekrutenko, 2012: Pontia daplidice daplidice (Linnaeus, 1758), Nakhichevan, Ordubad

Korb, Bolshakov, 2016: Pontia daplidice (Linnaeus, 1758), Azerbaijan

Material: 10;, Zakatala State Reserve, Akhkemal, 11.06.2015; 19, Kedabek, Novoivanovka -Novosaratovka vill., 19-

21.06.2014; 20 $0^{\circ}$, Zakatala, Djidjikhana, 10-12.07.2015; 10', Lenkoran, Azfilial, 6-9.11.2012; 10', Kuba, Afurdja 9.07.2019

\section{Pontia callidice chysidice (Herrich-Schäffer, [1844])}

Effendi, 1970: Leucochloe callidice Esp., southern and eastern slopes of the Greater Caucasus from Belokani to

Shemakha

Didmanidze, 1979: Pontia callidice Esp., Karabakh (Garabağ) steppe, Murovdag (Mrovdağ) ridge

Tuzov, 1993: Pontia callidice chysidice Herrich-Schäffer, 1843, Talysh

Tuzov, 1997: Pontia callidice chysidice (Herrich-Schäffer, 1843), Talysh

Didmanidze, 2004: Synchloe callidice Hübner, [1799-1800], Karabakh

Korb, Bolshakov, 2016: Pontia callidice (Hübner, [1800]), Azerbaijan

64. Aporia crataegi crataegi (Linnaeus, 1758)

Ménétriés, 1832: Pieris crataegi, Linn., Talyche

Lederer, 1870: Pieris Crataegi L., Talyche

Christoph, 1886: Aporia Crataegi L., Lenkoran

Jachontov, 1911: Aporia crataegi L., Lenkoran, Arus village

Effendi, 1970: Aporia crataegi L., everywhere in the foothills and mountains, to the upper boundary of the forest

Didmanidze, 1979: Aporia crataegi L., road to Baku, environs of the pass; Nakhichevan, Paragachay valley, Bilav

Tuzov, 1993: Aporia crataegi iranica Forster, 1939, Talysh; Armenian plateau

Tuzov, 1997: Aporia crataegissp. iranica Forster, 1939, Talysh Mts.; Armenian Highland

Della Bruna, Gallo \& Sbordoni, 2004: Aporia crataegi crataegi Linnaeus, 1758, Talysh Mts.

Korb, Bolshakov, 2016: Aporia crataegi (Linnaeus, 1758), Azerbaijan

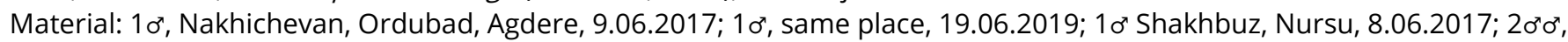
Astara,Toradi, 13-16.06.2016; 10', 19, Shemakha, Archiman-Pirguli, near Shakhdag Milli Park,19-20.05.2013

65. Colias erate erate (Esper, [1805])

Effendi, 1970: Colias erate Esp., everywhere

Didmanidze, 1979: Colias erate Esp., Mil steppe, Lake Aggel; Nakhichevan, Ordubad, Julfa, geyser

Tuzov, 1993: Colias erate erate (Esper, 1805), Talysh; Armenian plateau

Didmanidze, 2004: Colias erate erate Esper, 1808, Millis steppe, near Lake Ag-Giol; Nakhichevan, Ordubad, Julfa

Korb, Bolshakov, 2016: Colias erate (Esper, [1801]), Azerbaijan

Material: 10", 19, Lenkoran, Azfilial,1-4.10.2012

66. Colias alfacariensis alfacariensis Ribbe, 1905

Romanoff, 1884: Colias Hyale L. ab. Sareptensis Stgr., Ordoubad

Christoph, 1886: Colias Hyale L., Lenkoran

Effendi, 1970: Colias hyale L. ssp. meridionalis Krul., throughout the open forestless foothills, less frequent in lowlands and mountains

Didmanidze, 2004: Colias hyale L., surroundings of Baku; Nakhichevan, Ordubad, Paragachay river gorge, Bilav

Reissinger, 1989: Colias alfacariensis fontanei, gen. III (aut.) postfontainei, Nachicevan

Reissinger, 1989: Colias alfacariensis fontanei, gen. fontainei, Szemacha Distr., Kirowka

Reissinger, 1989: Colias alfacariensis fontanei, gen. IV ultimafontainei, Nachycevan

Reissinger, 1989: Colias alfacariensis fontanei, Reissinger, 1989, Nakhitschewan 
Tuzov, 1993: Colias alfacariensis fontanei, Reissinger, 1989, Talysh; Armenian plateau

Tuzov, 1997: Colias alfacariensis ssp. fontanei, Reissinger, 1989, Talysh; Armenian Highland

Didmanidze, 2004: Colias hyale Linnaeus, 1758, near Baku; Nakhichevan, Ordubad

Tschikolovets, Nekrutenko, 2012: Colias alfacariensis alfacariensis Ribbe, 1905, Adzhi-Kent

Korb, Bolshakov, 2016: Colias alfacariensis Ribbe, 1905, Azerbaijan

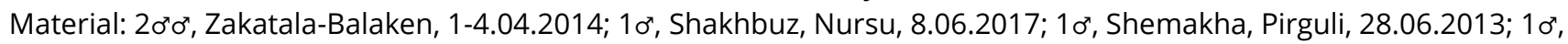
Belokani, Beshbulag, Djidjikhana, 25.06.2013; Massali, near Kubin and Mijankyu vill., 5.05.2014; 10, 2\%क, Kuba, Afurdja 9.07.2019

67. Colias croceus croceus (Geoffroy in Fourcroy, 1785)

Lederer, 1864: Colias edusa Fab., Helenendorf bei Elizavetpol

Becker, 1873: Colias Edusa F., Lenkoran

Christoph, 1874: Colias Edusa F., Lenkoran

Christoph, 1886: Colias Edusa F., Lenkoran

Effendi, 1970: Colias croceus Fourcz. (= edusa F.), throughout the subalpine belt

Didmanidze, 1979: Colias croceus F., Turianchay Reserve; road to Baku; Mil steppe, Saatly; the neighborhood of

Zhdanovsk; ridge Turagay; Nakhichevan, Ordubad surr., border checkpoint; Julfa, geyser; Bilav; Paragachay Gorge; road to

Ordubad; ridge Shahbuz

Tuzov, 1993: Colias crocea crocea Geoffroy, 1785, Talysh; Armenian plateau

Verhulst, 2000: Colias crocea Fourcroy, 1785, Azerbeijan

Tschikolovets, Nekrutenko, 2012: Colias croceus croceus (Geoffroy in Fourcroy, 1785), Nakhichevan, Ordubad, Alakhi vill.;

Talysh, Lerik: Nakhichevan, Bilav, Bitshenek; Shuvi (Şuvi) vill.; gub.Elizavetpol, Terter

Korb, Bolshakov, 2016: Colias crocea (Geoffroy, 1785), Azerbaijan

Material: 10", Nakhichevan, Badamli, 27.07.2016; 2\%9, Nakhichevan, Ordubad, Agdere, 28-30.07.2016; 10', same place, 28.07.2016; $10^{\prime \prime}$, same place, 24.07.2017; 10", 19, same place, 9.06.2017; 20'0", Nakhichevan, Shakhbuz, Bichenek, Shakhbuz National Park, 26.07.2016; 10', Nakhichevan, Shakhbuz, Kyukyu, 27.07.2016; 200', 19, Zakatala State Reserve, Akhkemal, 11.06.2015; 10", Shemakha, Demirchi, 3-5.07.2017, 10", Yardimli,17-20.05.2016; 10', 19, Kedabek, Novoivanovka Novosaratovka vill., 19-21.06.2014; 10 , 19, Astara,Toradi, 13-16.06.2016; 10', Zakatala, from kombinat to State Reserve,

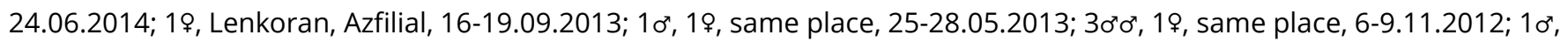

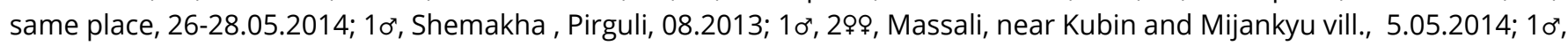
Kuba, Afurdja, 8.07,2019

68. Colias myrmidone myrmidone (Esper, [1781])

Effendi, 1970: Colias myrmydone Esp. ssp. caucasica Stgr., Shemakha, Pirkuli; Kutkashen (now Gabala), Laza Korb, Bolshakov, 2016: Colias myrmidone (Esper, [1781]), Azerbaijan

69. Colias caucasica caucasica Staudinger, 1870

Effendi, 1970: Colias caucasica Stgr. (= olga Rom.), Elenedorf (Khanlar, now Goygol); Guba distr., Gonakhkend, Ryuk; southern slopes of Shah-Dag

Didmanidze, 1979: Colias caucasica Stgr., Nakhichevan, Ordubad, Paragachay river gorge

Verhulst, 2000: Colias caucasica caucasica Staudinger, 1871, Helenendorf, Zangezur distr., Elizavetpol, Ordubad

Didmanidze, 2004: Colias caucasica Staudinger, 1871, Paragachay gorge near state border, Ordubad

Korb, Bolshakov, 2016: Colias caucasica Staudinger, 1871, Azerbaijan

70. Colias aurorina aurorina Herrich- Schäffer, [1850]

Lederer, 1864: Colias aurorina H.- Sch., Helenendorf bei Elisabethpol

Romanoff, 1884: Colias Aurorina HS, Zangezour, Elisabethpol

Nekrutenko, 1990: Colias aurorina H. - S., Talysh Mts.; Dzhavakhet-Armenian plateau)

Effendi, 1970: Colias aurorina HS (= tamara Nordm.), Gonakhkend, Ryuk; Kyapaz Mt. (Kəpəz); Nakhichevan, Paiz

Tuzov, 1993: Colias aurorina aurorina Herrich- Schäffer, 1850, Armenian plateau; Talysh

Verhulst, 1994: Colias aurorina aurorina Herrich- Schäffer, 1850, Azerbaidjan

Tuzov, 1997: Colias aurorina aurorina H errich- Schäffer, [1850], Nakhichevan region, Zangezursky Mts., Bichenek Pass;

Talysh Mts., Lerik Distr., Gosmalyan

Verhulst, 2000: Colias aurorina aurorina Her. - Schäffer, 1850, Talysh, Alty-Agatch

Sorimachi, 2000: Colias aurorina, Azerbaidzhan

Tschikolovets, Nekrutenko, 2012: Colias aurorina aurorina Herrich- Schäffer, [1850], Talysh, Zuvand; Nakhichevan,

Zangezursky Mts., Bitshenek; Talysh, Zuvand: Gosmolyan and Kal"vaz

Korb, Bolshakov, 2016: Colias aurorina Herrich-Schäffer, [1851], Azerbaijan

71. Colias chlorocoma chlorocoma Christoph, 1888 
Effendi, 1970: Colias chlorocoma Chr., Nakhichevan, Kyarmachatakh (Kərmaçatax)

Didmanidze, 1979: Colias chlorocoma Chr., Nakhichevan, Djulfa surr.

Nekrutenko, 1990: Colias chlorocomma Chr., Nakhichevan

Tuzov, 1993: Colias chlorocoma chlorocoma Christoph, 1888, Armenian plateau

Hasselbarth, van Oorschor \& Wagener, 1995: Colias chlorocoma chlorocoma Christoph, 1888 (= tkatschukovi Bang-Haas, 1936), Nachitschewan

Tuzov, 1997: Colias chlorocoma Christoph, 1888, Nakhichevan Region, Daralagezsky Mts., Buzgov

Verhulst, 2000: Colias chlorocoma tkatschukovi O.Bang-Haas, 1916, Azerbaydzhan, Daralagez Mts., Sultanbek

Sorimachi, 2000: Colias chlorocomma, Azerbaidzhan

Eckweiler, 2003: Colias chlorocoma tkatschukovi, Aserbeidschan SSR, Nachitjevan

Didmanidze, 2004: Colias chlorocoma Christoph, 1888, Nakhichevan, Julfa

S.K.Korb: Colias chlorocoma chlorocoma Christoph, 1888, Nakhichevan Region

Tshikolovets, 2011: Colias chlorocoma Christoph, 1888, Azerbaijan, Nakhichevan

72. Colias chrysotheme chrysotheme (Esper, [1781])

Effendi, 1970: Colias chrysotheme Esp., Shemakha, Pirkuli

Material: 10", Nakhichevan, Agdere, 22.07.2017; 10", Lenkoran, Azfilial,1-4.10.2012

\section{Colias thisoa thisoa Ménétriés, 1832}

Effendi, 1970: Colias thisoa Men., slopes of Baba-Dag, Bazarduzy, Kapaz Mts.

Nekrutenko, 1990: Colias thisoa Mén. (= strandiana Sheljuzhko, 1935), Dzhavakhet-Armenian plateau; Talysh

Tuzov, 1993: Colias thisoa strandiana Sheljuzhko, 1935, Armenian plateau; Talysh

Lukhtanov \& Lukhtanov, 1994: Colias thisoa Ménétriés, 1832, Talysh

Tuzov, 1997: Colias thisoa strandiana Sheljuzhko, 1935, Armenian Highland; Talysh

Sorimachi, 2000: Colias thisoa shakuhensis Sheljuzhko, 1935, Talysh

Verhulst, 2004: Colias thisoa shakuhensis Sheljuzhko, 1935, Talysh

Korb, Bolshakov, 2016: Colias thisoa Ménétriès, 1832, Azerbaijan

\section{Gonepteryx rhamni rhamni(Linnaeus, 1758)}

Ménétriés, 1832: Colias rhamni, Linn., montagnes de Talyche; Zuvant

Lederer, 1870: Rhodocera Rhamni L., Talyche, Zouvent

Christoph, 1886: Rhodocera rhamni L., Lenkoran, Suant

Nekrutenko, 1966: Gonepteryx rhamni transiens Vty., Adzhi-Kent

Nekrutenko, 1968: Gonepteryx rhamni transiens Vty., Adzhi-Kent

Nekrutenko, 1968: Gonepteryx rhamni miljanowskii and transiens transitional form, Zakataly

Effendi, 1970: Gonepteryx rhamni L., everywhere in the foothills and mountains, to the upper boundary of the forest; absent in the lowlands

Didmanidze, 1979: Gonepteryx rhamni L. sbsp. transiens Vtg., road to Baku; Mil steppe, lake Aggel; Turianchay Reserve

Tuzov, 1993: Gonepteryx rhamni meridionalis Rober, 1907, Armenian plateau

Tuzov, 1997: Gonepteryx rhamni meridionalis Röber, 1907, Armenian Highland

Tschikolovets, Nekrutenko, 2012: Gonepteryx rhamni rhamni (Linnaeus, 1758), Gosmolyan; Nakhichevan, Buzgov; Nehram (Nəhrəm), Ms Darydagh (Darıdağ), pr. Dzhulfa

Korb, Bolshakov, 2016: Gonepteryx rhamni(Linnaeus, 1758), Azerbaijan

Material: 19, Nakhichevan, Ordubad, Agdere, 28-30.07.2016; 19, same place, 28.07.2016; 10", same place, 24.07.2017; 19, same place, 22.07.2017; $10^{\prime \prime}$, same place, 9-12.06.2017; 10", 1\%, Nakhichevan, Shakhbuz distr., between the village of Mahmudoba and Nursu, 30.05.2018

\section{Gonepteryx farinosa farinosa (Zeller, 1847)}

Romanoff, 1884: Gonepteryx Rhamnivar. Farinosa Z., Ordoubad; Kedabeg

Effendi, 1970: Gonepteryx farinoza L. - Ordubad, Pazamra, Kedabek

Didmanidze, 1979: Gonepteryx farinosa Celber, Turianchay Reservation; Pirkuli Reservation, Dubrar (Dübrar); Nakhichevan, Naservaz , Araxi river, Arpachay river, Paragachai river

De Freina, 1983: Gonepteryx farinose, Jelisavetpol; Ordoubad; Kedabeg

Tuzov, 1993: Gonepteryx farinose turcirana de Freina, 1982, Armenian plateau

Tuzov, 1997: Gonepteryx farinose turcirana de Freina, 1982, Armenian Highland; Nakhichevan Region, Daralagezsky Mts., Buzgov

Didmanidze, 2004: Gonepteryx farinose Zeller, 1847, Turianchay Reservation; Pirkuli Reservation, Dubrar; Nakhichevan, Naservaz, Araxi river, Arpachay river, Paragachai river

Tschikolovets, Nekrutenko, 2012: Gonepteryx farinosa farinosa (Zeller, 1847), Adzhi-kent; Terter

Korb, Bolshakov, 2016: Gonepteryx farinosa (Zeller, 1847), Azerbaijan 
76. Cigaritis epargyros epargyros (Eversmann, 1854)

Family Lycaenidae [Leach], [1815]

Rühl, [1893]: Cigaritis Acamas Klg. (= Epargyros Eversm.), Lenkoran

Tuzov, 1993: Apharitis acamas marginalis Riley, 1921, Talysh

Zhdanko, 2000: Apharitis epargyros ssp. marginalis (Riley, 1921), Azerbaijan

Bozano \& Weidenhoffer, 2007: Cigaritis (Apharitis) epargyros ssp. marginalis (Riley, 1921), Azerbaijan

Tschikolovets, Nekrutenko, 2012: Cigaritis epargyros epargyros (Eversmann, 1854), Nakhichevan, Ordubad

Korb, Bolshakov, 2016: Cigaritis epargyros (Eversmann, 1854), Azerbaijan

77. Favonius quercus quercus (Linnaeus, 1758)

Christoph, 1877: Thecla quercus L., Lenkoran

Romanoff, 1884: Thecla Quercus L., Adjikent; Kedabeg; Istidara; Lenkoran; Goktcha

Christoph, 1886: Thecla quercus L., Lenkoran

Rühl, [1893]: Thecla quercus L., Lenkoran

Effendi, 1970: Zephyrus quercus L., throughout the mixed forests of the foothills

Tuzov, 1993: Neozephyrus quercus longicauda Riley, 1921, Armenian plateau

Dantchenko, 2000: Favonius quercus ssp. longicaudatus (Riley, 1921), Talysh Mts.

Tschikolovets, Nekrutenko, 2012: Favonius quarcus quercus (Linnaeus, 1758), Nakhichevan, Bitshenek pass.; Kussary

Korb, Bolshakov, 2016: Favonius quercus (Linnaeus, 1758), Azerbaijan

78. Thecla betulae betulae (Linnaeus, 1758)

Lederer, 1870: Thecla betulae L., Elisabethpol

Romanoff, 1884: Thecla Betulae L., Elisabethpol

Effendi, 1970: Zephyrus betulae L., Zakataly, Djari

Korb, Bolshakov, 2016: Thecla betulae (Linnaeus, 1758), Azerbaijan

Material: 1\%, Ismailly Kaladjik, Velasin, 21-23.07.2014

79. Fixsenia pruni pruni (Linnaeus, 1758)

Effendi, 1970: Thecla pruni L., everywhere in the forests of the foothills

80. Fixsenia $w$-album $w$-album (Knoch, 1782)

Romanoff, 1884: Thecla W. Album Knoch., Kedabeg; Adjikent

Effendi, 1970: Thecla w-album Knoch. var. armena Jach., everywhere in the forests of the foothills, rarely in the

mountains

Tuzov, 1993: Nordmannia w-album w-album Knoch, 1782, Armenian plateau; Talysh

Korb, Bolshakov, 2016: Fixsenia w-album (Knoch, 1782), Azerbaijan

Material: 20º', Lerik, Gosmalyan, 21-22.06.2017

\section{Fixsenia ilicis ilicis (Esper, [1779])}

Lederer, 1864: Thecla ilicis Esp., Helenendorf bei Elisabethpol

Peile, 1922: Strymon ilicis caidatula Zell., Lenkoran

Effendi, 1970: Thecla ilicis Esp., Shemakha, Kirovka; Vartashen (now Oguz); Kutkashen (now Gabala); Nukha (now Sheki (Şəki); Zakataly; Kedabek; Lachin; Talysh; Nakhichevan

Didmanidze, 1979: Nordmannia ilicis Esp. sbsp. caudatula Z., Turianchai Reservation; Ordubad

Tuzov, 1993: Nordmannia ilicis ilicis Esper, 1777, Armenian plateau; Talysh

Tschikolovets, Nekrutenko, 2012: Satyrium ilicis ilicis (Esper, [1779]), Elisavetpol

Korb, Bolshakov, 2016: Fixsenia ilicis (Esper, [1779]), Azerbaijan

82. Fixsenia acaciae abdominalis (Gerhard, [1850])

Gerhard, [1850]: Thecla Abdominalis Lederer, Elisabethpol

Lederer, 1864: Thecla abdominalis Gerh., Helenendorf bei Elisabethpol

Romanoff, 1884: Thecla Acaciae F., Adjikent

Effendi, 1970: Thecla acaciae F., everywhere in the foothills, rarely in the mountainsTuzov, 1993: Satyrium abdominalis abdominalis Gerhard, 1850, Armenian plateau; Talysh

Tshikolovets, 2011: Satyrium abdominalis abdominalis (Gerhard, [1850], Nakhichevan, Bichenek

Tschikolovets, Nekrutenko, 2012: Satyrium gerhardi gerhardi (Staudinger, 1895), Nakhichevan, Ordubad distr., Ay-Dara

(Ağdərə) vill.; Djulfa

Tschikolovets, Nekrutenko, 2012: Satyrium abdominalis abdominalis (Gerhard, [1850]), Nakhichevan, Daralgez Mts., Kyukyu; Ordubad distr., Ay-Dara vill.; Talysh-Zuvand, Kosmolian; Nakhichevan, Djulfa; Baku distr.; Kuba

Korb, Bolshakov, 2016: Fixsenia abdominalis (Gerhard, 1882), Azerbaijan 
Material: $1 \sigma^{\prime \prime}$, Shemakha, Demirchi, 24.06.2015; 10, Kedabek, Novoivanovka -Novosaratovka vill., 19-21.06.2014

83. Fixsenia spini spini (Fabricius, 1787)

Ménétriés, 1832: Polyommatus Lyceus, Fab., Lenkoran

Lederer, 1864: Thecla spiniS.V., Helenendorf bei Elisabethpol

Romanoff, 1884: Thecla Spini Schiff. var. Melantho Klug., Ordoubad

Christoph, 1886: Thecla spini Schiff.,var. Lynceus Esp., Lenkoran

Effendi, 1970: Thecla spini Schiff. var. melantha Klug., everywhere in the forests of the foothills

Tuzov, 1993: Nordmannia spini melantho Klug., 1834, Armenian plateau; Talysh

Zhdanko, 2000: Nordmannia spinissp. melantho (Klug., 1834), Armenian Hughland; Talysh

Tuzov, 2000a: Nordmannia spini melantho Klug., Nakhichevan, Buzgov near Ordubad; Pass Bichenek

Tschikolovets, Nekrutenko, 2012: Satyrium spini spini (Fabricius, 1787), Nakhichevan, Zangezur Mts., Bitshenek;

Ms.Talysh, Kosmoljan; val. fl. Dizavar, pr. stat. Kiljazy (Giləzi); Alty-Agatsh; Nakhichevan, Dary-Dagh, Dzhulfa vic.

Korb, Bolshakov, 2016: Fixsenia spini (Fabricius, 1787), Azerbaijan

Material: 20'0', Nakhichevan, Shakhbuz, Kyukyu, 27.07.2016

84. Fixsenia ledereri ledereri (Boisduval, 1848)

Lederer, 1864: Thecla ledereri H. - Sch., Helenendorf bei Elisabethpol

Romanoff, 1884: Thecla Ledereri V., Ordoubad; Helenendorf

Effendi, 1970: Thecla ledereri B., Ordubad, Djulfa, Norashen (now Sharur); Gosmolyan, Kyalvyaz, Kyalyakhan

Didmanidze, 1979: Turianchay Reservetion; Nakhichevan, Djulfa, geyser; neighborhood Bilyav; Araks River Gorge;

Ordubad

Nekrutenko, 1978: Pseudothecla ledereri ledereri Boisduval, Kirovobad (now Gyandja); Akstafa (Ağstafa), Poylu

Tuzov, 1993: Armenia ledereri ledereri Boisduval, 1848, Armenian plateau; Talysh

Tuzov, 2000a: Armenia ledereri ledereri Boisduval, Nakhichevan, Buzgov near Ordubad

Weidenhoffer, 2004: Satyrum (Armenia) ledereri ledereri (Boisduval, 1848), Azerbaidjan

Didmanidze, 2004: Pseudothecla ledereri Boisduval, 1848, Turianchay Reservation; Nakhichevan: Bilav, Ordubad, Araxi gorge, Julfa

Korb, Bolshakov, 2016: Fixsenia ledereri (Boisduval, 1848), Azerbaijan

85. Fixsenia hyrcanicum cyri (Nekrutenko, 1978)

Riley, 1939: Strymon ledereri hyrcanica subsp. nov., Ordubad

Nekrutenko, 1978: Pseudothecla ledereri cyri Nekrutenko, ssp. nov., Khanlar (now Goygel) distr., Enikend (Yenikənd) vill.

Tuzov, 1993: Armenia hyrcanica cyri Nekrutenko, 1978, Armenian plateau

Hesselbarth, van Oorschot \& Wagener, 1995: Satyrium hyrcanicum cyri (Nekrutenko, 1978), Westliches Aserbeidschan,

Nachitschewan

Zhdanko, 2000: Armenia hyrcanica ssp. cyri (Nekrutenko, 1978), Armenian Highland

Tuzov, 2000a: Armenia hyrcanica ssp. cyri Nekrutenko, Nakhichevan, Buzgov near Ordubad; pass Bichenek

Weidenhoffer, 2004: Satyrium (Armenia) hyrcanica cyri Nekrutenko, 1978, Azerbaijan

Tschikolovets, Nekrutenko, 2012: Satyrium hyrcanicum cyri (Nekrutenko, 1978), Kiljazy

Korb, Bolshakov, 2016: Fixsenia hyrcanica cyri (Nekrutenko, 1978), Enikend village, Khanlar distr.

Material: $90^{\prime \prime} 0^{\prime \prime}$ 4우, Nakhichevan, Shakhbuz distr., between the village of Mahmudoba and Nursu, 30.05.2018

86. Callophrys chalybeitincta chalybeitincta Sovinsky, 1905

Lederer, 1864: Thecla rubi L., Helenedorf bei Elisabethpol

Sovinsky, 1905: Callophrys rubi L. var. chalybeitincta nova, Elisabethpol

Jachontov, 1911: Callophrys rubi L. var. (subsp.) chalybeitincta Sovinsky, Aresh (now Khaldan)

Gaede, 1930: Callophrys rubi chalybeitincta Sov. (=caerulescens O.B.-H.), Jelisabethpol

Effendi, 1970: Callophrys chalybaetincta Sov., Kuba; Kusary; Konahkend; Shemakha, Kirovka; Avahil; Lagich; Demirchi;

Kutkashen (now Gabala), Laza; Vartashen (Oguz); Kakhi; Lachin; Mardakert (now Agdere (Ağdərə); Margushevan

(Marguşevan); Talysh, Yardimly; Talysh, Lerik

Tschikolovets, Nekrutenko, 2012: Callophrys rubi chalybeitincta Sovinsky, 1905, Terter (gub.Elisavethpol); Akstafa, Poylu; Jenikend; Zakatala

Korb, Bolshakov, 2016: Callophrys chalybeitincta chalybeitincta Sovinsky, 1905: Transcaucasus

Material: 20"0', Nakhichevan, Shakhbuz, Nursu, 7.05.2018; 10', Shemakha, Demirchi, 24.06.2015

87. Callophrys paulae kolak Higgins, 1965

Tuzov, 1993: Callophrys suaveola kolak Higgins, Talysh

Zhdanko, 2000: Callophrys paulae Pfeiffer, 1932, Talysh Mts.

Zhdanko, 2000: C allophrys paulae ssp. kolak Higgins, 1965, Talysh Mts. (Zuvand Plateau)

Tuzov, 2000b: Callophrys paulae kolak Higgins Talysh Mts., Zuvand Plateau 
Tshikolovets, 2011: Callophrys paulae kolak Higgins, 1965, Nakhichevan

Tschikolovets, Nekrutenko, 2012: Callophrys paulae kolak Higgins, 1965, Nakhichevan, Nehram; Talysh, Galabin (Gələbin) Korb, Bolshakov, 2016: Callophrys paulae Pfeiffer, 1932, Azerbaijan

Material: $60^{\top} \sigma^{\top}$, Nakhichevan, Shakhbuz, Nursu, 7.05.2018; 19, Massali, near Kubin and Mijankyu vill., 5.05.2014

88. Callophrys suaveola danchenkoi Zhdanko, [1999]

Zhdanko, [1999]: Callophrys danchenkoi Zhdanko, sp.n., Nakhichevan, Daralagez Mts., Buzgov

Zhdanko, 2000: Callophrys danchenkoi Zhdanko, 1998, Armenian highland

Tuzov, 2000b: Callophrys danchenkoi Zhdanko, Nakhichevan, Buzgov near Ordubad

Tshikolovets, 2011: Callophrys suaveola danchenkoi Zhdanko, [1999], Nakhichevan, Buzgov

Tschikolovets, Nekrutenko, 2012: Callophrys suaveola danchenkoi Zhdanko, [1999], Nakhichevan, Darashanm (Djulfa)

89. Tomares callimachus callimachus (Eversmann, 1848)

Eversmann, 1848: Lycaena Callimachus, Helenendorf (now Goygol)

Lederer, 1864: Thestor Callimachus Ev., Helenendorf bei Elisabethpol

Lederer, 1870: Thestor Callimachus Ev., Helenendorf

Romanoff, 1884: Thestor Cllimachus Ev., Ordoubad, Alindjachai (əlincaçay)

Rühl, [1893]: Thestor Callimachus Eversm. (= epiphania Boisd.), Ordubat

Effendi, 1970: Thestoe callimachus Ev., Nakhichevan, Paiz, Kermachatakh, Ordubad, Paraga, Pazamara

Tuzov, 1993: Tomares callimachus callimachus Eversmann, 1848, Armenian plateau

Tuzov, 2000b: Tomares callimachus callimachus Eversmann, Nakhichevan, Buzgov near Ordubad

Tshikolovets, 2003: Tomares Callimachus, Alty-Agatsh

Bozano \& Weidenhoffer, 2007: Tomares callimachus (Eversmann, 1848) (= epiphania Boisduval, 1848), Azerbaijan

Tschikolovets, Nekrutenko, 2012: Tomares callimachus callimachus (Eversmann, 1848), Talysh, Zunavd, Everi; Akstafa,

Poylu

Korb, Bolshakov, 2016: Tomares callimachus (Eversmann, 1848), Azerbaijan

90. Tomares desinens desinens Nekrutenko \& Effendi, 1980

Nekrutenko \& Effendi, 1980: Tomares desinens sp.n., Talysh Mts., Zuvand, Hili-dara

Tuzov, 1993: Tomares desinens Nekrutenko et Effendi, 1980, Talysh

Zhdanko, 2000: Tomares desinens Nekrutenko et Effendi, 1980, Zuvand: Gili-dara

Tuzov, 2000b: Tomares desinens Nekrutenko et Effendi, Talysh Mts., Lerik; Zuvand Plateau, Gili-Dara

S.K.Korb, 2005: Tomares desines desinens Nekrutenko \& Effendi, 1980, Talysh

Bozano \& Weidenhoffer, 2007: Tomares desines desinens Nekrutenko \& Effendi, 1980, Talysh

Tshikolovets, 2011: Tomares desines desinens, Talysh Mountains, Zuvand, Hili-Dara

Korb, Bolshakov, 2016: Tomares desinens Nekrutenko et Effendi, 1980, Azerbaijan

\section{Tomares romanovi romanovi(Christoph, 1882)}

Christoph, 1882: Thestor Romanovi n. sp., Ordubat

Romanoff, 1884: Thestor Romanovi Chr., Ordoubad

Rühl, [1893]: Thestor Romanovi Christoph, Ordubat

Staudinger, 1901: Thestor Romanovi Chr., Ordubad

Effendi, 1970: Thestor romanovi Chr., Ordubad, Nyusnyus, Dirnis (Dırnıs), Pazmara

Didmanidze, 1979: Thestor romanovi Christ., Nakhichevan, Ordubad

Eckweiler\&Hofmann, 1980: Tomares romanovissp. romanovi Christoph, 1882, Ordubad

D"Abrera, 1993: Tomares romanovi Christoph, 1881, Ordebad

Tuzov, 1993: Tomares romanovi romanovi Christoph, 1881, Armenian plateau

Zhdanko, 2000: Tomares romanovi romanovi (Christoph, 1882), Armenian Highland

Zhdanko, 2000: Tomares romanovi ssp. cyprius Stichel, 1911, Talysh

Tuzov, 2000b: Tomares romanovi romanovi Christoph, Nakhichevan, Buzgov

van Oorschot \& Wagener, 2000: Tomares romanovi romanovi, Ordubad

Bozano \& Weidenhoffer, 2007: Tomares romanovi romanovi (Christoph, 1882) (= cyprius Stichel, 1911; = maculifera

Staudinger, 1892), Azerbaijan

Tshikolovets, 2011: Tomares romanovi romanovi (Christoph, 1882), Nakhichevan

Tschikolovets, Nekrutenko, 2012: Tomares romanovi romanovi (Christoph, 1882), Nakhichevan, Bilav

Korb, Bolshakov, 2016: Tomares romanovi (Christoph, 1882), Azerbaijan

92. Tomares romanovi cachetinus Nekrutenko, 1978

Van Oorscht \& Wagner, 2000: Tomares romanovi cacheticus, Nekrutenko, 1978, Alty-Agatsh

Tshikolovets, 2011: Tomares romanovi cacheticus, Nekrutenko, 1978, Alty-Agatsh 
93. Tomares nogelii nogelii (Herrich- Schäffer, [1851])

Tshikolovets, 2011: Tomares nogelii nogelii (Herrich- Schäffer, [1851]), mountains south Gandja

Korb, Bolshakov, 2016: Tomares nogelii (Herrich-Schäffer, 1851), Azerbaijan

94. Lycaena phlaeas phlaeas (Linnaeus, 1761)

Ménétriés, 1832: Polyommatus Phlaeas, Linn., Lenkoran

Christoph, 1874: Polyommatus Phlaeas, L., Lenkoran

Romanoff, 1884: Polyommatus Phlaeas, Linn. var. Eleus, Lenkoran; Ordoubad

Christoph, 1886: Polyommatus Phlaeas, L., Lenkoran

Didmanidze, 1979: Lycaena phlaeas L., Steppe Mill, Sari-Su Lake; Nakichevan, Ordubad: border guard, the gorge of the Nakhichevanchay river, Arinj (Arınc), the gorge of the Paragachay River, the Kapujuh ridge, the gorge of the Araks River, Gyvrag, Nus-Nus

Tuzov, 1993: Lycaena phlaeas phlaeas Linnaeus, 1758, Armenian plateau; Talysh

Didmanidze, 2004: Lycaena phlaeas Linnaeus, 1761, Steppe Mili near Lake Sari-Su; Nakhichevan: Ordubad, Mount Koputjukh, Araxi gorge, Kivrak, Nius-Nius

Tschikolovets, Nekrutenko, 2012: Chrysaphanus phlaeas L., Akstafa, Poylu; Ms.Talysh, Lerik, Gosmolyan, Hili-Dara; AdzhiKent; Elisavetpol

Korb, Bolshakov, 2016: Lycaena phlaeas (Linnaeus, 1760), Azerbaijan

Material: $10^{\prime \prime}$, Nakhichevan, Ordubad, Agdere 27.07.2018; 10", 19, same place, 21.07.2017; $10^{\circ}$, same place, 22.07.2017; 10", Zakatala State Reserve, Akhkemal, 11.06.2015; 10;, Lerik, Gosmalyan, 21-22.06.2017; 10;, Kedabek,Novosaratovka, 6-

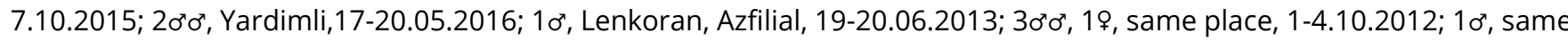
place, 26-28.05.2014; $1 \sigma^{\prime \prime}$, Zakatala-Balaken, 1-4.04.2014; 10', Astara,Toradi, 13-16.06.2016; 10", 19, Zakatala, Djidjikhana, 10-12.07.2015; 10; 19, Zakatala, from kombinat to State Reserve, 24.06.2014; 200', Belokani, Beshbulag, Djidjikhana, 25.06.2013; 10", 2\%क, Lenkoran, Azfilial, 16-19.09.2013; 10", 1\%, Massali, near Kubin and Mijankyu vill., 5.05.2014

\section{Lycaena tityrus tityrus (Poda, 1761)}

Effendi, 1970: Chrysophanus dorilis Hufn. ssp. orientalis Stgr., everywhere

Didmanidze, 1979: Lycaena tityrus Pod. (= Ch.dorilis Hufn.) sbsp.orientalis Stgr., Nakhichevan, Ordubad, Paragachay river gorge, Kapudjik Mt.

Tuzov, 1993: Heodes tityrus orientalis, Staudinger, 1881, Armenian plateau

Tuzov, 1993: Heodes tityrus opisthochros Verity, 1939, Talysh

Didmanidze, 2004: Lycaena tityrus Poda, 1761, Nakhichevan: Paragachai, Mount Kopujukh

Tschikolovets, Nekrutenko, 2012: Lycaena tityrus tityrus (Poda, 1761), Nakhichevan, Ordubad: Pazmara vill.; Zangezur Mts., Bitshenek; Adzhi-Kent

Korb, Bolshakov, 2016: Lycaena tityrus (Poda, 1761), Azerbaijan

Material: 30"0", Zakatala, Djidjikhana, 10-12.07.2015; 10, 1\%, Belokani, Beshbulag, Djidjikhana, 25.06.2013

96. Lycaena japhetica japhetica Nekrutenko \&Effendi, 1983

Nekrutenko \& Effendi, 1983: Lycaena japhetica Nekrutenko et Effendi, sp.n., Apsheron distr., Dizavartshaj (Dizəvarçay) river valley (between Kilyazy station and Tazakend village)

D"Abrera, 1993: Lycaena japhetica japhetica Nekrutenko \& Effendi, 1983, inter Kilyazi and Tazakend

Tuzov, 1993: Athamantia japhetica Nekrutenko et Effendi, 1983, Small Caucasus, Azerbaijan

Zhdanko, 1993: Athamanthia japhetica japhetica Nekrutenko, 1983, E. Azerbaijan

Lukhtanov, 2000: Athamantia japhetica (Nekrutenko et Effendi, 1983), Disavartschai (zwischen Kiljasi und Tasakend), Apscheron Bezirk)

Zhdanko, 2000: Athamantia japhetica (Nekrutenko et Effendi, 1983), the type locality in Azerbaijan

Tuzov, 2000b: Athamantia japhetica Nekrutenko et Effendi, Tazakend, Dizavarchai River; Tazakend, Kilyazi

Bozano\&Weidenhoffer, 2001: Athamantia japhetica (Nekrutenko et Effendi, 1983), E. Azerbaijan, Alty-Agatch; Chyzy (Xızı)

Tshikolovets, 2003: Lycaena japhetica, Tazakend

Tshikolovets, 2011: Lycaena japhetica japhetica Nekrutenko \& Effendi, 1983, arid ranges around western part of Caspian Sea in extreme N. - E. Azerbaijan, Dizavarchay River

Korb, Bolshakov, 2016: Lycaena japhetica japhetica (Nekrutenko et Effendi, 1983), NE Azerbaijan

\section{Lycaena thersamon thersamon (Esper, [1784])}

Romanoff, 1884: Polyommatus Thersamon Esp., Ordoubad

Effendi, 1970: Chrysaphanus thersamon Esp., throughout the lowlands and in the foothills

Didmanidze, 1979: Thersamonia thersamon Esp. Nalhichevan, Paragachay river gorge, Nakhichevanchay river gorge,

Shakhbuz Ridge

Tuzov, 1993: Thersamonia thersamon kurdistanica Riley, 1921, Armenian plateau; Talysh

Tuzov, 2000b: Thersamonia kurdistanica Riley, Nakhichevan, Kyukyu; Pass Buchenek; Ordubad 
Didmanidze, 2004: Thersamonia thersamon Esper, 1784, Nakhichevan, Paragachai gorge near Kivrak, Mount Shakhbus Tschikolovets, Nekrutenko, 2012: Lycaena thersamon thersamon (Esper, [1784]), Nakhichevan, Zangezursky Mts., Bitshenek; Traj Bitshenek; Shachbuz; Mingetshaur; Ms.Talysh, Lerik; Elisavetpol; Terter Korb, Bolshakov, 2016: Lycaena thersamon (Esper, [1784]), Azerbaijan Material: 19, Nakhichevan, Ordubad, Agdere, 22.07.2017; 10, Absheron, Zabrat, 22.06.2013

98. Lycaena asabinus asabinus (Herrich- Schäffer, [1851])

Romanoff, 1884: Polyommatus Asabinus HS. ver. Satraps Stgr., Ordoubad

Effendi, 1970: Chrysophanus asabinus HS. ssp. satraps Stgr., Talish, Yardimli, Istisu, Lerik, Lyulyakeran; Nakhichevan, Ordubad, Dirnis (Dırnıs)

Tuzov, 1993: Thersamonia asabinus satraps Staudinger, 1879, Armenian plateau; Talysh

Tshikolovets, 2011: Lycaena asabinus (Herrich - Schäffer, [1851]), Nakhichevan, Paragachay

Tschikolovets, Nekrutenko, 2012: Lycaena asabinus asabinus (Herrich - Schäffer, [1851]), Talysh-Zuvand, Kosmoljan

Korb, Bolshakov, 2016: Lycaena asabina Herrich-Schäffer, 1851, Azerbaijan

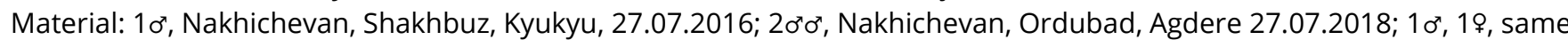
place, 11.06.2017

99. Lycaena ochimus ochimus (Herrich- Schäffer, [1851])

Effendi, 1970: Chrysaphanus ochimus HS, Talush, Kyalyakhan, Kizyurdi Mt.; Nakhichevan, Ordubad, Pazamara

Nekrutenko, 1977: Heodes (Thersamonia) ochimus ochimus, Azerbaijan

Tuzov, 1993: Thersamonia kefersteini kefersteini Gerhard, 1850, Armenian plateau

Tuzov, 1993: Thersamonia kefersteini sachandensis Schurian et Hoffman, 1982, Talysh

Samodurov \& Zhdanko, 2000: Thersamonia ochomus ochimus (Herrich- Schäffer, [1852]), Armenian Highland

Samodurov \& Zhdanko, 2000: Thersamonia ochomus ssp. sachandensis Schurian et Hoffmann, 1982, Talysh Mts.

Tschikolovets, Nekrutenko, 2012: Lycaena ochimus ochimus (Herrich - Schäffer, [1851]), Talysh-Zuvand, Kosmoljan \& Galabin

Korb, Bolshakov, 2016: Lycaena ochimus (Herrich-Schäffer, 1851), Azerbaijan

100. Lycaena dispar rutila (Werneburg, 1864)

Effendi, 1970: Chrysaphanus dispar Hw. ssp. rutilus Wernb., throughout the lowlands and in the foothills, in open areas Tuzov, 1993: Thersamonolycaena dispar rutilus Werneburg, 1864, Armenian plateau; Talysh

Tschikolovets, Nekrutenko, 2012: Lycaena dispar rutile (Werneburg, 1864), Nakhichevan, Nus-Nus, prope Ordubad

Korb, Bolshakov, 2016: Lycaena dispar ([Haworth], 1802), Azerbaijan

Material: 19, Belokani, Djidjikhana, 25.06.2013

101. Lycaena alciphron melibaeus (Staudinger, 1878)

Romanoff, 1884: Polyommatus A/ciphron Rott. var. Melibaeus Stgr, Ordoubad, Adjikent, Istisou

Effendi, 1970: Chrysaphanus alciphron Rott., Shemakha, Pirkuli; Kutkashen (now Gabala), Laza; Lachin, Yanshakh; Nukha (now Sheki), Topchi; Nakhichevan, Shakhbuz

Didmanidze, 1979: Palaeochrysophanus alciphron Rott. sbsp. gordius Vrty., Nakhichevan, Kivrak, border guard

D"Abrera, 1993: Lycaena alciphron fruginus Fruhstorfer, Ordubad

Tuzov, 1993: Thersamonolycaena alciphron fruginus Fruhstorfer, 1917, Talysh

Didmanidze, 2004: Heodes alciphron Rotterburg, 1775, Nakhichevan, Ordubad, Kivrak

Korb, 2005: Thersamonolycaena alciphron fruginus Fruhstorfer, 1917, Talysh

Tschikolovets, Nekrutenko, 2012: Lycaena alciphron melibaeus (Staudinger, 1878), Nakhichevan, Ordubad disrt., Pazmara vill., Buzgov

Korb, Bolshakov, 2016: Lycaena alciphron (Rottemburg, 1775), Azerbaijan

Material: 10", Nakhichevan, Ordubad, Agdere, 28-30.07.2016; 19, same place, 23-24.07.2018; 10", same place, 11.06.2017; 19, Belokani, Beshbulag, Djidjikhana, 25.06.2013

102. Lycaena virgaureae virgaureae (Linnaeus, 1758)

Ménétriés, 1832: Polyommatus virgaureae, Linn., Talyche, Zouvant

Lederer, 1864: Polyommatus virgaureae, L., Helenendorf bei Elisabethpol

Romanoff, 1884: Polyommatus Virgaureae L., Kedabeg

Jachontov, 1911: Chrysophanus virgaureae L., Zakataly, Akh-Bulag Mts.

Effendi, 1970: Chrysaphanus virgaureae L., everywhere in the foothills and mountains up to $2400 \mathrm{~m}$

Tuzov, 1993: Heodes virgaureae armeniaca A. Bang-Haas, 1906, Armenian plateau

Samodurov, 2000: Heodes virgaureae ssp. armeniaca (O.Bang-Haas, 1906), Armenian Highland

Zhdanko, 2002: Heodes virgaureae armeniaca (O.Bang-Haas, 1906), Armenian Highland

Tschikolovets, Nekrutenko, 2012: Lycaena virgaureae virgaureae (Linnaeus, 1758), Nakhichevan, Daralagez Mts., Kyukyu vill.; Ordubad distr., Pazmara vill.; Traj Bitshenek 
Korb, Bolshakov, 2016: Lycaena virgaureae (Linnaeus, 1758), Azerbaijan

Material: $10^{\prime}$, Nakhichevan, Agdere, 24.07.2017

103. Lycaena thetis thetis Klug, 1834

Effendi, 1970: Chrysaphanus thetis Klug.caudatus Stgr., Norashen (now Sharur), the slopes of Velidag Mt.; Ordubad, Kyalaki

Tuzov, 1993: Thersamonia thetis ignites Herrich-Schaffer, 1851, Armenian plateau

Samodurov \& Zhdanko, 2000: Thersamonia thetis (Klug, 1834) (= ignites Herrich-Schäffer, 1851; = caudatus Staudinger, $1901 ;=$ zahaltensis Graves, 1910), Armenian Highland

Tuzov, 2000b: Thersamonia thetis thetis Klug., Nakhichevan, Nyusnyus

Tshikolovets, 2011: Lycaena thetis Klug., 1834, Nakhichevan

Tschikolovets, Nekrutenko, 2012: Lycaena thetis thetis Klug, 1834, Nakhichevan, Ordubad distr., Pazmara; Paragatshai fl. val.; Ms.Talysh, Kosmoljan; Zuvant, Tatoni vill.

Korb, Bolshakov, 2016: Lycaena thetis Klug, 1834, Azerbaijan

104. Lycaena candens candens (Herrich- Schäffer, [1844])

Lederer, 1864: Polyommatus hippoyhoe L., Helenendorf bei Elisabethpol

Effendi, 1970: Chrysaphanus hippothoe L., slopes of Babadag, Shahdag, Bazarduzu Mts.

Tschikolovets, Nekrutenko, 2012: Lycaena candens candens (Herrich- Schäffer, [1844]), Nakhichevan, Zangezur Mts.,

Bitshenek vill.; Ordubad, Alakhi vill.

Korb, Bolshakov, 2016: Lycaena candens (Herrich-Schäffer, [1844]), Azerbaijan

Material: $10^{\prime \prime}$, Nakhichevan, Ordubad, Agdere, 28.07.2016; 10', same place, 21.07.2017

105. Athamanthia phoenicurus transcaucasicus (Miller, 1923)

Effendi, 1970: Chrysophanus phoenicurus Ld. ssp. transcaucasus Mill., Nakhichevan, Djakhri (Cahri), Paiz, Ordubad, Kyalyaki (Kələki)

Nekrutenko, 1985b: Lycaena melicertes Nekrutenko, sp. n., Nakhichevan, Arax river valley, Negram vill.

Zhdanko, 1993: Athamanthia phoenicurus Lederer, 1871 (= melicertis Nekrutenko, 1985), Nakhichevan

Zhdanko, 2000: Athamanthia phoenicurus transcaucasica (Miller, 1923), Nakhichevan

Tuzov, 2000b: Athamanthia phoenicurus melicertis Nekrutenko et Effendi, Nakhichevan, Negram

Tshikolovets, 2011: Lycaena phoenicura transcaucasica (Miller, 1923) (= melicertis Nekrutenko, 1985), Nakhichevan

Korb, Bolshakov, 2016: Athamanthia phoenicurus transcaucasicus (Miller, 1923): Transcaucasus

106. Lampides boeticus boeticus (Linnaeus, 1767)

Effendi, 1970: Lampides (Polyommatus) boeticus L., Shemakha, Kirovka, Chukhuryurd, Astrakhanka; Kutkashen (now Gabala), Boom; Lerik, Barzavu; Ordubad, Nus-Nus

Tuzov, 1993: Lampides boeticus Linnaeus, 1767, Armenian plateau; Talysh

Koçak, 1996: Lampides boethicus (Linnaeus, 1767), Azerbayejan (Azerbaijan)

Tschikolovets, Nekrutenko, 2012: Lampides boeticus boeticus (Linnaeus, 1767), Nakhichevan, Ordubad

Korb, Bolshakov, 2016: Lampides boeticus (Linnaeus, 1767), Azerbaijan

107. Leptotes pirithous pirithous (Linnaeus, 1767)

Effendi, 1970: Lampides telicanus Lang., Apsheron - Botanical Garden, Mashtagi, Bilga; Agdash,Garagan; Agsu;

Geokchay; Bozdag Hill System

Tuzov, 1993: Leptotes pirithous pirithous Linnaeus, 1767, Talysh

Tuzov, 2000b: Leptotes pirithous ssp.pirithous (Linnaeus, 1767), Lenkoran

Korb, Bolshakov, 2016: Leptotes pirithous (Linnaeus, 1767), Azerbaijan

108. Tarucus balkanicus balkanicus (Freyer, [1844])

Romanoff, 1884: Lycaena Balkanica Frr., Zangesour

Evans, 1955: Tarucus balkanica areshana Bethune-Baker, 1918: Ordubad

Effendi, 1970: Lampides (Tarucus) balcanica Frr., Absheron, Bilgah, Zagulba, Baku (Botanical Garden); Gobustan, Aghsu Pass

Tuzov, 1993: Tarucus balcanicus balcanicus Freyer, 1884, Armenian plateau; Talysh

Koçak, 1996: Tarucus balkanicus ssp. areshanus B.-B., 1918, Nahcivan (Nakhichevan)

Tuzov, 2000a: Taracus balcanicus balcanicus (Freyer, [1844]), Armenian Highland; Talysh Mts.

Tschikolovets, Nekrutenko, 2012: Tarucus balkanicus balkanicus (Freyer, [1844]), Akstafa; Turianchay State Reserve Korb, Bolshakov, 2016: Tarucus balkanicus (Freyer, 1844), Azerbaijan

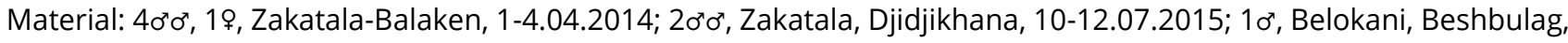

Djidjikhana, 25.06.2013 
109. Lachides galba galba (Lederer, 1855)

Jachontov, 1913: Chilades phiala Gr.-Gr., Kyurdamir, Shemakhan distr.

Effendi, 1970: Lampides (Chilades) phiala Gr. Gr., Absheron, Kobustan, Agsu Pass; Kurdamir (Kürdəmir); Araks Valley

Nekrutenko, 1984: Lachides galba galba(Lederer), comb. n., Salyany; Evlakh (Evlax)

Tuzov, 1993: Lachides galba galba Lederer, 1855, Talysh

Tuzov, 2000b: Lachides galba galba Lederer, Saliyany

Tuzov, 2000a: Lachides galba galba (Lederer, 1855), Talysh Mts.

Tschikolovets, Nekrutenko, 2012: Lachides galba galba (Lederer, 1855), Talysh, Lerik

Korb, Bolshakov, 2016: Lachides galba (Lederer, 1855), Azerbaijan

110.Chilades trochylus trochylus (Freyer, [1845])

Romanoff, 1884: Lycaena trochilus Frr., Ordoubad

Effendi, 1970: Chiledes (Freyeria) trochylus Frr., Talish, Astara, Artupa

Tuzov, 1993: Freyeria trochylus Freyer, 1845, Armenian plateau, Talysh

Tuzov, 2000a: Freyeria trochylus ssp. trochylus (Freyer, [1845]), Armenian Highland, Azerbaijan

Didmanidze, 2004: Chilades trochylus Freyer, 1845, Nakhichevan, Ordubad, Julfa

Tshikolovets, 2011: Chilades trochylus trochylus (Freyer, [1845]), S.Azerbaijan

Tschikolovets, Nekrutenko, 2012: Chilades trochylus trochylus (Freyer, [1845]), Mts.Talysh-Zuvand, Kosmoljan, Divagatsh; Arax fl., Dzhuga; Arax fl., Norashen (now Sharur); Arax fl., Negram; Ordubad

Korb, Bolshakov, 2016: Freyeria trochylus (Freyer, 1845), Azerbaijan

111.Celastrina argiolus argiolus (Linnaeus, 1758)

Ménétriés, 1832: Polyommatus Argiolus, Linn., Lenkoran

Lederer, 1870: Lycaena Argiolus L., Lenkoran

Christoph, 1874: Lycaena Argiolus L., Lenkoran

Christoph, 1886: Lycaena Argiolus L., Lenkoran

Rühl, [1893], Lycaena Argiolus L., Lenkoran

Effendi, 1970: Cyaniris argiolus L., throughout the lowlands and in the foothills

Didmanidze, 1979: Celastrina argiolus L., Turianchay Reserve; Mil steppe, Saatly; Nakhichevan: Nakhichevanchay River gorge, Arinj, Kyukyu neighborhood, Bilav

Tuzov, 1993: Celastrina argiolus argiolus Linnaeus, 1758, Armenian plateau; Talysh

Tuzov, 2000a: Celastrina argiolus argiolus (Linnaeus, 1758), Armenian Highland; Talysh Mts.

Didmanidze, 2004: Celastrina argiolus Linnaeus, 1758, Turianchay Reservation, Saatli steppe Mili; Nakhichevan: Arinji, KiuKiu, Bilav

Tschikolovets, Nekrutenko, 2012: Celastrina argiolus argiolus (Linnaeus, 1758), Mingetshaur; Talysh; Traj.Bitshenek; Elisavetpol

Korb, Bolshakov, 2016: Celastrina argiolus (Linnaeus, 1758), Azerbaijan

Material: 20'0', Nakhichevan, Ordubad, Agdere 23-24.07.2018; 10', same place, 22.07.2017; $10^{\prime \prime}$, Gabala, near Vendam and Bunud, 5-8.07.2016; $10^{\pi}$, Lenkoran, Azfilial, 19-20.06.2013

112. Everes argiades argiades (Pallas, 1771)

Christoph, 1874: Lycaena Argiades Pall., Lenkoran

Christoph, 1886: Lycaena Argiades Pall., Lenkoran

Rühl, [1893]: Lycaena Argiades Pall., Lenkoran

Effendi, 1970: Everes (Lycaena) argiades Pall., throughout the lowlands and in the foothills

Didmanidze, 1979: Everes argiades Ball. sbsp.polysperchon Berg., Turianchai Reservation; Sumgait (Sumgayıt) Tuzov, 1993: Everes argiades (Pallas, 1771), Talysh

Didmanidze, 2004: Everes argiades Pallas, 1771, Turianchay reservation

Tschikolovets, Nekrutenko, 2012: Everes argiades argiades (Pallas, 1771), Elisavetpol

Korb, Bolshakov, 2016: Cupido argiades (Pallas, 1771), Azerbaijan

Material: $1 \sigma^{\prime \prime}$, Lenkoran, Azfilial, 25-28.05.2013

113. Cupido minimus minimus (Füessly, 1775)

Effendi, 1970: Lycaena (Zizera) minima Fuessl., everywhere in the lowlands and foothills

Tschikolovets, Nekrutenko, 2012: Cupido minimus minimus (Füessly, 1775); Alty-Agatsh

Korb, Bolshakov, 2016: Cupido minimus (Fuessly, 1775), Azerbaijan

114. Cupido osiris osiris (Meigen, [1829])

Effendi, 1970: Lycaena sebrus B., everywhere in the lowlands and foothills 
Didmanidze, 1979: Cupido osiris Meig., Turianchai Reservation; Nakhichevan, Bilav

Tuzov, 1993: Cupido osiris majuspunctata Verity, 1934, Talysh

Didmanidze, 2004: Cupido osiris osiris Meigen, 1829, Turianchai reservation; Nakhichevan, Neservaz, Bilav

S.K.Korb, 2005: Cupido osiris majuspunctata Verity, 1934, Talysh plateau

Tschikolovets, Nekrutenko, 2012: Cupido osiris osiris (Meigen, [1829]), Alty-Agatsh; Nakhichevan, Paragatshaj val.;

Elisavetpol

Korb, Bolshakov, 2016: Cupido Osiris (Meigen, 1829), Azerbaijan

Material: 10', Nakhichevan, Ordubad, Agdere, 11.06.2017; 10, 19, Ismailly Kaladjik, Velasin, 21-23.07.2014

\section{Turanana endymion endymion (Freyer, [1850])}

Romanoff, 1884: Lycaena Panagaea HS., Ordoubad

Effendi, 1970: Lycaena panagaea HS, Astara, Artupa; Lenkoran, tea farm "Aurora"

Didmanidze, 1979: Turanana panageae HS., Nakhichevan, Ordubad

Tuzov, 1993: Turanana endymion ahasferi Bytinski-Salz, 1937, Armenian plateau; Talysh

Zhdanko, 2000: Turanana endymion endymion (Freyer, [1850]), Armenian Highland; Talysh

Tuzov, 2000b: Turanana endymion endymion Freyer, Talysh Mts., Zuvand Plateau, Gosmolyan

Didmanidze, 2004: Turanana panagaea Herrich-Shäffer, 1851, Nakhichevan, Ordubad

Tshikolovets, 2011: Turanana endymion (Freyer, [1850]), S. Azerbaijan

Tschikolovets, Nekrutenko, 2012: Turanana endymion endymion (Freyer, [1850]), Talysh, Lerik distr., Kishlak vill.;

Mts.Talysh, Hili-Dara; Lerik vic.; Talysh, Zuvand; Verknyaya Amburdarya (Yuxarı Amburdərə)

Korb, Bolshakov, 2016: Turanana endymion (Freyer, [1850]), Azerbaijan

\section{Iolana iolas lessei Bernardi, 1964}

Tuzov, 1993: Iolana iolas iolas Ochsenheimer, 1816, Armenian plateau; Talysh

Tuzov, 2000 a: Iolana iolas ssp. lesseri (Bernardi, 1964), Armenian Highland; Talysh Mts.

Tuzov, 2000 b: Iolana iolas lesseri Bernardi, Talysh Mts., Veri

Tshikolovets, 2011: Glaucopsyche iolas lesseri Bernardi, 1964, S. Azerbaijan

117.Glaucopsyche alexis alexis (Poda, 1761)

Ménétriés, 1832: Polyommatus Alexis, Wien, Talyche

Romanoff, 1884: Lycaena Cyllarus Rott., Ordoubad; Istissou (İstisu)

Effendi, 1970: Lycaena cyllarus Rott. ssp. aeruginosa Stgr., everywhere in the forest plots of the foothills, at least - in the lowlands

Didmanidze, 1979: Glaucopsyche alexis Poda (= cyllarus Rott.), Nakhichevan, Naservaz; Nakhichevanchay river gorge, Arindj Tuzov, 1993: Glaucopsyche alexis lugens Caradja, 1893, Armenian plateau

Didmanidze, 2004: Glaucopsyche alexis Poda, 1761, Nakhichevan, Naservaz, Aridj

Tschikolovets, Nekrutenko, 2012: Glaucopsyche alexis alexis (Poda, 1761), Daralagez Mts., Buzgov; Akstafa, Pojlu Korb, Bolshakov, 2016: Glaucopsyche alexis (Poda, 1761), Azerbaijan

Material: $1 \sigma^{\prime \prime}$, Nakhichevan, Shakhbuz distr., between the village of Mahmudoba and Nursu, 30.05.2018; $10^{\prime \prime}$, Nakhichevan, Ordubad, Agdere, 9-12.06.2017

\section{Phengaris alcon monticola (Staudinger, 1901)}

Effendi, 1970: Lycaena alcon F. ssp. monticola Stgr., Shemakha, Chukhuryurd, Astrakhanka; Ismailly (İsmayıllı)I Nukha (now Sheki)

Tuzov, 1993: Maculinea rebeli rebeli Hirschke, 1904, Armenian plateau

Tuzov, 2000a: Maculinea rebelissp. monticola (Staudinger, 1901), Armenian Highland)

Tschikolovets, Nekrutenko, 2012: Maculinea alcon monticola (Staudinger, 1901), Elisavetpol; Nakhichevan, Daralagez Mts., Bitshenek

Korb, Bolshakov, 2016: Phengaris alcon ([Denis et Schiffermüller], 1775), Azerbaijan

119. Phengaris teleius teleius (Bergsträsser, 1779)

Ménétriés, 1832: Polyommatus Euphemus, Hubn., Talyche

Lederer, 1870: Lycaena Euphemus Hb., Lenkoran

Christoph, 1886: Lycaena Euphemus Hb., Talysh

Korb, Bolshakov, 2016: Phengaris teleius (Bergsträsser, 1779), Azerbaijan

120.Phengaris arion arion (Linnaeus, 1758)

Ménétriés, 1832: Polyommatus Arion, Linn., Lenkoran

Lederer, 1870: Lycaena Arion L., Lenkoran

Christoph, 1886: Lycaena Arion L., Lenkoran 
Effendi, 1970: Lycaena arion L. ssp. obscura Chr., Shemakha, Avakhil, Meysari, Kyalyakhana (Kələxana); Vartashen (now Oguz)

Korb, Bolshakov, 2016: Phengaris arion (Linnaeus, 1758), Azerbaijan

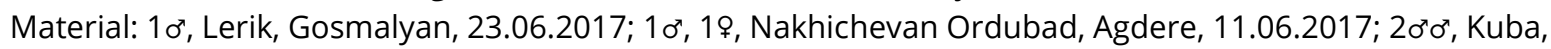

Afurdja, 8.07,2019

\section{Pseudophilotes bavius bavius (Eversmann, 1832)}

Tschikolovets, Nekrutenko, 2012: Pseudophilotes bavius bavius (Eversmann, 1832), Alty-Agatsh

Korb, Bolshakov, 2016: Pseudophilotes bavius (Eversmann, 1832), Azerbaijan

Material: 10, Absheron, Zabrat, 22.06.2013

\section{Pseudophilotes vicrama schiffermuelleri (Hemming, 1929)}

Effendi, 1970: Lycaena (Philotes) vicrama Moore (= baton Brgst ssp. clara Srgr.), Absheron and further south along the coast to Port Ilyich (now Liman)

Nekrutenko, 1977: Pseudophilotes vicrama schiffermuelleri Hemming, (1929), Nakhichevan, Paragatshai river valley

Tuzov, 1993: Pseudophilotes vicrama schiffermuelleri Hemming, 1929, Armenian plateau; Talysh

Didmanidze, 2004: Pseudophilotes vicrama Moore, 1865, Turianchai Reservation; Nakhichevan: Paragachai gorge, Kivrak, Julfa

Tschikolovets, Nekrutenko, 2012: Pseudophilotes vicrama schiffermuelleri (Hemming, 1929), Elisavetpol

Korb, Bolshakov, 2016: Pseudophilotes vicrama (Moore, 1865), Azerbaijan

Material: 10", Absheron, Zabrat, 22.06.2013

123. Plebejus argus aegidion (Meisner, 1818)

Ménétriés, 1832: Polyommatus Argus, Linn., Lenkoran

Ménétriés, 1932: Polyommatus Aegon, Wien., Lenkoran

Christoph, 1886: Lycaena Aegon Sch., Lenkoran

Christoph, 1886: Lycaena Argus Sch., Lenkoran

Effendi, 1970: Lycaena argeis L.ssp. bella HS (= aegon Schiff.), everywhere in the foothills and lowlands, with the exception of saline semi-deserts

Didmanidze, 1979: Lycaena argus sbsp. bella HS., Mil steppe, environs Zhdanovsk; Divichi (Dəvəçi) (now Shabran

(Şabran), Primorye; Nakhichevan: Araks river gorge; Ordubad environs, Araks valley, tugai floodplain

Tuzov, 1993: Plebejus argus bella Herrich-Shaffer, 1843, Armenian plateau

Tuzov, 1993: Plebejus argus orientaloides Verity, Talysh

Koçak, 1996: Plebejus argus ssp. tscherkessica Forst., 1936 (= georgica Forst., 1936), Azerbayejan

Tuzov \& Dantchenko, 2000: Plebejus argus (Linnaeus, 1758), Armenian Highland; Talysh

Tschikolovets, Nekrutenko, 2012: Plebejus argus aegidion (Meisner, 1818), Adzhi-kent (prope Elisavetpol)

Korb, Bolshakov, 2016: Plebejus argus (Linnaeus, 1758), Azerbaijan

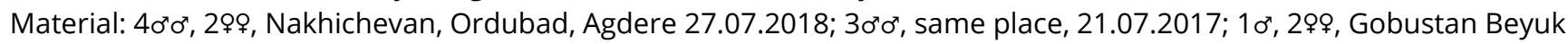

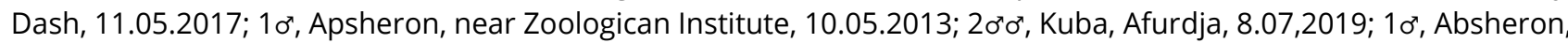

Zabrat, 22.06.2013

\section{Plebejus christophi transcaucasicus (Rebel, 1901)}

Tuzov, 1993: Lycaeides christophi transcaucasicus Rebel, 1901, Talysh

Olivier, van der Poorten \& W. De Prins, 1998: Plebejus christophi transcaucasicus (Rebel, 1901), Jenikend, 50 km N Gjandzha; Kura valley, 50-60 km N. Mingetshaur, Palan-Tiukan

Tshikolovets, 2011: Plebejus christophi transcaucasicus (Rebel, 1901), Nakichevan

Tschikolovets, Nekrutenko, 2012: Plebejus christophi transcaucasicus (Rebel, 1901), Elisavetpol, stat.Evlach

Korb, Bolshakov, 2016: Plebejus christophi(Staudinger, 1874), Azerbaijan

Material: 10", 1\%, Nakhichevan, Ordubad, Agdere, 9.06.2017; 10', Yardimli, 17-20.05.2016; 5m, 2\%o, Shemakha, Demirchi, 24.06.2015

\section{Plebejus idas baldur (Hemming, 1934)}

Tuzov, 1993: Lycaena idas altarmena Forster, 1936, Talysh

Korb, Bolshakov, 2016: Plebejus idas (Linnaeus, 1760), Azerbaijan

Material: 10 m, Bilyasuvar, near road, 18.07.2015; 19, Kedabek, Novosaratovka, 6-7.10.2015

\section{Plebejus artaxerxes allous (Geyer, [1836])}

Tuzov, 1993: Aricia allous sheljuzhkoiObraztsov, 1935, Talysh

Koçak, 1996: Polyommatus (Aricia) artaxerxes ssp., Azerbayejan

Korb, Bolshakov, 2016: Aricia artaxerxes (Fabricius, 1793), Azerbaijan 
Korb, Bolshakov, 2016: Aricia artaxerxes (Fabricius, 1793), Azerbaijan

127.Plebejides sephirus sephirus (Frivaldszky, 1835)

Romanoff, 1884: Lycaena Zephyrus Friv., Helenendorf

Effendi, 1970: Lycaena (Polyommatus) zephyrus Friv., Lachin, Zorkeshish; Ordubad, Unus

Tshikolovets, 2011: Plebejus sephirus sephirus (Frivaldszky, 1835), W. Azerbaijan

Korb, Bolshakov, 2016: Plebejus sephirus (Frivaldszky, 1835), Azerbaijan

128. Plebejides zephyrinus zephyrinus (Christoph, 1884)

Romanoff, 1884: Lycaena Zephyrinus Friv., Ordoubad

Forster, 1938: Lycaena (Plebejus) pylaon ordubadissp. nov., Ordubad

Didmanidze, 1979: Plebejus Zephyrinus Chr., Mil steppe, Aggel lake; Nakhichevan, Paragachai river gorge, Nyusnyus Bálint, Kertézs \& Lukhtanov, 1993: Plebejus sephirus semiturcmenicus Bálint, 1991, Azerbaijan

Tuzov, 1993: Plebejus sephirus semiturcmenicus Bálint, 1991, Armenian plateau; Talysh

Hesselbarth, van Oorschot \& Wagener, 1995: Plebejus (Plebejides) pylaon turkmenicus (Forster, 1936) (= ordubadi Forster, 1938 syn. nov.), Nakhitshevan

Koçak, 1996: Plebejus (Plebejides) zephyrinus ssp. ordubadi Forst., 1938, Nahcivan

Samodurov, Zhdanko \& Tuzov, 2000: Plebejus (Plebejides) zephyrinus ssp. ordubadi (Forster, 1938) (= alberti Nekrutenko, 1975); = semiturcmenicus Bálint, 1991; = abchasicus Nekrutenko, 1975), Armenian Highland; Talysh

Zhdanko, 2002: Plebejides zephyrinus ssp. ordubadi Forster, 1938 (= alberti Nekrutenko, 1975), Armenian Highland; Talysh

Tschikolovets, Nekrutenko, 2012: Plebejus zephyrinus zephyrinus (Christoph, 1884), Ms.Talysh-Zuvand, Gosmoljan; Kolvaz; Nakhichevan, Bitshenek

Korb, Bolshakov, 2016: Plebejus zephyrinus (Christoph, 1884), Azerbaijan

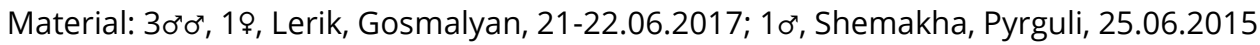

\section{Plebejidea loewii loewii (Zeller, 1847)}

Romanoff, 1884: Lycaena Loewii Z., Ordoubad

Effendi, 1970: Lycaena lowevii L. (= empyrea Frr.), Talysh, Kosmolyan; Nakhichevan, Ordubad, Kyalyaki

Didmanidze, 1979: Plebejus loewii Z., Baku neighborhood; Nakhichevan, Ordubad

Tuzov, 1993: Plebejidea loeweii loewii Zeller, 1847, Armenia plateau

Tuzov, 1993: Plebejidea loewii hoffmanni Rose et Schurian, 1977, Talysh

Samodurov, 2000: Plebejidea loewii ssp. loewii (Zeller, 1847), Armenian Highland

Samodurov, 2000: Plebejidea loewii ssp. schwingenschussii (Pfeiffer, 1937), Talysh Mts.

Tuzov, 2000b: Plebejidea loewii schwingenschussi Pfeiffer, Talysh Mts., Zuvand Plateau

Tschikolovets, Nekrutenko, 2012: Plebejus loewii loewii (Zeller, 1847), Nakhichevan, Daralagez Mts., Buzgov; Talysh, HiliDara; Galabyn; Alty-Agatsh

Korb, Bolshakov, 2016: Plebejus loewii (Zeller, 1847), Azerbaijan

\section{Kretania alcedo alcedo (Christoph, 1877)}

Tuzov, 1993: Cedoalia alcedo Chrustoph, 1877, Armenian plateau; Talysh

Nekrutenko, 1985a: Vacciniina alcedo (Christoph, 1877), Nakhichevan, Zangezur Mts., Bitshenek; Talysh, Zuvand, Lerik distr., Gosmolyan

Samodurov, 2000: Vacciniina alcedo(Christoph, 1877), Armenian Highland; Talysh

Zhdanko, 2000: Vacciniina alcedo madina ssp. n., Zangezurskyi Mts., Bichenek vill.

Tuzov, 2000b: Vacciniina alcedo alcedo Christoph. Talysh Mts., Zuvand Plateau

Tshikolovets, 2011: Plebejus alcedo alcedo (Christoph, 1877) (= madina Zhdanko, 2000), Talysh

Tschikolovets, Nekrutenko, 2012: Plebejus alcedo alcedo (Christoph, 1877), Ms.Talysh, Hili-Dara, Tatoni

Korb, Bolshakov, 2016: Kretania alcedo(Christoph, 1877), Azerbaijan

\section{Kretania eurypilus eurypilus (Freyer, [1851])}

Romanoff, 1884: Lycaena Eurypylus Frr., Ordoubad

Didmanidze, 1979: Plebejus eurypilus Frr., Turianchay Reservation; Shirvan steppe, Lake Adzhikabul; road to Baku; Nizovaya, Primorye

Tuzov, 1993: Kretania eurypilus iranica Forster, 1938, Armenian plateau; Talysh

Hesselbarth, van Oorschot \& Wagener, 1995: Plebeius (Kretania) eurypilus euripilus (Freyer, [1851]) (=Carmon Gerhard, [1851]), Alty Agatsch

Koçak, 1996: Polyommatus (Kretania) Carmon ssp. iranicus Forts., 1938, Azerbayejan

Samodurov, 2000: Kretania eurypilus eurypilus (Freyer, [1851]) (= Carmon Gerhard, [1851]; = iranica Forster, 1938), Armenian Highland; Talysh 
Tuzov, 2000b: Kretania eurypilus eurypilus Freyer, Talysh Mts., Zuvand Plateau

Tshikolovets, 2003: Plebejus eurypilus, Alty-Agatsh

Didmanidze, 2004: Plebejus eurypilus eurypilus Freyer, 1852, Turianchai Reservation, Niozvaya, Primorie, Shirvan steppe near Lake Ajicakub, near Baku

Tshikolovets, 2011: Plebejus eurypilus eurypilus (Freyer, [1851]), Alty-Agach

Tschikolovets, Nekrutenko, 2012: Plebejus eurypilus eurypilus (Freyer, [1851]), Nakhichevan, Daralagez Mts., Kyukyu; Talysh, Lerik distr., Gosmolyan; Hili-Dara; Kolvaz; Elisavetpol

Korb, Bolshakov, 2016: Kretania eurypilus (Freyer, 1852), Azerbaijan

Material: 10, Nakhichevan, Ordubad, Agdere 27.07.2018; 10, 19, Siyazan, Beshbarmak, 12.05.2015

132.Afarsia morgianus blomi (Rose \& Schurian, 1977)

Nekrutenko, 1985a: Vacciniina hyrcana (Lederer, 1870), Nakhichevan, Daralagez Mts., Buzgov

Korshunov \& Dubatolov, 1987: Vacciniina hyrcana Ld., Nakhichevan, Buzgov

Samodurov, 2000: Farsia morgiana (Kirby, 1871) (= hyrcana Lederer, [1869]), Armenian Highland

Tuzov, 2000b: Farsia morgiana morgiana Kurby, Nakhichevan, Buzgov

Weidenhoffer \& Klir, 2000: Plebejus (Vacciniina) morgianus, Nakhichevan, Daralagezsky Ridge, Buzgov

Ten Hagen \& Shurian, 2001: Plebejus (Vacciniina) morgianus blomi, Nakhichevan, Daralagezskiy khrebet, Buzgov)

Skala \& Weidenhoffer, 2002: Plebejus morgianus nakhichevanus Weidenhoffer et Skala, sp.nov., Nakhichevan, daralagezskyi Ridge, Buzgov

Didmanidze, 2004: Vacciniina hyrcanus Lederer, 1869: Nakhichevan, Belav, Kivrak

Tshikolovets, 2011: Plebejus morgianus blomi (Rose \& Schurian, 1977), Nakhichevan

Material: 19, Nakhichevan, Ordubad, Agdere, 28-30.07.2016

\section{Eumedonia eumedon eumedon (Esper, [1780])}

Effendi, 1970: Lycaena eumedon Esp., Shamakha, Pirkuli; Kutkashen (now Gabala), Chomcha-Bulag; Yardymly, Chayuzi Nekrutenko, 1972: Eumedonia eumedon modestus Nekrutenko, new subspecies, Yelizavetpol (now Kirovabad)

Eitschberger \& Steiniger, 1975: Eumedonia eumedon modestus Nekrutenko, 1972, Yelizavetpol (now Kirovobad)

Tuzov, 1993: Eumedonia eumedon eumedon Esper, 1780, Talysh

Koçak, 1996: Polyommatus (Eumedonia) eumedon ssp. modestus Nekr., 1972, Azerbayejan

Tuzov, 2000a: Eumedonia eumedon ssp. eumedon (Esoer, [1780]) (= Osiris O.Bang-Haas, 1927), Talysh

Korb, Bolshakov, 2016: Eumedonia eumedon (Esper, [1780]), Azerbaijan

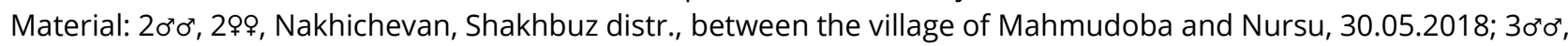
Astara, Toradi, 13-16.06.2016; 20 $0^{\circ}$, Lerik, Gosmalyan, 23.06.2017; 5m, 2 f, same place, 21-22.06.2017; 20" Nakhichevan,

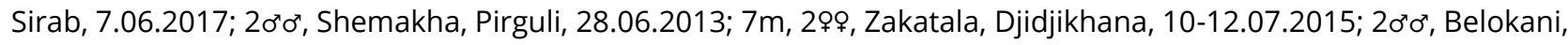
Beshbulag, Djidjikhana, 25.06.2013

134.Aricia agestis agestis ([Denis \& Schiffermüller], 1775)

Christoph, 1874: Lycaena Astrarche Bergstr., Lenkoran

Romanoff, 1884: Lycaena Astrarche Bgstr. et v. gen. II. Aestiva Stgr., Ordoubad

Christoph, 1886: Lycaena Astrarche Bgstr., Lenkoran, Talysh

Effendi, 1970: Lycaena astrarche Bhstr. (= medon Esp., = agestis Schiff., = nazira Moore), everywhere on them and in the foothills

Tuzov, 1993: Aricia agestis calida Bell., Armenian plateau; Talysh

Tuzov, 2000b: Aricia agestis azerbaidzhana Obraztsov, Talysh Mts., Lerik; Nakhichevan, Ordubad; Talysh Mts., Zuvand Plateau

Tschikolovets, Nekrutenko, 2012: Plebejus agestis agestis ([Denis \& Schiffermüller], 1775), Mingetshaur; Akstafa, Pojlu; Jenikend; Ms.Talysh, Kosmoljan; Talysh, Zuvand, Divagatsh; Adji-kent; Elisavetpol; Terter

Korb, Bolshakov, 2016: Aricia agestis ([Denis et Schiffermüller], 1775), Azerbaijan

Material: $20^{\pi} 0^{\top}$, Shemakha, Pirkuli, 28.06.2013.

135.Aricia anteros anteros (Freyer, [1838])

Effendi, 1970: Lycaena anteros Frr. ssp. crassipuncta Chr., Talysh, Yardimli, Lerik; Ordubad, Unus, Pazmara Nekrutenko, 1980: Aricia (Ultraaricia) anteros anteros (Freyer), Nakhichevan, Bitshenek (Mts. Zangezur)

Tuzov, 1993: Ultraaricia anteros anteros Freyer, 1893, Armenian plateau; Talysh

Koçak, 1996: Polyommatus (Ultraaricia) anteros ssp. anteros Frr., 1839, Azerbayejan

Bogdanov, 2000: Ultraaricia anteros (Freyer, [1839]), Armenian Highland

Tuzov, 2000b: Ultraaricia crassipuncta Christoph, Nakhichevan, Pass Bichenek

Tshikolovets, 2011: Plebejus anteros anteros (Freyer, [1838]), W.Azerbaijan

Tschikolovets, Nekrutenko, 2012: Plebejus anteros anteros (Freyer, [1838]), Nakhichevan, Ordubad vic.; Gandzha

Korb, Bolshakov, 2016: Aricia anteros (Freyer, 1839), Azerbaijan 


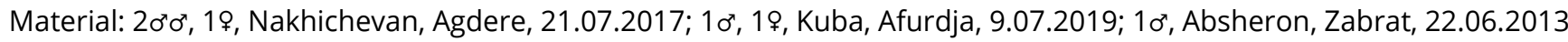

136. Aricia crassipunctus crassipunctus (Christoph, 1893)

Tuzov, 1993: Ultraaricia crassipuncta Christoph, 1893, Armenian plateau

Hesselbarth, van Ooeschor \& Wagener, 1995: Plebejos (Aricia) anteros crassipuncta (Christoph, 1893), Nachtshevan

Bogdanov, 2000: Ultraaricia crassipuncta (Christoph, 1893), Armenian Highland

Bogdanov, 2000: Ultraaricia crassipuncta crassipuncta (Christoph, 1893), Armenian Highland

137. Aricia vandarbani vandarbani(Pfeiffer, 1937)

Nekrutenko, 1980: Aricia (Ultraaricia) vandarbani(Pfeiffer), comb. et stat. nov., Mts.Talysh, Lerik

Tuzov, 1993: Ultraaricia vandarbani Pfeiffer, 1937, Talysh

Koçak, 1996: Polyommatus (Ultraaricia) vardarbanissp. vandarbani Pff., 1937, Azerbayejan

Bogdanov, 2000: Ultraaricia vandarbani(Pfeiffer, 1937), Talysh Mts.

Tuzov, 2000b: Ultraaricia vandarbani Pfeiffer, Talysh Mts., Lerik

S.K.Korb, 2005: Aricia vandarbani vandarbani Pfeiffer, 1937, Talysh

Korb, Bolshakov, 2016: Aricia vandarbani(Pfeiffer, 1937), Azerbaijan

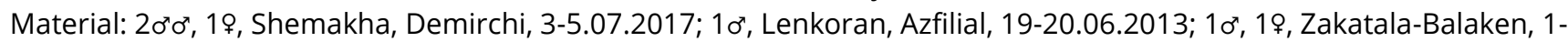
4.04.2014; 19, Nakhichevan, Ordubad, Agdere, 11.06.2017; 10', 19, Lenkoran, Azfilial, 16-19.09.2013; 20 $0^{\prime \prime}$, 19, same place, 26-28.05.2014; 20 $0^{\prime \prime}$, 19, Ismailly Kaladjik, Velasin, 21-23.07.2014; 20 $0^{\prime \prime}$, Massali, near Kubin and Mijankyu vill., 5.05.2014; $20^{\circ} 0^{\prime \prime}, 2 \% \circ$, Kuba, Afurdja, 8.07,2019; 20"0', 1\%, Absheron, Zabrat, 22.06.2013

138. Agriades pyrenaicus dardanus (Freyer, [1844])

Romanoff, 1884: Lycaena Orbitulus Prun. var. Dardanus Frr., Helenendorf

Effendi, 1970: Lycaena orbitulus Prun. ssp. dardanus Frr., Shakhadag Mt.

Tuzov, 1993: Agriades pyrenaicus araraticus Gerhard, 1853, Armenial plateau

Tuzov, 2000a: Agriades pyrenaicus ssp. dardanus (Freyer, [1844]) (= araraticus Gerhard, 1853; = latedisjunctus Alberti, 1973), Armenian Highland

Tuzov, 2000b: Agriades pyrenaicus ssp. dardanus Freyer, Nakhichevan, Buzgov near Ordubad

Tschikolovets, Nekrutenko, 2012: Plebejus pyrenaicus dardanus (Freyer, [1844]), Ms. Murovdagh (gub. Elisavetpol)

Korb, Bolshakov, 2016: Agriades pyrenaicus (Boisduval, 1840), Azerbaijan

Material: 10, Nakhichevan, Shakhbuz, Bichenek, Shakhbuz National Park, 26.07.2016

139. Neolysandra coelestinus alticola (Christoph, 1893)

Didmanidze, 1979: Albulina chrisopis Gr.-Gr., Nakhichevan, Nakhichevanchai River gorge, Arindj; Paragachai river gorge, Bilav; Kapudjik ridge

Nekrutenko, 1983: Neolysandra alticola (Christoph, 1893), comb. et stat. n. = Albulina alexander Higgins, 1981, syn. n., Azerbaijan

Tuzov, 1993: Neolysandra coelestinus alticola Christoph, 1886, Armenian plateau

Tuzov, 1993: Neolysandra alexander Higgins, 1981, Armenian plateau

Tuzov, 2000a: Neolysandra coelestina ssp. alticola (Christoph, 1886), Armenian Highland

Tuzov, 2000b: Neolysandra coelestina alticola Christoph, Nakhichevan, Buzgov

Didmanidze, 2004: Albulina chrysops Grum.Grshimailo, 1888, Nakhichevan, Paragachai gorge, Bilav

Tshikolovets, 2011: Polyommatus coelestinus alticola (Christoph, 1893) (= alexander Higgins, 1981), Nakhichevan Tschikolovets, Nekrutenko, 2012: Polyommatus coelestinus alticola (Christoph, 1893), Nachitshevan, Paragarshaj fluv.val. Korb, Bolshakov, 2016: Polyommatus coelestinus (Eversmann, 1843), Azerbaijan

Material: 10, Nakhichevan, Shakhbuz, Nursu, 8.06.2017

140.Neolysandra diana diana (Miller, 1913)

Effendi, 1970: Lycaena diana Mill., Nakhichevan, Paiz

Tuzov, 1993: Neolysandra diana Miller, 1913, Armenian plateau

Tuzov, 2000d: Neolysandra diana (Miller, [1913]), Armenian Highland

141. Cyaniris semiargus bellis (Freyer, [1842])

Christoph, 1886: Lycaena semiargus Rott., Talysch

Effendi, 1970: Lycaena semiargus Rott. ssp. bellis Frr., Aterk, Agdavan, Yanshakh, Goygol Lake; neighborhood of Shakhdag and Babadag Mts.

Didmanidze, 1979: Aricia semiargus Rott., Nakhichevan, Shakhbuz ridge

Tuzov, 1993: Cyaniris bellis intermedia Tutt, 1909, Armenian plateau; Talysh

Tuzov, 2000a: Cyaniris bellis ssp. antiochena (Lederer, 1861), Armenian Highland; Talysh Mts.

Didmanidze, 2004: Cyaniris semiargus Rottemburg, 1775, Turianchai Reservation; Nakhichevan; Mount Shakhbuz 
Tschikolovets, Nekrutenko, 2012: Polyommatus semiargus bellis (Freyer, [1842]), Elisatpol; Alty-Agatsh; Talysh-Zuvand, Hili Dara

Korb, Bolshakov, 2016: Cyaniris semiargus (Rottemburg, 1775), Azerbaijan

Material: $1 \sigma^{\top}$ Kedabek, Novoivanovka -Novosaratovka vill., 19-21.06.2014

142. Polyommatus thersites thersites (Cantener, [1835])

Didmanidze, 1979: Lycaena thersites Caut., Nakhichevan, Paragachay river gorge, Bilav

Tuzov, 1993: Polyommatus thersites ketshevana Obraztsov, 1936, Armenian plateau; Talysh

Koçak, 1996: Polyommatus (Plebicula) thersites ssp. rjabovi Obr., 1936, Azerbayejan

Tuzov, Zhdanko \& Dantchenko, 2000: Polyommatus thersites ssp. thersites (Canterer, 1834) (= narzana Obraztsov, 1936; = gandzhana Obraztsov, 1936; = karatshaica Obraztsov, 1936; = rjabovi Obraztsov, 1936), Armenian Highland; Talysh Mts.

Didmanidze, 2004: Polyommatus thersites Cantener, 1834, Turianchai reservation

Tschikolovets, Nekrutenko, 2012: Polyommatus thersites thersites (Cantener, [1835]), Talysh, Zuvand

Korb, Bolshakov, 2016: Polyommatus thersites (Cantener, 1834), Azerbaijan

Material: 20'0', Shemakha, Pyrguli, 25.06.2015

\section{Polyommatus myrrha bakkariensis (Koçak, 1977)}

Effendi, 1970: Lycaena myrrha HS. ssp. myrrhina Stgr., Talysh, Zuvand, Kosmolyan

Korshunov, 1972: Polyommatus myrrhus Herrich-Schäffer, 1851, Zangezur Mts., Bitshenek pass.

Nekrutenko \& Effendi, 1979: Lysandra cinyrea Nekrutenko et Effendi sp. nov., Nakhichevan, Daralagezsky distr., Kyukyu village

Tuzov, 1993: Sublysandra myrrha latimargo Courvoisier, 1913, Armenian plateau

Koçak, 1996: Polyommatus (Sublysandra) myrrhus ssp. cinyraeus Nekr. \& Eff., 1979, Azerbayejan

Tuzov, 2000b: Polyommatus mirrha cinyrea Nekrutenko et Effendi, Nakhichevan, Kyukyu

Tshikolovets, 2011: Polyommatus myrrha bakkariensis (Koçak, 1977), Nakhichevan, Kyukyu

144. Polyommatus dorylas armenus (Staudinger, 1871)

Effendi, 1970: Lycaena hylas Esp. ssp. armena Stgr., Nakhichevan, Paiz; Lerik, Kosmolyan, Kyalyakhan

Tuzov, 1993: Plebicula dorylas armena Staudinger, 1871, Armenian plateau

Tuzov, Zhdanko \& Dantchenko, 2000: Poluommatus dorylas ssp. armenus Staudinger, 1871, Armenian Highland

Zhdanko, 2002: Polyommatus dorylas ssp. armena (Staudinger, 1871), Armenian Highland

Tschikolovets, Nekrutenko, 2012: Polyommatus dorylas armenus (Staudinger, 1871), Amamly (?)

\section{Polyommatus amandus amandus (Schneider, 1792)}

Effendi, 1970: Lycaena amanda Schn. ssp. orientalis Stgr., everywhere in forest foothills and subalpine meadows Didmanidze, 1979: Lycaena amandus Schn., road to Baku; Nakhichevan, Paragachay River gorge, Bilav; Shakhbus ridge Tuzov, 1993: Plebicula amandus orientalis Staudinger, 1901, Armenian plateau; Talysh

Didmanidze, 2004: Polyommatus amandus Scheider, 1792, near Baku; Nakhichevan, Paragachai gorge, Bilav

Tschikolovets, Nekrutenko, 2012: Polyommatus amandus amandus (Schneider, 1792), Nkhcihevan, Ordubad, Alakhi;

Traj.Bitshenek; Bilav, Paragatshai fl. val.; Alty-Agatsh

Korb, Bolshakov, 2016: Polyommatus amandus (Schneider, 1792), Azerbaijan

Material: $1 \sigma^{\prime \prime}$, Nakhichevan, Ordubad, Agdere 23-24.07.2018; 30'0", Shakhbuz, Nursu, 8.06.2017

146. Polyommatus bellargus bellargus (Rottenburg, 1775)

Ménétriés, 1832: Polyommatus Adonis, Fab., Lencoran

Lederer, 1870: Lycaena Adonia S. V., Lenkoran

Effendi, 1970: Lycaena bellargus Rott., everywhere in the foothills, at least in the lowlands and in the mountains

Didmanidze, 1979: Lycaena bellargus Rott., Turianchay Reserve; Nakhichevan, Bilav

Tuzov, 1993: Lysandra bellargus Rottemburg, 1775, Armenian plateau; Talysh

Tschikolovets, Nekrutenko, 2012: Polyommatus bellargus bellargus (Rottenburg, 1775), Zangezur Mts., Bitshenek; Talysh, Bitshegan pass.; Bilav, Paraga-cai fluv., val.; Mts. Talysh, Hili-Dara, Galabin; Adzh-Kent; Elizavetpol

Korb, Bolshakov, 2016: Polyommatus bellargus (Rottemburg, 1775), Azerbaijan

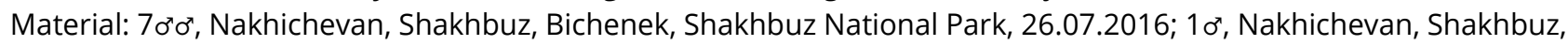
Kyukyu, 27.07.2016; 20 $0^{\circ}$, 19, Nakhichevan, Ordubad, Agdere 23-24.07.2018; $10^{\prime \prime}, 19$, same place, 9-12.06.2017; 5\%9,

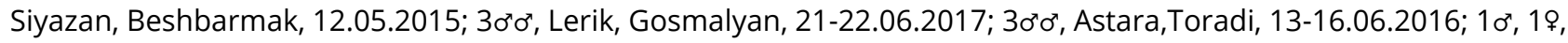
Apsheron, near Zoologican Institute, 10.05.2013

147.Lysandra corydonius corydonius (Herrich- Schäffer, [1852])

Romanoff, 1884: Lycaena Coridon Pod aver. Caucasica Ld., Hankynda 
Effendi, 1970: Lycaena corydon Poda ssp. caucasica Ld., everywhere in the foothills, rarely on lowlands, extremely rare in the mountains

Schurian, 1989: Polyommatus (Lysandra) corydonius corydonius Herrich-Scäffer, 1852, Helenendorf

Tuzov, 1993: Lysandra ciscaucasicus Jachontov, 1914, Armenian plateau; Talysh

Dantchenko, 2000: Lysandra corydonius ssp. caucasica (Lederer, [1870]), Armenian Highland; Nakhichevan, Talysh Mts.

Dantchenko, 2000: Lysandra sheikh Dantchenko, spec. nov., Kusary River valley

Tuzov, 2000b: Lysandra corydonius corydonius Herrich- Scäffer, Kirovobad (Gäncä), Kamo

Tuzov, 2000b: Lysandra corydonius caucasica Lederer, Nakhichevan, Buzgov

Tschikolovets, Nekrutenko, 2012: Polyommatus corrydonius corydonius (Herrich- Schäffer, [1852]), Nachitshevan, pag. Bitshenek; Mts. Talysh, Hili-Dara, Kosmolyan; Kirovobad (Gäncä), Kamo

Korb, Bolshakov, 2016: Polyommatus corydonius (Herrich-Schäffer, [1851]), Azerbaijan

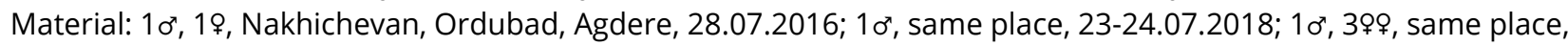

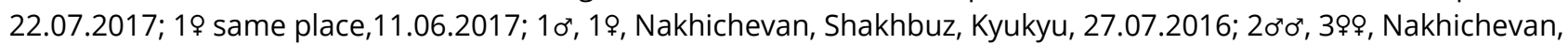
Shakhbuz near Makhmudoba and Nursu, 30.05.2018; 10", 19, Shemakha, Archiiman-Pirguli, near Shakhdag Milli Park,1920.05.2013; 10", Belokani, Beshbulag, Djidjikhana, 25.06.2013; 30 $0^{7}$, Ismailly Kaladjik, Velasin, 21-23.07.2014

148. Polyommatus (Meleageria) daphnis daphnis ([Denis \& Schiffermüller], 1775)

Tshikolovets, 2003: Polyommatus daphnis, Alty-Agatsh

Tshikolovets, 2011: Polyommatus daphnis daphnis ([Schiffermüller], 1755), Alty-Agatsh

Korb, Bolshakov, 2016: Polyommatus daphnis ([Denis et Schiffermüller], 1775), Azerbaijan

149. Polyommatus (Meleageria) daphnis versicolor (Heyne, [1895])

Ménétriés, 1832: Polyommatus Meleager Fab., Talyche

Lederer, 1870: Lycaena Daphnis S. V. et var. S tevenii Hb, Talyche

Romanoff, 1884: Lycaena meleager Esp., var. Steveni Tr., Hankynda

Tuzov, 1993: Meleageria daphnis dugijani Schirian et Hauser, 1981, Armenian plateau; Talysh

Effendi, 1970: Lycaena meleager Esp., Shemakha, Kirovka; Konakhkend, Ryuk; Aterk; Ordubad; Talysh, Kosmolyan Tuzov, 2000a: Meleageria daphnis ssp. elamita (Le Cerf, 1913), Talysh

Tuzov, 2000a: Meleageria daphnis versicolor (Heyne, [1895]), Armenian Highland

Tuzov, 2000b: Meleageria daphnis versicolor Heyne, Nakhichevan, Buzgov

Tuzov, 2000b: Meleageria daphnis ssp. elamita Le Cerf, Talysh Mts., Zuvand Plateau

Tschikolovets, Nekrutenko, 2012: Polyommatus daphnis versicolor (Heyne, [1895]), Adzhi-kent; Nakhichevan, Bitshenek pass; Ordubad, Alakhi vill., Traj. Bitshenek

Korb, Bolshakov, 2016: Polyommatus daphnis ([Denis et Schiffermüller], 1775), Azerbaijan

150. Polyommatus (Polyommatus) marcidus marcidus (Lederer, 1870)

Tuzov, 1993: Meleageria marcida Lederer, 1871, Talysh

Tuzov, 2000b: Meleageria daphnis elamita Le Cerf f. marcida Lederer, Talysh Mts., Zuvand Plateau

S.K.Korb, 2005: Meleageria marcida marcida Lederer, 1871, Talysh

Tschikolovets, Nekrutenko, 2012: Polyommatus marcidus marcidus (Lederer, 1870), Ms.Talysh-Zuvand; Kosmoljan, HiliDara, Golabyn; Lerik, Juchary Ambur-dara; Kishlak vill., Allar

151.Polyommatus (Polyommatus) icarus icarus (Rottemburg, 1775)

Christoph, 1877: Lycaena Icarus Rott., Baku

Christoph, 1886: Lycaena Icarus Rott., Lenkoran

Rühl, [1893]: Lycaena Icarus Rott., Baku

Effendi, 1970: Lycaena icarus Rott. ssp. icarinus Scriba, everywhere to the alpine zone

Didmanidze, 1979: Polyommatus icarus Rott., Baku; Mil steppe, lake Aggel; Turianchai Reservation; Mil steppe, Saatly environs; Sumgait; Gobustan; Tourogai ridge; road to Baku, pass; Nakhichevan, Paragachay railway; Bilav; Ordubad environs, Paragachay river gorge, Kapujuh Ridge; the environs of Nakhichevan; Shakhbuz Ridge

Tuzov, 1993: Polyommatus icarus icarus Rottemburg, 1775, Armenian plateau; Talysh

Tschikolovets, Nekrutenko, 2012: Polyommatus icarus Icarus (Rottemburg, 1775), Samedabad distr., Muganskaya steppe; Talysh, Gosmolyan; Shuvi vill.; Kindzhaba; Talysh, geran vill.; Ordubad; Talysh, Zuvand Divagatsh, Gosmoljan; Mts. Talysh, Lerik; Jenikend; Akstafa vic., Pojlu; Baku, Nagorny park

Korb, Bolshakov, 2016: Polyommatus icarus (Rottemburg, 1775), Azerbaijan

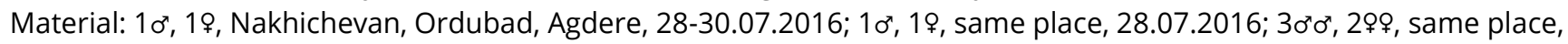

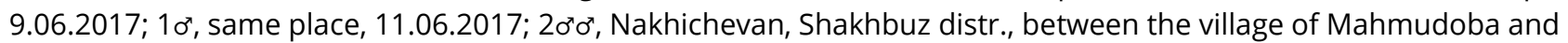
Nursu, 30.05.2018; 10 1\%, Nakhiichevan, Shakhbuz, Nursu, 15.06.2019; 50" $0^{\prime \prime}$, Siyazan, Beshbarmak, 12.05.2015; 10", Shemakha, Demirchi, 3-5.07.2017; Kedabek, Novosaratovka vill., 6-7.10.2015; 50"0', Yardimli,17-20.05.2016; 50"0', 39o Kedabek, Novoivanovka -Novosaratovka vill., 19-21.06.2014; 20º', Shakhbuz, Nursu, 8.06.2017; 10, 1\%, Lenkoran, 


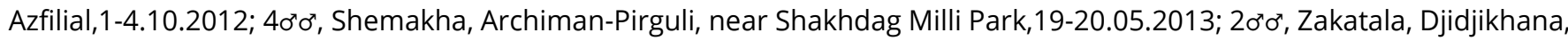
10-12.07.2015; 20' $0^{\prime \prime}$, Zakatala, from kombinat to State Reserve, 24.06.2014; 30" $0^{\prime \prime}$, Apsheron, near Zoologican Institute,

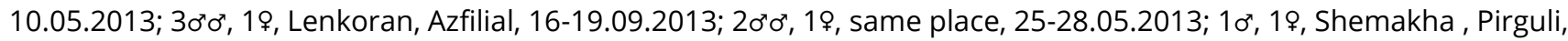
08.2013; 20'0", Ismailly Kaladjik, Velasin, 21-23.07.2014

\section{Polyommatus (Polyommatus) eros erotulus Nekrutenko, 1985}

Effendi, 1970: Lyacena eros O. ssp. eroides Frin., the southern slopes of the Mrovdag and Mykhtekan (Mıxtəkən) ranges Nekrutenko, 1985b: Polyommatus eroides erotulus Nekrutenko, ssp.n., Talysh, Zuvand, Mystan vill.

Bálint, [1993]: Polyommatus forsteri erotulus (Nekrutenko, 1985), comb. n., Azerbaidzhan

Tuzov, 1993: Polyommatus eros erotulus Nekrutenko, 1985, Talysh

Carbonell, 1994: Polyommatus erotulus Nekrutenko, 1985 n. stat. Talysch

Koçak, 1996: Polyommatus (s. str.) erotulus ssp. erotulus Nekr., 1985, Azerbayejan

Tuzov, Zhdanko \& Dantchenko, 2000: Polyommatus erotulus erotulus Nekrutenko, 1985 (= delessei Carbonel, 1994),

Talysh Mts.

Tuzov, 2000b: Polyommatus erotulus Nekrutenko, Talysh Mts., Mistan Voodolazhsky \& Stradomsky, 2008: Polyommatus eros erotulus Nekrutenko, 1985, Talysh

Talysh, Zuvand, Mistan village environs

\section{Polyommatus (Agrodiaetus) ripartii paralcestis (Forster, 1960)}

Lederer, 1864: Lycaena Rippertii B., Helenendorf bei Elisabethpol

Tschikolovets, Nekrutenko, 2012: Polyommatus ripartii paralcestis (Forster, 1960), Talysh; Nakhichevan, Daralagez,

Azizbekov; Jelisavetpol

Korb, Bolshakov, 2016: Polyommatus ripartii (Freyer, 1830), Azerbaijan

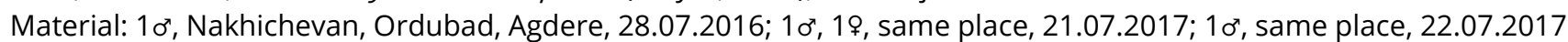

154.Polyommatus (Agrodiaetus) demavendi demavendi(Pfeiffer, 1938)

Tuzov, 1993: Agrodiaetus demavendi demavendi Pfeiffer, 1938, Talysh

Dantchenko, 2000b: Agrodiaetus demavendi demavendi (Pfeiffer, 1938), Armenian Highland; Talysh

Tuzov, 2000b: Agrodiaetus demavendi demavendi Pfeiffer, Talysh Mts., Zuvand Plateau, Gosmalyan

Tshikolovets, 2003: Agrodiaetus demavendi, Alty-Agatsh

S.K.Korb, 2005: Polyommatus demavendi demavendi (Pfeiffer, 1938), Talysh

Tshikolovets, 2011: Polyommatus demavendi (Pfeiffer, 1938), Alty-Agach

Tschikolovets, Nekrutenko, 2012: Polyommatus demavendi demavendi (Pfeiffer, 1938), Lerik, Talysh, Hili-Dara, Divagatsh (Divağaç), Galabin (Gələbin); Jelizavetpol; Nakhichevan, Buzgov

Korb, Bolshakov, 2016: Polyommatus demavendi (Pfeiffer, 1938), Azerbaijan

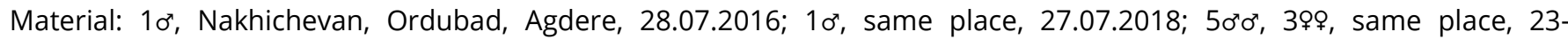
24.07.2018

155. Polyommatus (Agrodiaetus) eriwanensis eriwanensis (Forster, 1960)

Forster, 1960: Argodiaetus ripartii eriwanensis ssp. nov., Nachitshevan, Mts. Daralagez, Germatshatach; Nus-Nus pr.Ordubad; Jelisavetpol

Tuzov, 1993: Argodiaetus demavensi erivanensis Fortser, 1960, Armenian Plateau

Tshikolovets, 2011: Polyommatus eriwanensis (Forster, 1960), W. Azerbaijan

Tschikolovets, Nekrutenko, 2012: Polyommatus eriwanensis eriwanensis (Forster, 1960), Traj.Bitshenek; Adzhi-Kent (prope Jelisavetpol); Nakhichevan, mts. Daralagez, Germatshatach

Korb, Bolshakov, 2016: Polyommatus eriwanensis (Forster, 1960), Azerbaijan

159. Polyommatus (Agrodiaetus) admetus malievi (Dantchenko et Lukhtanov, 2004)

Effendi, 1970: Lycaena admetus Esp. ssp. ripartii Frr., Nakhichevan, Paiz; Talysh, Zuvand

Dantchenko \& Lukhtanov, 2004: Agrodiaetus admetus malievi subspec. nov., Talysh Mts., Zuvand Plateau, Mistan vill., Lyulyakeran village

Vila, Lukhtanov, Talaverta, Gil-T. \& Pierce, 2010: Agrodiaetus admetus malievi, Talysh, Zuvand

Tschikolovets, Nekrutenko, 2012: Polyommatus admetus admetus (Esper, [1783]), Jelisavetpol

Lukhtanov et al., 2015: Agrodiaetus admetus malievi (Dantchenko et Lukhtanov, 2004)

Korb, Bolshakov, 2016: Polyommatus malievi (Dantchenko et Lukhtanov, 2004), Azerbaijan, Talysh mts.

160. Polyommatus (Agrodiaetus) rjabovianus rjabovianus Koçak, 1980

Forster, 1960: Agrodiaetus rjabovisp. nov., Talysh distr., Lenkoran, Ljulakeran; Zuvant, ms. Kyzjurdy

Korshunov, 1972: Polyommatus rjabovi Forster, 1960, Talysh

Tuzov, 1993: Agrodiaetus rjabovi rjabovi Forster, 1960, Talysh

Dantchenko, 2000: Agrodiaetus rjabovi rjabovi Forster, Zuvand Plateau, Talysh Mts. 
Tuzov, 2000b: Agrodiaetus rjabovi rjabovi Forster, Talysh Mts., Zuvand Plateau; Talysh Mts., Veri S.K.Korb, 2005: Polyommatus rjabovi rjabovi (Forster, 1960), Talysh

Vila, Lukhtanov, Talaverta, Gil-T. \& Pierce, 2010: Agrodiaetus rjabovi, Talysh, Zuvand

Tschikolovets, Nekrutenko, 2012: Polyommatus rjabovi rjabovi (Forster, 1960), Talysh, Zuvand, Mistan; Talysh, Allar Lukhtanov et al., 2015: Polyommatus rjabovianus rjabovianus Koçak, 1980: Talysh Mts.

Korb, Bolshakov, 2016: Polyommatus valiabadi rjabovianus Koçak, 1980: Talysh, Lenkoran

161. Polyommatus (Agrodiaetus) pseudorjabovi Lukhtanov, Dantchenko, Vishnevskaya et Saifitdinova, 2015

Lukhtanov et al., 2015: Polyommatus (Agrodiaetus) pseudorjabovi. Azerbaijan, Talysh, Zuvand

Korb, Bolshakov, 2016: Polyommatus (Agrodiaetus) pseudorjabovi. Azerbaijan, Talysh, Zuvand

\section{Polyommatus (Agrodiaetus) ninae ninae (Forster, 1956)}

Forster, 1956: Agrodiaetus transcaspica ninae ssp. nov., Nachitshevan, mts. Daralagez, Germatshatach

Tuzov, 1993: Agrodiaetus ninae ninae Forster, 1956, Armeniam plateau

Hesselbarth, van Oorschot \& Wagener, 1995: Polyommatus (Agrodiaetus) ninae (Forster, 1956), Nachitschewan

Dantchenko, 2000: Agrodiaetus ninae Forster, 1956, Armenian Highland

Tschikolovets, Nekrutenko, 2012: Polyommatus ninae ninae (Forster, 1956), Nakhichevan, Daralagez, Buzgov; Ordubad distr., Pazmara; Zangezur Mts., Bitshenek

162. Polyommatus (Agrodiaetus) aserbeidschanus aserbeidschanus (Forster, 1956)

Forster, 1956: Agrodiaetus transcaspica aserbeidschana ssp. nov., Talysh, Zuvant, mts. Kyz-jurdy (Gızyurdu) (distr.Lenkoran)

Lukhtanov, 1989: Agrodiaetus aserbeidschana Forster, 1956, Talysh, Lerik distr., Galobin

Tuzov, 1993: Agrodiaetus aserbeidschana Forster, 1956, Talysh

Koçak, 1996: Polyommatus (Argodiaetus) aserbeidschanus ssp. aserbeidschanus Forst., 1956, Azerbayejan

Eckweiler \& Häuser, 1997: Polyommatus (Argodiaetus) transcaspicus aserbeidschanus, Talysh, Zuvand

Dantchenko, 2000: Agrodiaetus aserbeidschana Forster, 1956, Armenian Highland; Talysh Mts. (Zuvand Plateau)

Tuzov, 2000b: Agrodiaetus aserbeidschanus aserbeidschanus Forster, Talysh Mts., Zuvand Plateau

Eckweiler, 2002: Polyommatus (Argodiaetus) aserbeidschanus, Talysh, Zuvand, Mystan

Tshikolovets, 2003: Polyommatus aserbeidschanus, Talysh, Gosmolyan

Tshikolovets, 2011: Polyommatus aserbeidschanus (Forster, 1956), Talysh, Galabyn

Korb, Bolshakov, 2016: Polyommatus aserbeidschanus (Forster, 1956), Azerbaijan

163. Polyommatus (Agrodiaetus) lukhtanovi lukhtanovi(Dantchenko, 2004)

Dantchenko, 2000: Agrodiaetus aserbeidschanus Forster, 1956, Talysh Mts., Zuvand Plateau

Dantchenko, 2004: Agrodiaetus lukhtanovispec. nov., Talysh mts., Zuvand plateau, Mistan village; Galabin village Tshikolovets, 2011: Polyommatus lukhtanovi (Dantchenko, 2004), Talysh, Galabin, Hilia-Dara; Nakhichevan, Buzgov Korb, Bolshakov, 2016: Polyommatus lukhtanovi Dantchenko, 2004, Azerbaijan

164. Polyommatus (Agrodiaetus) damonides damonides (Staudinger, 1899)

Staudinger, 1899: Lycaena Damone Ev. var. Damonides, Ordubad

Forstr, 1961: Agrodiaetus Poseidon damonides Stgr., Nachitshevan, Ordubad

Effendi, 1970: Lycaena poseidon HS ssp. caerulea Stgr. , Norashen, Velidag (Vəlidağ) Mt., Kechaltala (Keçəltala) Mt.,

Ordubad, Paraga

Eckweiler \& Häuser, 1997: Polyommatus (Agrodiaetus) transcaspicus damonides, Ordubad

Tuzov, 1993: Agrodiaetus damonides Staudinger, 1899, Armenian plateau (Zangezursky Mts.)

Koçak, 1996: Polyommatus (Agrodiaetus) poseidon ssp. damonides Stgr., 1899, Azerbayejan

Olivier, Puplesiene, van der Poorten, De Prins \& Wiemers, 1999: Polyommatus (Agrodiaetus) damonides (Staudinger, 1899), Ordubad

Dantchenko, 2000: Agrodiaetus damonides (Staudinger, 1899), S.Armenian Highland

Tuzov, 2000b: Agrodiaetus damonides Staudinger, Nakhichevan, Nyusnyus

Tschikolovets, Nekrutenko, 2012: Polyommatus damonides damonides (Staudinger, 1899), Buzgov; Talysh, Zuvand

Korb, Bolshakov, 2016: Polyommatus damonides (Staudinger, 1899), Azerbaijan

165. Polyommatus (Agrodiaetus) surakovi surakovi Dantchenko \& Lukhtanov, 1994

Tuzov, 1993: Agrodiaetus carmon surakovi Dantchenko et Lukhtanov, 1994, Armenian pkateau (Daralagezsky Mts.) Dantchenko \& Lukhtanov, 1994: Polyommatus (Agrodiaetus) carmon surakovi subspec. nov., Nakhichevan, Buzgov Koçak, 1996: Polyommatus (Agrodiaetus) carmon ssp. surakovi Dantchenko et Lukhtanov, 1994, Nahcivan Tuzov, 2000b: Agrodiaetus surakovi Dantchenko et Lukhtanov, Nakhichevan, Buzgov 
Carbnell, Borie \& J.De Prins, 2004: Polyommatus (Agrodiaetus) surakovi Dantchenko et Lukhtanov, 1994, Nakhichevan, Daralagez, Buzgov

Tshikolovets, 2011: Polyommatus surakovi Dantchenko \& Lukhtanov, 1994, S.Azerbaijan

Tschikolovets, Nekrutenko, 2012: Polyommatus surakovi surakovi Dantchenko \& Lukhtanov, 1994, Talysh, Zuvand, Galabin

Korb, Bolshakov, 2016: Polyommatus carmon surakovi Dantchenko et Lukhtanov, 1994, Nakhichevan, Buzgov

166. Polyommatus (Agrodiaetus) huberti huberti Carbonell, 1993

Hesselbarth, van Oorschot \& Wagener, 1995: Polyommatus (Agrodiaetus huberti (Carbonell, 1993), Nachitschewan, Ordubad

Tuzov, 2000b: Agrodiaetus huberti Carbonell, Nakhichevan, Buzgov

167. Polyommatus (Agrodiaetus) damon kotshubeji (Sovinsky, 1915)

Ménétriés, 1832: Polyommatus Damon, Fab., Lenkoran

Kolenati, 1846: Lycaena Damon Fabr., in montibus Talyschensibus

Romanoff, 1884: Lycaena Damon Schiff., Helenendorf; Hankynda

Christoph, 1886: Lycaena Damon F., Lenkoran

Rühl, [1893]: Lycaena Damon Schiff, Hankynda

Effendi, 1970: Lycaena damon Schiff. ssp. natiokotshubeji Sov., everywhere in the mountains and foothills

Didmanidze, 1979: Agrodiaetus damone Ev. sbsp. wagneri Forst., Nakhichevan, Ordubad

Tshikolovets, 2011: Polyommatus damon kotshubeji (Sovinsky, 1915), S.Azerbaijan

Tschikolovets, Nekrutenko, 2012: Polyommatus damon kotshubeji (Sovinsky, 1915), Traj.Bitshinek

Korb, Bolshakov, 2016: Polyommatus damon ([Denis et Schiffermüller], 1775), Azerbaijan

168. Polyommatus (Agrodiaetus) iphigenia iphigenia (Herrich- Schäffer, [1847])

Romanoff, 1884: Lycaena Kindermanni Led. var. Iphigenia HS., Ordoubad

Effendi, 1970: Lycaena kindermanni Ld. ssp. iphigenia HS, Нахичевань, Паиз; Ордубад; Талыш, Лерик, Зуванд

Tuzov, 1993: Agrodiaetus iphigenia araratensis de Lesse, 1957, Armenian plateau (Zangezursky Mts.)

Tshikolovets, 2011: Polyommatus iphigenia iphigenia (Herrich- Schäffer, [1847]), Nakhichevan

Tschikolovets, Nekrutenko, 2012: Polyommatus iphigenia iphigenia (Herrich- Schäffer, [1847]), Nakhichevan, Daralagez Mts., Kyukyu

169. Polyommatus (Agrodiaetus) baytopi rovshani Dantchenko \& Lukhtanov, 1994

Dantchenko \& Lukhtanov, 1994: Polyommatus (Agrodiaetus) rovshanispec. nov., Talysh Mts., Zuvand, Mistan Koçak, 1996: Polyommatus (Agrodiaetus) rovshanissp. rovshani Dantchenko \& Lukhtanov, 1994, Talysh

Dantchenko, 2000: Agrodiaetus rovshani(Dantchenko \& Lukhtanov, 1994), Talysh Mts.; Zuvand plateau, Talysh Mts.

Tuzov, 2000b: Agrodiaetus rovshani Dantchenko et Lukhtanov, 1994, Talysh Mts., Zuvand Distr., Mistan

Korb, Bolshakov, 2016: Polyommatus baytopi rovshani Dantchenko \& Lukhtanov, 1994, Azerbaijan, Talysh

170. Polyommatus (Agrodiaetus) phyllis sheljuzhkoi (Forter, 1960)

Forster, 1960: Agrodiaetus phyllus vanensis de Lesse, Nachitshevan, Paratshaj

Effendi, 1970: Lycaena phyllis Chr., Nakhichevan, Payiz, Kyarmachatakh

Tuzov, 1993: Agrodiaetus phyllis sheljuzhkoi Forster, 1956, Armenian plateau; Talysh

Hesselbarth, van oorshot \& Wagener, 1995: Polyommatus (Agrodiaetus phyllus vanensis (de Lesse, 1957) (= sheljuzhkoi

Forster, 1960), Nachitschewan

Eckweiler \& ten Hagen, 1998: Polyommatus (Agrodiaetus) phyllus subsp. sheljuzhkoi (Forster, 1960), Nachichevan

Dantchenko, 2000: Agrodiaetus vanensis ssp. sheljuzhkoi Forster, 1960, Armenian Highland; Talysh Mts.

Tuzov, 2000b: Agrodiaetus vanensis sheljuzhkoi Forster, Talysh Mts., Zuvand Plateau

Tshiklovets, 2011: Polyommatus phyllis sheljuzhkoi (Forter, 1960), S.Azerbaijan

Tschikolovets, Nekrutenko, 2012: Polyommatus phyllis sheljuzhkoi (Forter, 1960), Talysh, Hili-Dara, Rozanov;

Nakhichevan, Daralagezsky Mts., Buzgov, Zangezursky Mts., Bitshenek; Nakhichevan, Nyus-Nyus; Talysh, Zuvand

Korb, Bolshakov, 2016: Polyommatus phyllis (Christoph, 1877), Azerbaijan

\section{Polyommatus (Agrodiaetus) phyllis nekrutenkoi Dantchenko \& Lukhtanov, 1994}

Dantchenko \& Lukhtanov, 1994: Polyommatus (Agrodiaetus) phyllis nekrutenkoi subspec. nov., mt.Diubrar Koçak, 1996: Polyommatus (Agrodiaetus) phyllis nekrutenkoi Dantchenko \& Lukhtanov, 1994, Azerbayejan

Dantchenko, 2000b: Agrodiaetus phyllis ssp. nekrutenkoi (Dantchenko \& Lukhtanov, 1994), NE Azerbaijan

Tuzov, 2000e: Agrodiaetus phyllis nekrutenkoi Dantchenko \& Lukhtanov, Dyubrar; Alty-Agach

Tshikolovets, 2003: Polyommatus phyllis nekrutenkoi, Mt. Dyubrar

Tshikolovets, 2011: Polyommatus phyllis nekrutenkoi, Mt. Diubrar 
Tschikolovets, Nekrutenko, 2012: Polyommatus phyllis nekrutenkoi Dantchenko \& Lukhtanov, 1994, Nakhichevan Mts., Bitshenek

Korb, Bolshakov, 2016: Polyommatus phyllis nekrutenkoi Dantchenko \& Lukhtanov, 1994, Azerbaijan, East Caucasus, mt. Diubrar

172. Polyommatus (Agrodiaetus) altivagans altivagans (Forster, 1956)

Forster, 1956: Agrodiaetus altivagans sp. nov., Ordubad; Talysh, Zuvant, pag.Tatani

Tuzov, 1993: Agrodiaetus altivagans altivagans Forster, 1956, Armenian plateau

Tshikolovets, 2011: Polyommatus altivagans (Forster, 1956), S.W.Azerbaijan

Tschikolovets, Nekrutenko, 2012: Polyommatus altivagans altivagans (Forster, 1956), Nakhichevan, Zangezur Mts., Bitshenek

173. Polyommatus (Agrodiaetus) ectabanensis gorbunovi Dantchenko \& Lukhtanov, 1994

Dantchenko \& Lukhtanov, 1994: Polyommatus (Agrodiaetus) gorbunovi spec. nov., Talysh Mts., Zuvand, Mistan; Talysh, Zuvand, Golobin

Koçak, 1996: Polyommatus (Agrodiaetus) gorbunovi ssp. gorbunovi Dantchenko \& Lukhtanov, 1994, Talysh

Eckweiler \& Häuser, 1997: Polyommatus (Agrodiaetus) gorbunovi, Talysh, Zuvand

Dantchenko, 2000: Agrodiaetus gorbunovi (Dantchenko \& Lukhtanov, 1994), Zuvand Plateau, Talysh Mts.

Tuzov, 2000b: Agrodiaetus gorbunovi Dantchenko \& Lukhtanov, Talysh Mts., Zuvand Distr., Mistan

S.K.Korb, 2005: Polyommatus gorbunovi gorbunovi Dantchenko \& Lukhtanov, 1994, Talysh

Korb, Bolshakov, 2016: Polyommatus ectabanensis (de Lesse, 1963), Azerbaijan

174. Polyommatus (Agrodiaetus) turcicus turcicus (Koçak, 1977)

Tuzov, 1993: Agrodiaetus turcicus Kocak, 1977, Armenian plateau (Zangezursky Mts.)

Tshikolovets, 2011: Polyommatus turcicus (Koçak, 1977), Nakhichevan

Tschikolovets, Nekrutenko, 2012: Polyommatus turcicus turcicus (Koçak, 1977), Nakhichevan, Daralagez, Kyu-Kyu; Bitshenek pass.

175. Polyommatus (Agrodiaetus) firdussii firdussii (Forster, 1956)

Forster, 1960: Agrodiaetus actis pseudactis ssp. nov., Ordubad

Hesselbarth, van Oorschot \& Wagener, 1995: Polyommatus (Agrodiaetus) firdissii (Forster, 1956) (= pseudactis (Fortser, 1960 syn. nov.), Nachitschewan

Tuzov, 1993: Agrodiaetus firdussii pseudactis Forster, 1960, Armenian plateau (Daralagez); Talysh

Dantchenko, 2000: Pagrodiaetus firdussii Forster, 1956, Armenian Highland; Talysh

Dantchenko, 2000: Agrodiaetus firdussiissp. Firdussii Forster, 1956, Zuvand plateau, Talysh Mts.

Dantchenko, 2000: Pagrodiaetus firdussii ssp. pseudactis Forster, 1960, Armenian Highland; Talys Mts.

Tuzov, 2000e: Agrodiaetus firdussii pseudactis Forster, Nakhichevan, Buzgov; Talysh Mts., Zuvand Plateau

S.K.Korb, 2005: Agrodiaetus firdussii pseudactis Forster, 1960, Talysh

Tshikolovets, 2011: Polyommatus firdussii (Forster, 1956), Talysh, Gosmolyan; Nakhichevan

Tschikolovets, Nekrutenko, 2012: Polyommatus firdussii firdussii (Forster, 1956), Nakhichevan, Daralagez, Kyu-Kyu, Bitshenek

176. Polyommatus (Agrodiaetus) cyaneus cyaneus (Staudinger, 1899)

Staudinger, 1899: Lycaena Damone Ev. var. Cyanea, Hankynda

Forster, 1956: Agrodiatus Carmon cyanea Stgr., Hankynda; Talysh, Zuvand

Lukhtanov, 1989: Agrodiaetus cyaena (Staudinger, 1899), Nakhichevan, Ordubad distr, Nus-Nus

Tuzov, 1993: Polyommatus (Agrodiaetus) cyanea cyanea Staudinger, 1899, Armenian plateau; Talysh

Hesselbarth, van Oorschot \& Wagener, 1995: Polyommatus (Agrodiaetus) cyaneus cyaneus Staudinger, 1899, Nagorny Karabach

Koçak, 1996: Polyommatus (Agrodiaetus) cyanea ssp. cyanea Stgr., 1899, Nahcivan

Dantchenko, 2000b: Agrodiaetus cyaneus cyaneus (Staudinger, 1899), Talysh Mts. (Zuvand Plateau)

Tuzov, 2000b: Agrodiaetus cyaneus cyaneus Staudinger, Taslyh Mts., Zuvand Plateau

Tshikilovets, 2011: Polyommatus cyaneus cyaneus (Staudinger, 1899), Nagorno-Karabakh, Hankynda

Tschikolovets, Nekrutenko, 2012: Polyommatus cyaneus cyaneus (Staudinger, 1899), Talysh, Kosmolyan, Rosanov, Galabin, Hili-Dara; Nakhichevan, Zangezur Mts., Bitshenek; Daralagez, Kyu-Kyu

Korb, Bolshakov, 2016: Polyommatus cyaneus (Staudinger, 1899), Azerbaijan

177. Polyommatus (Agrodiaetus) cyaneus shamil(Dantchenko, 2000)

Dantchenko, 2000: Agrodiaetus shami/Dantchenko, spec. nov., Alty-Agach

Gorbunov, 2001: Plebejus shamil(Dantchenko, 2000), E.Azerbaijan 
S.K.Korb, 2005: Polyommatus shamil shamil Dantchenko, 2000, East Azerbaijan

\section{Family Riodinidae Grote, 1895}

178. Hamearis lucina lucina (Linnaeus, 1758)

Effendi, 1970: Nemeobius lucina L., Dashkesan, Bayan; Goygol Lake; Lachin, Yanshakh; Konakhkend, Erfi; Kusary, Dustair, Bedirkala

Tschikolovets, Nekrutenko, 2012: Hamearis /ucina lucina (Linnaeus, 1758), Lenkoran

Korb, Bolshakov, 2016: Hamearis lucina (Linnaeus, 1758), Azerbaijan

\section{Family Nimphalidae Swainson, 1827}

Subfamily Libytheinae Boisduval, 1833

179. Libythea celtis celtis (Laicharting, 1782)

Kolenati, 1846: Libythea Celtis, Fabr., Elisabethpol, Karabagh

Romanoff, 1884: Libythea Celtis Esp., Ordoubad

Effendi, 1970: Libythea celtis Laich., Dashkesan; Zakataly; Djari; Ordubad

Tuzov, 1993: Libythea celtis celtis Laicharting, 1782, Armenian plateau; Talysh

Tuzov, 2000b: Libythea celtis celtis (Laicharting, 1782), Nakhichevan, Pass Bichenek

Tschikolovets, Nekrutenko, 2012: Libythea celtis celtis (Laicharting, 1782), Jelisavetopol; Nkhichevan, Buzgov

Korb, Bolshakov, 2016: Libythea celtis (Laicharting, 1782), Azerbaijan

\section{Subfamily Danainae Boisduval, [1833]}

180. Danaus chrysippus chrysippus (Linnaeus, 1758)

Chrystoph, 1886: Danais Chrysippus L., Astara

Effendi, 1970: Danais chrysippus L., Talysh, Astara, Sarak, Artupa

Nekrutenko, 1990: Danais chrysippus (L.), Astara

Tuzov, 1993: Danaus chrysippus chrysippus (Linnaeus, 1758), Armenian plateau; Talysh

Murzin, 2000a: Danaus chrysippus chrysippus (Linnaeus, 1758), Nakhichevan; Lenkoran

Didmanidze, 2004: Danaus chrysippus (Linnaeus, 1758), Lenkoran distr., Astara

S.K.Korb, 2005: Danaus chrysippus Linnaeus, 1758, Astara

Korb, Bolshakov, 2016: Danaus chrysippus (Linnaeus, 1758), Azerbaijan

181. Melanargia russiae russiae (Esper, [1783])

Lederer, 1864: Melenargia clotho v. xenia H.-Sch., Helenendorf bei Elisabethpol

Wagner, 1975: Melanargia russiae caucasica (Nordmann, 1851), Helenendorf; Elisabethpol; Adji-Kent

Effendi, 1970: Melenargia japigia Cyr. ssp. caucasica Nordm., subalpine and alpine zones of the Greater and Lesser

Caucasus (ssp. sunarovius); the mountains of Nakhichevan (Paiz) and Talysh (Kelvyaz, Kyalakhan) (ssp. caucasica)

Didmanidze, 1979: Melanargia russiae Esp. sbsp. caucasica Nordm., Nakhichevan, Djulfa neighborhood

Tuzov, 1993: Melanargia russiae caucasica Nordmann, 1851, Armenian plateau; Talysh

Bogdanov, Samodurov \& Tuzov, 1997: Melanargia rusiae caucasica Nordmann, 1851 (= xenia Freyer, 1845), Armenian

Highland; Talysh

Tschikolovets, Nekrutenko, 2012: Melanargia russiae russiae (Esper, [1783]), Talysh, Zuvand

Korb, Bolshakov, 2016: Melanargia russiae (Esper, [1786]), Azerbaijan

Material: $10^{\pi}$, Nakhichevan, Shakhbuz, Bichenek, Shakhbuz National Park, 26.07.2016; 20º', Xyzi, 23.05.2014

\section{Melanargia larissa larissa (Geyer, [1828])}

Lederer, 1864: Melanargia hertha Hb. var. astanda Kdm., Helenendorf

Lederer, 1870: Melanargia Hertha Hb. et var. Astanda Kind., Helenedorf, Goektschai

Romanoff, 1884: Melanargia Larissa HS. var. Astanda Nordm., Ordoubad

Effendi, 1970: Melanargia larissa HB, Dashkesan, Khachbulag (Xaçbulağ); Kelbadjaru, Istisu; lachin, Agdavan; Kyapyaz Mt. Didmanidze, 1979: Melanargia larissa Hubn., Nakhichevan, Djulfa, geyser

Tuzov, 1993: Melanargia larissa astanda Staudinger, 1871, Armenian plateau

Tuzov, 1993: Melanargia larissa karabagi Kocak, 1976, Talysh

Bogdanov, Samodurov \& Tuzov, 1997: Melanargia larissa ssp. astanda (Boisduval, 1848), Nakhichevan, Zangezursky Mts., Bichenek Pass

Tschikolovets, Nekrutenko, 2012: Melanargia larissa larissa (Geyer, [1828]), Ordubad, Alakhi village; Bilav; Latshinsky distr., Kyurgadzhy village

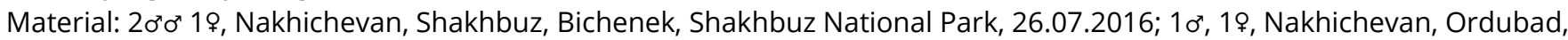

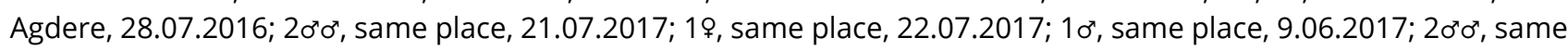
place, 9-12.06.2017; 19, Lerik, Gosmalyan, 23.06.2017 
183. Melanargia hylata hylata (Ménétriés, 1832)

Ménétriés, 1832: Satyrus hylata, mihi, Khanat de Talyche

Kolenati, 1846: Arge Hylata Ménétr., Talysch

Ménétriés, 1855: Arge Hylata, Mont. Talisch

Lederer, 1870: Melanargia Hylata Mén., Talyche

Romanoff, 1884: Melanargia Hylata Mén., Talyche

Christoph, 1886: Melanargia Hylata Mén., Talysch

Gaede, 1931: Melanargia hylata Ménétriés, Talysch

Effendi, 1970: Melanargia hylata Men., Talysh, Yardimli, Chayuzi; Lerik, Kosmolyan, Kyalyakhan, Pirasora Didmanidze, 1979: Melanargia hylata Men., Talysh

Nekrutenko, 1990: Melanargia hylata (Mén.), Talysh Mts., southern slopes of Zangezur Mts.

Tuzov, 1993: Melanargia hylata hylata Ménétriés, 1832, Armenian plateau; Talysh

Hesselbarth, van Oorschot \& Wagener, 1995: Melanargia hylata hylata (Ménétriés, 1832), Süden der Republik Aserbeidschan

Bogdanov, Samodurov, Tuzov, 1997: Melanargia hylata (Ménétriés, 1832), the southern slopes of the Zangezursky Mts., Tha Talysh Mts., Lerik Distr., Gosmalian

Bozano, 2002: Melanargia hylata hylata (Ménétriés, 1832), Zuvand, Talysh

S.K.Korb, 2005: Melanargia hylata hylata Ménétriés, 1832, Talysh; southern slope of Zangezursky Mts.

Tschikolovets, Nekrutenko, 2012: Melanargia hylata hylata (Ménétriés, 1832), Nakhichevan, Ms.Darydagh, pr. Dzhulfa; Shakhbuz, Kyu-Kyu; Talysh, Zuvand, Juchary Ambur-Dara, Dzhoni

Korb, Bolshakov, 2016: Melanargia hylata (Ménétriès, 1832), Azerbaijan

Material: 10", Nakhichevan, Ordubad, Agdere, 28-30.07.2016; 10", Lerik, Gosmalyan, 23.06.2017; 10", 19, Lerik, Gosmalyan, 21-22.06.2017

184. Melanargia teneates teneates (Ménétriés, 1832)

Ménétriés, 1832: Satyrus Teneates, mihi, Talyche, Zouvant

Kolenati, 1846: Arge Teneates, Ménétr., Talysch

Nordmann, 1851: Arge Titea Klug., Talysch

Ménétriés, 1855: Arge Teneates, Mont.Talisch

Ménétriés, 1859: Arge teneates, Menetr., montagnes de Talysche

Lederer, 1870: Melanargia Tithea var. Teneates Mén., Zouvent

Romanoff, 1884: Melanargia Titea Klug var. Teneates Mén., Talyche

Christoph, 1886: Melanargia Tithea var. Teneates Mén., Suant

Standfuss, 1892: Melanargia Teneates, Lenkoran

Heyne, [1895]: Melanargia Titea Klg. var. Teneates Mén., Lenkoran

Staudinger, 1901: Melenargia Titea Klug. v. Teneates Mén., Talysch

Effendi, 1970: Melenargia titea Klug. ssp. teneates Men., Talysh, Lerik

Nekrutenko, 1990: Melanargia teneates (Mén.), Talysh Mts.

Tuzov, 1993: Melanargia teneates Ménétriés, 1832, Talysh

Boddanov, Samodurov \& Tuzov, 1997: Melanargia teneates (Ménétriés, 1832), Talysh Mts., Lerik Distr., Gosmalian

Bozano, 2002: Melanargia teneates teneates (Ménétriés, 1832), S. E.Azerbaijan (Talysch)

S.K.Korb., 2005: Melanargia teneates teneates Ménétriés, 1832, Talish

Tschikolovets, Nekrutenko, 2012: Melanargia teneates teneates (Ménétriés, 1832), Ms Talysh, Dzhoni, Allar, Polikesh,

Yardymly-Avash

Korb, Bolshakov, 2016: Melanargia teneates (Ménétriès, 1832), Azerbaijan, Talysh

Material: 19, Lerik, Gosmalyan, 23.06.2017; 10', 19, same place, 21-22.06.2017

185. Melanargia galathea satnia Fruhstorfer, 1917

Romanoff, 1884: Melanargia Galathea L. var. Procida Hbst., Hankynda; Adjikent; Kedabeg; Istidara; Goktcha

Effendi, 1970: Melanargia galathea L., everywhere

Tschikolovets, Nekrutenko, 2012: Melanargia galathea satnia Fruhstorfer, 1917, Nakhichevan, Bitsheneksky forest;

Karabakh, Lisogory; Latshinsky distr., Kyurgadzha village; Dzhulfa vic., Dary-Dagh

Korb, Bolshakov, 2016: Melanargia galathea (Linnaeus, 1758), Azerbaijan

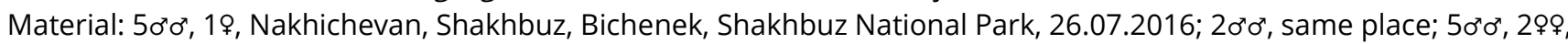

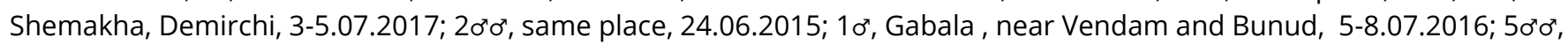

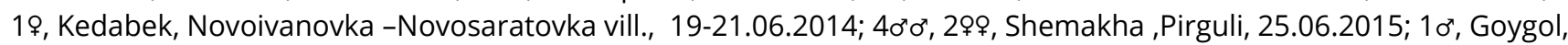

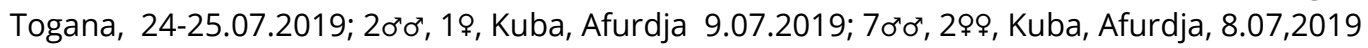

186. Protoerebia afra afra (Fabricius, 1787)

Fruhstorfer, 1918: Erebia afer zyxuta subspec. nova, Elisabethpol (Gjandzha)

Effendi, 1970: Erebia afer Esp., Nakhichevan, Paiz 
Tuzov, 1993: Proterebia afra hyrcana Staudinger, 1901, Armenian plateau; Talysh

Bogdanov, Samodurov \& Tuzov, 1997: Proterebia afra ssp.hyrcana (Staudinger, 1901), Armenian Highland; Talysh Tschikolovets, Nekrutenko, 2012: Protoerebia afra afra (Fabricius, 1787), Akstafa vic., Pojlu; Talysh, Zuvand, Kosmoljan; Elisavetpol (Krasnaja gora)

Korb, Bolshakov, 2016: Protoerebia afra (Fabricius, 1787), Azerbaijan

187. Erebia aethiops melusina Herrich-Schäffer, [1847]

Ménétriés, 1832: Satyrus Aethiops, Esp., Talyche

Lederer, 1864: Erebia Medea S.V., Helenendorf bei Elisabethpol

Romanoff, 1884: Erebia Aethiops Esp., Hankynda; Goktcha

Christoph, 1886: Erebia Aethiops Esp., Talysch

Jachontov, 1911: Erebia aethiops Esp. var. (subsp.) melusina H.-S., Ordubad; Ilkhi-Darasy (Elosavetpol); Goktsha

Effendi, 1970: Erebia aethiops Esp. ssp. melusina HS

Tschikolovets, Nekrutenko, 2012: Erebia aethiops melusina Herrich-Schäffer, [1847], Adzhi-kent

Korb, Bolshakov, 2016: Erebia aethiops (Esper, [1777]), Azerbaijan

188. Erebia melancholica melancholica Herrich-Shäffer, 1846

Effendi, 1970: Erebia melancholica HS., alpine zone of the Lesser Caucasus (Dali-Dag, Kapudjikh, Koshkar-Dag, Tfan, Gamysh Mts.)

Korb, Bolshakov, 2016: Erebia melancholica Herrich-Schäffer, [1846], Azerbaijan

189. Erebia medusa medusa ([Denis \& Schiffermüller], 1775]

Romanoff, 1884: Erebia Medusa F. var. Psoda Hb., Istissou

Effendi, 1970: Erebia medusa F., Dashkesen; Lachin, Yanshah, Agdavan; Kedabek; Istisu; Turshsu; Lake Goygol and

Maralgel

Tschikolovets, Nekrutenko, 2012: Erebia medusa medusa ([Denis \& Schiffermüller], 1775], Adzhi-kent, mts.Sarjal

Korb, Bolshakov, 2016: Erebia medusa (Fabricius, 1787), Azerbaijan

190. Erebia hewitsonii hewitsonii Lederer, 1864

Effendi, 1970: Erebia hewitsonii Ld., Babadag, Shakhdag, Bazardyuzi, Dalidag, Kyapaz, Mrovdag

S.K.Korb, 2005: Erebia hewitsonii hewitsonii Lederer, 1864, mountain ridges Murovdagh

Tschikolovets, Nekrutenko, 2012: Erebia hewitsonii hewitsonii Lederer, 1864, Adzhi-kent, mts. Sarjal

Korb, Bolshakov, 2016: Erebia hewitsonii Lederer, 1864, Azerbaijan

191. Erebia iranica dromulus Staudinger, 1901

Effendi, 1970: Erebia tyndarus Esp. ssp. dromulus Stgr., Lesser Caucasus, Turshsu

Bogdanov, Samodurov \& Tuzov, 1997: Erebia iranica Groum-Grshimailo, 1895, Nakhichevan, Zangezursky Mts., Bichenek Pass

Tshikolovets, 2011: Erebia iranica dromulus Staudinger, 1901, Nakhichevan, Alagez

Korb, Bolshakov, 2016: Erebia iranica Grum-Grshimailo, 1895, Azerbaijan

\section{Erebia graucasica transcaucasica Warren, 1950}

Lukhtanov, 1987: Erebia graucasica transcaucasica Warren, Mrovdag Mts., Khanlar; Karabakh plateau, Delidag Mt.

Lukhtanov, 1987: Erebia graucasica savalanica de Lesse, 1955, Ordubad

Nekrutenko, 1990: Erebia graucasica ssp., Dzhavakhet-Armenian plateau

Tuzov, 1993: Erebia graucasica ssp., Armenian plateau

Bogdanov, Samodurov \& Tuzov, 1997: Erebia graucasica? ssp. savalanica, de Lesse, 1955, Armenian Highland

Tshikolovets, 2011: Erebia graucasica transcaucasica Warren, 1950, S.-W.Azerbaijan

Korb, Bolshakov, 2016: Erebia graucasica Jachontov, 1909, Azerbaijan

193. Chortobius pamphilus pamphilus (Linnaeus, 1758)

Ménétriés, 1832: Satyrus Pamphilus, Linn, Lenkoran

Christoph, 1886: Coenonympha Pamphilus L., Lenkoran

Effendi, 1970: Coenonympha pamphilus L. ssp. Iyllus Esp., throughout the lowlands, in the foothills and mountains

Didmanidze, 1979: Coenonympha pamphilus L., Turianchay Reservation; Nakhichevan, Kyukyu, Paragachay river gorge,

Bilav; road to Ordubad; Shahbuz

Tuzov, 1993: Coenonympha pamphilus euxina Verity, 1926, Armenian plateau

Didmanidze, 2004: Coenonympha pamphilus Linnaeus, 1758, Turianchau Reservation; Nakhichevan, Kiu-Kiu; Paragachai river, Bilav, Ordubad; Mount Shakhbus 
Tschikolovets, Nekrutenko, 2012: Coenonympha pamphilus pamphilus (Linnaeus, 1758), Talysh, Lerik, Kosmoljan; Akstafa vic., Pojlu; Nakhichevan, Paragatshai; Zangezur Mts., Bitshenek village; Daralagez Mts., Buzgov Korb, Bolshakov, 2016: Chortobius pamphilus (Linnaeus, 1758), Azerbaijan

Material: $10^{\prime \prime}$, Nakhichevan, Ordubad, Agdere, 28-30.07.2016; 10", same place, 9.06.2017; 10", Along the Julfa-Ordubad

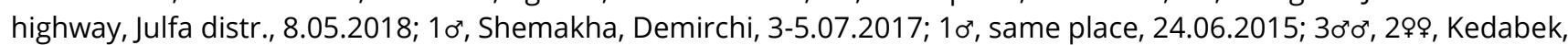

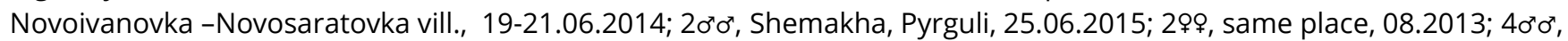
2\%9, Shemakha, Archiiman-Pirguli, near Shakhdag Milli Park,19-20.05.2013; 10', Zakatala, Djidjikhana, 10-12.07.2015; 10", 19, Belokani, Beshbulag, Djidjikhana, 25.06.2013

\section{Chortobius leander leander (Esper, [1784])}

Romanoff, 1884: Coenonympha Leander, Bakou

Effendi, 1970: Coenonympha leander Esp., everywhere

Tuzov, 1993: Coenonympha leander obscura Heyne, 1894, Armenian plateau; Talysh

Bogdanov, Samodurov \& Tuzov, 1997: Coenonympha leander obscura Heyne, [1894], Armenian Highland; Talysh;

Nakhichevan, Zangezursky Mts., Bichenek Pass; Kyukyu

Bozano, 2002: Coenonympha leander leander (Esper, 1784) (= obscura Heyne, 1894), Azerbaijan

Tschikolovets, Nekrutenko, 2012: Coenonympha leander leander (Esper, [1784]), Alty-Agatsh

Korb, Bolshakov, 2016: Chortobius leander (Esper, [1784]), Azerbaijan

Material: 10', Nakhichevan, Ordubad, Agdere, 9.06.2017

\section{Chortobius symphyta symphyta Lederer, 1870}

Sheljuzhko, 1929: Coenonympha symphyta symphyta, Adzhikent, Gouvern.Jelisavetpol

Effendi, 1970: Coenonympha symphyta Ld., Nakhichevan, Germachatakh; Gonagkend, Budug, Khinalig

Didmanidze, 1979: Coenonympha symphyta Ld., road to Baku, the neighborhood of the lake, "pass"

Didmanidze, 2004: Coenonympha symphyta Lederer, 1870, Baku district [misidentification]

Korb, Bolshakov, 2016: Chortobius symphita (Lederer, 1870), Azerbaijan

196. Chortobius glycerion glycerion (Borkhausen, 1788)

Ménétriés, 1832: Satyrus Iphis, Wien, montagnes de Talyche

Kolenati, 1846: Satyrus Iphis Hübn., Elisabethpol, Karabagh

Ménétriés, 1855: Coenonympha Iphis, Mont.Talish

Christoph, 1886: Coenonympha Iphis Schiff., Talysch

Effendi, 1970: Coenonympha iphis Schiff., everywhere in the forests of the foothills and mountains

Korb, Bolshakov, 2016: Chortobius glycerion (Borkhausen, 1788), Azerbaijan

\section{Chortobius arcania arcania (Linnaeus, 1761)}

Romanoff, 1884: Coenonympha Arcania L., Adjikent; Istissou

Effendi, 1970: Coenonympha arcania L. ssp. caucasica Jach., throughout the lowlands and in the foothills

Tuzov, 1993: Coenonympha arcania arcania Linnaeus, 1761, Armenian plateau

Bogdanov, Samodurov \& Tuzov, 1997: Coenonympha arcania arcania (Linnaeus, 1761) (= caucasica Jachontov, 1914),

Armenian Highland

Tschikolovets, Nekrutenko, 2012: Coenonympha arcania arcania (Linnaeus, 1761), Ordubad

Korb, Bolshakov, 2016: Chortobius arcania (Linnaeus, 1760), Azerbaijan

Material: $10^{\prime \prime}$, Nakhichevan, Shakhbuz, Bichenek, Shakhbuz National Park, 26.07.2016; 10, Shemakha, Pirguli, 28.06.2013

198. Chortobius saadi saadi (Kollar, 1848)

Ménétriés, 1832: Satyrus Dorus, Esp., montagnes de Talyche

Kolenati, 1846: Satyrus Dorus, Esp., In provincial Talysch

Nordmann, 1851: Coenonympha Dorus Esp., Gebirge in Talysh

Romanoff, 1884: Coenonympha Saadi Koll., Ordoubad; Adjikent

Christoph, 1886: Coenonympha Dorus, Esp., Talysch

Davenport, 1941: Coenonympha saadi saadi (= iphias Eversmann, 1851), Ordubad

Effendi, 1970: Coenonympha saadi Koll., Ordubad

Didmanidze, 1979: Coenonympha saadi Koll., Nakhichevan, the neighborhoods of Ordubad, Araks River gorge, the frontier; Julfa, geyser, Kvrak; Shahbuz; Paragachay river gorge, Bilav

D"Abrera, 1990: Coenonympha saadi Kollar, 1848, Ordubad

Tuzov, 1993: Coenonympha saadi saadi Kollar, 1850, Armenian plateau; Talysh

Bogdanov, Samodurov \& Tuzov, 1997: Coenonympha saadi saadi Kollar, Nakhichevan, Buzgov, Negram

Didmanidze, 2004: Coenonympha saadi Kollar, 1850, Ordubad, Araxi gorge; Julfa, Kivrak; Mount Shakhbus; Paragachai river, Bilav

Tshikolovets, 2011: Coenonympha saadi saadi (Kollar, [1849]), Ordubad 
Korb, Bolshakov, 2016: Chortobius saadi (Kollar, 1849), Azerbaijan

Material: 30"0", 19, Along the Julfa-Ordubad highway, Julfa distr., 8.05.2018; 30"0', 19, Nakhichevan, Shakhbuz distr., between the village of Mahmudoba and Nursu, 30.05.2018; 19, Nakhichevan, Sirab, 7.06.2017

199. Lasiommata petropolitana petropolitana (Fabricius, 1787)

Didmanidze, 1979: Lasiommata petropolitana Fabr., Nakhichevan, Ordubad

Didmanidze, 2004: Lasiommata petropolitana Fabricius, 1787, Nakhichevan, Ordubad

200. Lasiomata maera maera (Linnaeus, 1758)

Romanoff, 1884: Pararge Maera L. var. Adrasta Hb., Ordoubad

Sheljuzhko, 1937: Pararge maera abastumana (ssp. nov.), Elizavetpol (= Gandzha)

Effendi, 1970: Pararge maera L. ssp. monotonia Schilde, throughout the foothills of the Greater and Lesser Caucasus and Talysh (ssp. monatonia Schilde) and Nakhichevan - Araks valley, Paiz, Shahbuz, Norashen, Ordubad (ssp. orientalis Stgr.) Didmanidze, 1979: Lasiommmata maera L., Nakhichevan, Shakhbuz ridge

Tuzov, 1993: Lasiommmata maera orientalis Ruhl et Heyne, 1895, Armenian plateau; Talysh

Bogdanov, Samodurov \& Tuzov, 997: Lasiommata maera ssp. orientalis Rühl, [1894] (= armeniaca Sheljuzhko, 1937; = carsiana Sheljizhko, 1937), Armenian Highland; Talysh

Didmanidze, 2004: Lasiomata maera Linnaeus, 1758, Nakhichevan, Mount Shakh-Buz

Tschikolovets, Nekrutenko, 2012: Lasiomata maera maera (Linnaeus, 1758), Ordubad, Buzgov, Alakhi

Korb, Bolshakov, 2016: Lasiommata maera (Linnaeus, 1758), Azerbaijan

Material: $10^{\prime \prime}$, Nakhichevan, Ordubad, Agdere, 22.07.2017

201. Lasiommata adrastoides adrastoides (Bienert, 1870)

Christoph, 1874: Pararge Maera v. Adrastoides Bienert, Astrabad, Lenkoran

Romanoff, 1884: Pararge Maera v. Adrastoides Bien., Lenkoran, Talyche

Christoph, 1886: Pararge Maera, var. adrastoides Bienert, Lenkoran, Suvant

Heyne, [1894]: Pararge Maera L. v. adrastoides Bien., Astrahabad, Lenkoran

M. Korb., 1920: Pararge maera L. v. Adrastoides Bien., Lenkoran, Talysch-Gebirges

Effendi, 1970: Pararge adrastoides Bienert., forest belt along the Caspian coast from Lenkoran (Sutamurdov) to Astara (Sarak)

Korshunov, 1972: Lasiommata adrastoides Bienert, 1870, Talysh

Nekrutenko, 1990: Lasiommata adrastoides (Bienert), Talysh Mts.

Tuzov, 1993: Lasiommata adrastoides (Bienert, 1870), Talysh

Bogdanov, Samodurov \& Tuzov, 1997: Lasiommata adrastoides (Bienert, 1870), Talysh Mts., Zuvand; Lerik distr., Avrora S.K.Korb, 2005: Lasiommata adrastoides adrastoides Bienert, 1870, Talysh

Tschikolovets, Nekrutenko, 2012: Lasiommata adrastoides adrastoides (Bienert, 1870), Talysh, Bilesara, Polikesh env., Svakaran, Azerbaydzhan village, Azfilial

Korb, Bolshakov, 2016: Lasiommata adrastoides (Bienert, 1870), Azerbaijan

Material: 10, Lenkoran, Azfilial, 16-19.09.2013; 19, Massali, near Kubin and Mijankyu vill., 5.05.2014

202. Lasiommata megera megera (Linnaeus, 1767)

Effendi, 1970: Pararge megera L., throughout the lowlands and in the foothills

Didmanidze, 1979: Lasiommata megera L., Nakhichevan, Ordubad, Bilav; Paragachai river gorge

Tuzov, 1993: Lasiommata megera lyssa Boisduval, $1832 \mathrm{~m}$ Armenian plateau

Tuzov, 1993: Lasiommata megera iranica Riley, 1921, Talysh

Tschikolovets, Nekrutenko, 2012: Lasiommata megera megera (Linnaeus, 1767), Ordubad, Alakhi village; Kyurgadzhi;

Akstafa vic., Pojlu; Ms Talysh-Zuvand, Galabin; Elizavetpol

Korb, Bolshakov, 2016: Lasiommata megera (Linnaeus, 1767), Azerbaijan

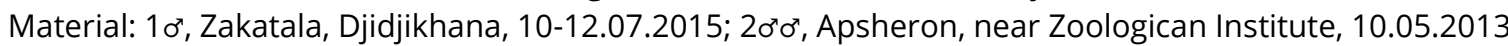

\section{Lasiommata menava menava Moore, 1865}

Romanoff, 1884: Pararge Menava Moore (= NasshreddiniChr.), Ordoubad

Nekrutenko, 1990: Lasiommata menava nasshreddini(Chr.), Ordubad vic., Nakhichevan

Tuzov, 1993: Lasiommata menava nasshreddini Christoph, 1877, Armenian plateau

Lukhtanov \& Lukhtanov, 1994: Lasiommata menava Moore, 1865, Ordubad

Tshikolovets, 2011: Lasiommata menava Moore, 1865 (= nasshreddini Christoph, 1877), S.Azerbaijan, Nakhichevan, Ordubad

204. Pararge aegeria tircis (Godart, 1821)

Kolenati, 1846: Satyrus Aegeria. Linn. var. Xiphia. Fabr., (in provincial Elisabethpol) 
Ménétriés, 1859: Lasiommata aegeria, Linn., Elisabethpol

Effendi, 1970: Pararge aegeria L., throughout the foothills and lowlands

Tuzov, 1993: Pararge aegeria tircis Godart, 1821, Armenian plateau; Talysh

Korb, Bolshakov, 2016: Pararge aegeria (Linnaeus, 1758), Azerbaijan

Material: $1 \sigma^{\prime \prime}$, Gabala, near Vendam and Bunud, 5-8.07.2016; 10, Shemakha, Pirguli, 08.2013; Ismailly Kaladjik, Velasin, 2123.07.2014; 10, Kuba, Afurdja, 8.07,2019

\section{Kirinia climene climene (Esper, [1783])}

Lederer, 1864: Pararge clymene Esp., Helenendorf bei Elisabethpol

Sheljuzhko, 1925: Pararge climene noxandra H.-S., Helenendorf, Jelizavetpol

Sheljuzhko, 1925: Pararge climene valentinae Miller, Karamalinovka (bei Nachitshevanj)

Effendi, 1970: Pararge climene Esp. ssp. caucasica Nordm., Shemakha, Kirovka (= climene Esp.); Khanlar (now Goygol) (= roxandra HS) and Nakhichevan, Ordubad (= valentinae Mill.)

Nekrutenko, 1990: Esperarge climene roxandra(H. - S.) (= valentinae Miller, 1923; = tkatshukoviSheljuzhko, 1925),

Dzhavakhet-Armenian plateau

Tuzov, 1993: Esperarge climene roxandra Herrich-Schaffer, 1850, Armenian plateau

Tshikolovets, 2011: Kirinia climene (Esper, [1783]), Alty-Agach

Tschikolovets, Nekrutenko, 2012: Kirinia climene climene (Esper, [1783]), Ordubad, Alakhi; Nakhichevan, Bitshenek; lac.Batabat; Alty-Agatsh; Shemakha, Kirovka

Korb, Bolshakov, 2016: Esperarge climene (Esper, [1783]), Azerbaijan

Material: 20`ơ, 19, Nakhichevan, Shakhbuz, Bichenek, Shakhbuz National Park, 26.07.2016

206. Apahntopus hyperanthus hyperanthus (Linnaeus, 1758)

Effendi, 1970: Apahntopus hyperatnus L., Khachmas, Kusarchay; Shemakha, Pirkuli; Nukha (now Sheki), Topchi; Zakatala, Djari; Lachin, Kamishli

207. Maniola jurtina phormia (Fruhstorfer, 1909)

Ménétriés, 1855: Satyrus Janira, Lenkoran

Becker, 1873: Epinephele Janira, Lenkoran

Romanoff, 1884: Epinephele Janira L. var. Hispulla Hb., Lenkoran

Christoph, 1886: Pararge Janira L., Lenkoran

Heyne, [1894], Epinephele Janira L., Lenkoran

Jachontov, 1911: Epinephele jurtina L., Elizavetpol (Khanagey, Karabag); Aresh; Lenkoran

Effendi, 1970: Epinephele jurtina L., throughout the Alpian belt

Tuzov, 1993: Maniola jurtina strandiana Oberthur, 1936, Armenian plateau

Bogdanov, Samodurov \& Tuzov, 1997: Maniola jurtina ssp. Strandiana Oberthür, 1936, Armenian Highland

Tschikolovets, Nekrutenko, 2012: Maniola jurtina phormia (Fruhstorfer, 1909), Ordubad, Bilav; Khurst village; Gosmolyan;

Bitshenek; Lerik; Latshinsky distr., Kurgadzha village; Nakhichevan, Batabat lac.; Adzhi-kent; Terter (gub. Elisavetpol)

Korb, Bolshakov, 2016: Maniola jurtina (Linnaeus, 1758), Azerbaijan

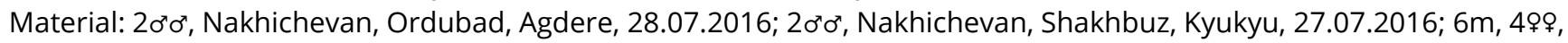
Astara, Toradi, 13-16.06.2016; 10", Shemakha, Demirchi, 3-5.07.2017; 10', same place, 24.06.2015; 10', Gabala , near

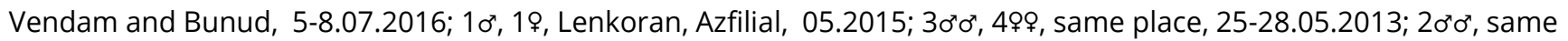
place, 26-28.05.2014; 20'o', 19, Kedabek, Novoivanovka -Novosaratovka vill., 19-21.06.2014; 19, Shemakha, Pirguli, 25.06.2015; 10', 3o\%, same place, jurtina ; $10^{\star}$, Zakatala, from kombinat to State Reserve, 24.06.2014; $10^{\prime \prime}$, Ismailly Kaladjik, Velasin, 21-23.07.2014; 10', Massali, near Kubin and Mijankyu vill., 5.05.2014

208. Hyponephele lupina intermedia (Staudinger, 1886)

Romanoff, 1884: Epinephele Lycaon Rott. var. Lupinus Costa, Ordoubad

Effendi, 1970: Epinephele lupinus Costa ssp. transcaucasica Jach., throughout the lowlands and in the foothills

Didmanidze, 1979: Hyponephele lupine Cost., Nakhichevan, Ordubad, Paragachay river gorge

Tuzov, 1993: Hyponephele lupine transcaucasica Jachontov, 1910, Armenian plateau

Samodurow, Tschikolovetz \& Korolew, 2001: Hyponephele lupina intermedia (Staudinger, 1886), Nakhichevan,

Bitschenek; W. Azerbajschan; Terter; Adzhi-Kent; Alty-Agatsh; Talysch, Suwand, Gosmoljan

Didmanidze, 2004: Hyponephele lupine Costa, 1836, Nakhichevan, Ordubad, Paragachai gorge

Tschikolovets, Nekrutenko, 2012: Hyponephele lupina intermedia(Staudinger, 1886), Ordubad, Bilav; Ms. Talysh-Zuvand, Rosanov

Korb, Bolshakov, 2016: Hyponephele lupina (Costa, [1836]), Azerbaijan

Material: $1 \sigma^{\prime \prime}$, Nakhichevan, Ordubad, Agdere, 28-30.07.2016; 10', 19, same place, 24.07.2017; 10', same place, 22.07.2017 ;

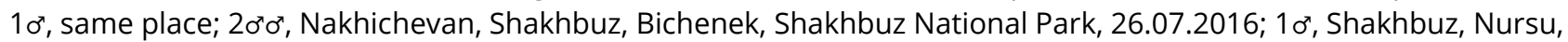

8.06.2017 
209. Hyponephele lycaon zuvandica Samodurov \& Korolev, 1996

Romanoff, 1884: Epinephele Lycaon Rott., Ordoubad; Zangesour

Effendi, 1970: Epinephele lycaon Rott., everywhere in the foothills and mountains

Didmanidze, 1979: Hyponephele lycaon Rott. sbsp. transcaucasica lach., Turianchai Reservation; nakhichevan,

Paragachai river gorge, Shakhbuz

Samodurov, Korolev \& Tshikolovets, 1996: Hyponephele lycaon zuvandica Samodurov \& Korolev, subsp. nov., Talysch, Zuvand, loc. Gosmoljan; Zuvand, loc.Gjuydara; Gilidara; Elisabetpol, Zangezur-Gebirgskette; Bitschenek; ZangezurskiGebergskette, loc. Njusnjus; Talysh, Zuvand, Ljaljakeran

Bogdanov, Samodurov \& Tuzov, 1997: Hyponephele lycaon ssp. zuvandica Samodurov et Korolev, 1996, Talysh Mts.; Nakhichevan region, Zangezursky Mts., Nyusnyus; Bichenek Pass

Samodurow, Tschikolovetz \& Korolew, 2001: Hyponephele lycaon zuvandica Samodurov et Korolev, 1996, Talysch, Suwand, Gosmoljan, Gjuidara, Gilidara; loc. bei Elisabetpol, Sangesurski-Gebk., Ordubad, Pasmara, Kjukju; DaralagesGebk., Bitschenek; Talysh, Siwand, Laljakeran; Bitshenek, loc.Batabat; Nakhichevan, Ms.Darydagh, pr. Dzhulfa; Nakhichevan, Nehram; Talysh-Suwand, Kosmoljan; Talysh, Hili-Dara; Ordubad, Alachi

Didmanidze, 2004: Hyponephele lycaon Rottemburg, 1774, Nakhichevan, Paragachai, Shakhbus

Eckweiler \& Bozano, 2011: Hyponephele lycaon ssp. zuvandica Samodurov \& Korolev, 1996 (= zuvandi Tuzov, 1993), Talysh, Zuvand, Gosmalian

Tshikolovets, 2011: Hyponephele lycaon zuvandica Samodurov \& Korolev, 1996, Nakhichevan, Nyusnyus, Bichenek Tschikolovets, Nekrutenko, 2012: Hyponephele lycaon zuvandica Samodurov \& Korolev, 1996, Ms.Talysh-Zuvand, Rosanov, Nal"vaz

Korb, Bolshakov, 2016: Hyponephele lycaon zuvandica Samodurov et Koroljev, 1996, Azerbaijan, Talysch, Zuvand, Ioc. Gosmoljan

Material: 10์, Nakhichevan, Shakhbuz, Bichenek, Shakhbuz National Park, 26.07.2016; 19, Along the Julfa-Ordubad highway, Julfa distr., 8.05.2018; 2000', 2\%ᄋ, Nakhichevan, Shakhbuz distr., between the village of Mahmudoba and Nursu, 30.05.2018; $1 \sigma^{x}$, Lerik, Gosmalyan, 23.06.2017

\section{Hyponephele lycanoides lycanoides D.Weiss, 1978}

Tuzov, 1993: Hyponophele lycanoides D.Weiss, 1978, Armenian plateau

Bogdanov, Samodurov \& Tuzov, 1997: Hyponephele lycanoides D.Weiss, 1978, Nakhichevan, Zangezursky Mts., Nyusnyus Samodurow, Tschikolovetz \& Korolew, 2001: Hyponephele lycanoides D.Weiss, 1978, Nachitschewan Reg., Sangesursky Gebk., Njusnjus

Eckweiler \& Bozano, 2011: Hyponephele lycanoides ssp. lycanoides D.Weiss, 1978, Azerbaijan

\section{Hyponephele naricina naricoides Gross, 1977}

Nekrutenko, 1990: Hyponephele naricina naricoides Gross, Talysh, Zuvand Tuzov, 1993: Hyponephele naricina Gross, 1977, Armenian plateau, Talysh

Bogdanov, Samodurov \& Tuzov, 1997: Hyponephele naricina Gross, 1977, Armenian Highland, Talysh, Lerik distr., Gosmalian; Talysh Mts. Dzhoni

Samodurow, Tschikolovetz \& Korolew, 2000: Hyponephele naricoides Gross, 1977, Dshulfa-Gegend; Talysch, Lerik-Rayon, Gosmoljan, loc. Kischlak; Nachitschewan, Buzgow, Daralages-Gebk.; Ordubad; Pasmara, Ordubad rayon; Nachitschewan, Daralages-Gebk., Nishni-Remeschin; Talysh, Suwand, Gjudara; Talysch, Suwand, Dshoni

S.K.Korb, 2005: Hyponephele naricoides naricoides Gross, 1977, Talysh; Nakhichevan

Eckweiler \& Bozano, 2011: Hyponephele naricina ssp. naricoides Gross, 1977, Azerbaijan

Tshikolovets, 2011: Hyponephele naricina naricoides Gross, 1977, Nakhichevan; Talysh

Tschikolovets, Nekrutenko, 2012: Hyponephele naricina naricoides Gross, 1977, Ms Talysh, Zuvans, pag. Rosanov

Korb, Bolshakov, 2016: Hyponephele naricina (Staudinger, 1870), Azerbaijan

\section{Hyponephele comara comara (Lederer, 1870)}

Romanoff, 1884: Epinephele Davendra Moore var. Comara Ld., Ordoubad Nekrutenko, 1990: Hyponephele comara Lederer, 1870, Armenian plateau

Bogdanov, Samodurov \& Tuzov, 1997: Hyponephele comara (Lederer, [1870]), Armenian Highland (the southern part of the Zangezursky Mts.), Nakhichevan Region, Zangezursky Mts., Nyusnyus; Zangezursky Mts., Ordubad

Samodurow, Korolew \& Tschikolowez, 1999: Hyponephele comara comara (Lederer, 1870), Ordubad, Niusnius

Didmanidze, 2004: Hyponephele comara Lederer, 1870, Nakhichevan, Ordubad

S.K.Korb, 2005: Hyponephele comara comara Lederer, 1870, Ordubad vicinity

Eckweiler \& Bozano, 2011: Hyponephele comara ssp. comara(Lederer, 1870), Nakhichevan

\section{Brintesia circe circe (Fabricius, 1775)}

Ménétriés, 1832: Satyrus Circe, Fab., Lenkoran

Kolenati, 1846: Satyrus Circe. Fabr. Proserpina. Hübn., Karabahg, Talysh

Ménétriés, 1855: Satyrus Proserpina, Lenkoran 
Lederer, 1864: Satyrus Proserpina S. V., Helenendorf bei Elisabethpol

Lederer, 1870: Satyrus proserpina S. V., Lenkoran

Christoph, 1877: Satyrus Circe F., Lenkoran

Romanoff, 1884: Satyrus Circe, Hankynda; Adjukent

Christoph, 1886: Satyrus Circe F., Lenkoran

Effendi, 1970: Satyrus circe F. ssp. venusta Frahst., throughout the foothills of the Greater and Lesser Caucasus and

Talysh

Didmanidze, 1979: Brintesia circe F., Turianchai Reservation

Didmanidze, 2004: Brintesia circe Fabricius, 1775, Turianchai Reservation

Tschikolovets, Nekrutenko, 2012: Brintesia circe circe (Fabricius, 1775), Adzhi-kent; Elisavetpol; Nakhichevan, Bitshenek Korb, Bolshakov, 2016: Brintesia circe (Fabricius, 1775), Azerbaijan

Material: 20"0', 19, Nakhichevan, Shakhbuz, Kyukyu, 27.07.2016; 10", Shemakha, Pirguli, 28.06.2013

214. Hipparchia parisatis parisatis (Kollar, [1849])

Romanoff, 1884: Satyrus Parisatis Koll., Ordoubad

Staudinger, 1901: Satyrus parisatis Koll. v. Macrophthalmus Ev., Ordubat

Jachontov, 1911: Satyrus parisatis Koll. var. (subsp.) macrophthalmus Ev., Aresh; Boz-Dagh Mt.

Le Cerf, 1913: Satyrus parisatis Koll. stultina n. ab., Ordoubad

Effendi, 1970: Satyrus parisatis Koll. macrophthalmus Ev.; Boz-Dag Hills system (in the area of Goychchay, Agdash and Mingachevour); Nakhichevan, Ordubad, Pais

Didmanidze, 1979: Neohipparchia parisatis Koll. (sbsp. macrophthalmus), Nakhichevan, Ordubad

Tuzov, 1993: Eumenis parisatis macrophtalma Eversmann, 1851, Armenian plateau

Bogdanov, Samodurov \& Tuzov, 1997: Hipparchia parisatis ssp. macrophthalma Eversmann, 1851, Armenian Highland Didmanidze, 2004: Hipparchia parisatis Kollar, 1850, Nakhichevan, Ordubad, Megrichai and Arpachai gorges

Tshikilovets, 2011: Hipparchia parisatis (Kollar, 1849), Ordubad

Tschikolovets, Nekrutenko, 2012: Hipparchia parisatis parisatis (Kollar, [1849]), Talysh; Nakhichevan, Nyusnyus;

Turantshay Nature Reserve

Korb, Bolshakov, 2016: Hipparchia parisatis (Kollar, 1849), Azerbaijan

Material: 1 m, Nakhichevan Sirab, 25.07.2016; 1 m, Nakhichevan, Badamli, 27.07.2016; 19, Nakhichevan, Shakhbuz,

Bichenek, Shakhbuz National Park, 26.07.2016

215. Hipparchia statilinus statilinus (Hufnagel, 1766)

Ménétriés, 1855: Satyrus Fauna, Lenkoran

Romanoff, 1884: Satyrus Statilinus Hufn., Elisabethpol

Effendi, 1970: Satyrus statilinus Hufn., Lerik, Gosmalyan; Fizuli, Goradiz (Horadiz); Ordubad

Tuzov, 1993: Eumenia statilinus Hufnagel, 1766, Armenian plateau

Tschikolovets, Nekrutenko, 2012: Hipparchia statilinus statilinus (Hufnagel, 1766), Nakhichevan, Daralagez, mount Buzgov; Gäncä (Kirovobad), Dashkhasan

Korb, Bolshakov, 2016: Hipparchia statilinus (Hufnagel, 1766), Azerbaijan

\section{Hipparchia fatua fatua Freyer, [1844]}

Nekrutenko, 1990: Hipparchia (Neohipparchia) fatua (Freyer), Nakhichevan

Tuzov, 1993: Eumenia fatua persiscana Verity, 1937, Armenian plateau

Bogdanov, Samodurov \& Tuzov, 1997: Eumenia fatua ssp. persiscana(Verity, 1937), Armenian Highland

Tschikolovets, Nekrutenko, 2012: Hipparchia fatua fatua Freyer, [1844], Nakhichevan, Zangezur, mont Bitshenek

\section{Hipparchia autonoe autonoe (Esper, [1783])}

Effendi, 1970: Satyrus autonoe Esp., Shemakha, Angekharan, Kirovka, Marazi-Kozluchay (Gozluçay)

Tschikolovets, Nekrutenko, 2012: Hipparchia autonoe autonoe (Esper, [1783]), Jelizavetpol

Korb, Bolshakov, 2016: Hipparchia autonoe (Esper, [1783]), Azerbaijan

218. Hipparchia syriaca syriaca (Staudinger, 1871)

Lederer, 1864: Satyrus Hermione L., Helenendorf bei Elisabethpol

Romanoff, 1884: Satyrus Hermione L., Lenkoran; Eldar

Effendi, 1970: Satyrus hermione L., everywhere in the foothills

Tuzov, 1993: Eumenis syriaca Staudinger, 1871, Armenian plateau

Bogdanov, Samodurov \& Tuzov, 1997: Hipparchia syriaca (Staudinger, 1871), Armenian Highland

Tschikolovets, Nekrutenko, 2012: Hipparchia syriaca syriaca (Staudinger, 1871), Lerik; Turiantshaysky Nature reserve;

Talysh, Orantshay gorge

Korb, Bolshakov, 2016: Hipparchia syriaca (Staudinger, 1871), Azerbaijan 
219. Hipparchia pellucida pellucida (Stauder, 1924)

Ménétriés, 1832: Satyrus Semele, Linn., montagnes de Talyche

Kolenati, 1846: Satyrus Semele. Linn., Elisabethpol, Karabagh

Ménétriés, 1855: Satyrus Semele, Lenkoran

Christoph, 1874: Satyrus Semele L., Lenkoran

Romanoff, 1884: Satyrus Semele L., Djebrail

Christoph, 1886: Satyrus Semele L., Talysh, Lenkoran

Effendi, 1970: Satyrus semele L., foothills of the Greater and Lesser Caucasus; Gobustan; Nakhichevan; Talysh; Bozdag hills

Kudrna, 1977: Hipparchia pellucida pellucida (Stauder) (= cotys Jachontov, 1935 syn. nov.), Azerbaydzhan

Didmanidze, 2004: Hipparchia pellucida Stauder, 1923, Nakhichevan, Julfa

Tschikolovets, Nekrutenko, 2012: Hipparchia pellucida pellucida(Stauder, 1924), Adzhi-kent; Elisavetpol (Krasnaja gora);

Terter

Korb, Bolshakov, 2016: Hipparchia pellucida (Stauder, 1923), Azerbaijan

220. Arethusana arethusa arethusa ([Dennis \& Schiffermüller], 1775)

Effendi, 1970: Satyrus arethusa Esp., Nagorny Karabakh (Dağlıg Garabağ), Shusha distr., Turshsu (Lisogorsk)

Tuzov, 1993: Arethusana arethusa pontica Heyne, 1895, Armenian plateau

Bogdanov, Samodurov \& Tuzov, 1997: Arethusana arethusa ssp. pontica (Heyne, [1895]), Armenian Highland

Tschikolovets, Nekrutenko, 2012: Arethusana arethusa arethusa ([Dennis \& Schiffermüller], 1775), Buzgov; Bitshenek;

Talysh-Zuvand, Gosmoljan; Nakhichevan, Zangezur, mont Bitshenek; Nakhichevan, Ordubad distr., Pazmara

Korb, Bolshakov, 2016: Arethusana arethusa ([Denis et Schiffermüller], 1775), Azerbaijan

Material: 20'0', 19, Nakhichevan, Ordubad, Agdere, 28-30.07.2016

221. Pseudochazara geyeri geyeri (Herrich-Shaäffer, [1846])

Effendi, 1970: Satyrus geyeri HS, Talysh, Kosmolyan; Nakhichevan, Ordubad, Paiz

Tuzov, 1993: Pseudochazara geyeri karsicola Gross, 1978, Armenian plateau

Bogdanov, Samodurov \& Tuzov, 1997: Pseudochazara geyeri ssp. karsicola Gross, 1978, Armenian Gighland

\section{Pseudochazara daghestana daghestana (Holik, 1955)}

Bogdanov, Samodurov \& Tuzov, 1997: Pseudochazara daghestana daghestana Holik, 1955, 100 km N Baku, Alty-Agach

Bogdanov, 2007: Pseudochazara daghestana daghestana (Holik, 1955), N. Azerbaijan, Shakhdagsky Mt.

Tshikolovets, 2011: Pseudochazara daghestana (Holik, 1955), Nakhichevan; Alty-Agach

Korb, Bolshakov, 2016: Pseudochazara daghestana (Holik, 1955), Azerbaijan

\section{Pseudochazara daghestana savalanica (Gross \& Ebert, 1975)}

Nekrutenko, 1989: Pseudochazara daghestana zangezura Nekrutenko, ssp. n., Nakhitchevan, Zangezur Mts., Bitshenek village

Nekrutenko, 1990: Pseudochazara daghestana zangezura Nekr., Zangezur Mts.

Tuzov, 1993: Pseudochazara daghestana savalanicus Gross et Ebert, 1975, Armenian plateau

Bogdanov, Samodurov \& Tuzov, 1997: Pseudochazara daghestana ssp. savalanica Gross et Ebert, 1975, Armenian Highland; Nakhichevan, Zangezursky Mts., Nyusnyus, Bichenek Pass

S.K.Korb, 2005: Pseudochazara daghestana zangezura Nekrutenko, 1989, Zangezur Mts.

Bogdanov, 2007: Pseudochazara daghestana savalanica (Gross et Ebert, 1975) (= zangezura Nekrutenko, 1989), Nyusnyus; Bitshenek pass., Ordubad

Tschikolovets, Nekrutenko, 2012: Pseudochazara daghestana savalanica (Gross \& Ebert, 1975), Nakhichevan, Ordubad distr., Pazmara village

Korb, Bolshakov, 2016: Pseudochazara daghestana zangezura Nekrutenko, 1989, Azerbaijan, Nakhichevan Republic, Zangezursky Mts., Bichenek

\section{Pseudochazara alpina alpina (Staudinger, 1878)}

Effendi, 1970: Satyrus alpina Stgr., Southern slopes of Mrovdag, Kyapyaz, Gyz-Yurdu Mts.

Bogdanov, Samodurov \& Tuzov, 1997: Pseudochazara alpina (Staudinger, 1878), Shakh-Dagh Mts., Laza

Material: $1 \sigma^{\prime \prime}$, Nakhichevan, Agdere, 24.07.2017; 1\%, Lerik, Gosmalyan, 23.06.2017

\section{Pseudochazara mamurra schahrudensis (Staudinger, 1881)}

Romanoff, 1884: Satyrus Pelopes Klug. var. Shahrudensis Stgr., Ordoubad

Tuzov, 1993: Pseudochazara schahrudensis schahrudensis, Staudinger, 1881, Armenian plateau; Talysh

Bogdanov, Samodurov \& Tuzov, 1997: Pseudochazara schahrudensis schahrudensis, (Staudinger, 1881), Armenian Highland; Talysh; Nakhichevan, Zangezursky Mts., Nyusnyus 
Didmanidze, 2004: Pseudochazara mamurra schahrudensis Staudinger, 1881, Nakhichevan, Julfa

Bogdanov, 2007: Pseudochazara schahrudensis schahrudensis, (Staudinger, 1881), Ordubad, Nyusnyus; Bitshenek pass.; Buzgov; Lerik, Gosmalyan, Dzhoni

Tshikolovets, 2011: Pseudochazara mamurra schahrudensis (Staudinger, 1881), S.Azerbaijan

Tschikolovets, Nekrutenko, 2012: Pseudochazara mamurra schahrudensis (Staudinger, 1881), Latshinsky distr., Kyurgadzhy village; Gosmolyan

Material: 2 m, Nakhichevan, Ordubad, Agdere, 28.07.2016; 10", same place, 21.07.2017; 10', 19, Lerik, Gosmalyan, 23.06.2017

\section{Pseudochazara pelopea persica (Christoph, 1877)}

Romanoff, 1884: Satyrus Pelopea Klug.var. Persica Stgr., Ordoubad

Heyne, [1895]: Satyrus Pelopea Klug var. caucasica Led., Helenendorf, Hankynda

Effendi, 1970: Satyrus pelopea Klug. ssp. caucasica Ld., Apsheron, Gobustan, Umbaki (Umbakı), Gyuzdek (Güzdek),

Angekharan, Marazi; Kedabek; Zangelan; Talysh, Kosmolyan; Nakhichevan, Shakhbuz, Ordubad; Bozdag Hills

Didmanidze, 1979: Pseudochazara pelopea Klug., Nakhichevan, Djulfa, Kivrak, Araks river gorge

Tuzov, 1993: Pseudochazara pelopea persica Staudinger, 1881, Talysh

Bogdanov, Samodurov \& Tuzov, 1997: Pseudochazara pelopea ssp. persica (Christoph, 1877), Armenian Highland; Talysh Didmanidze, 2004: Pseudochazara pelopea sbsp. persica Chr., Julfa, Kivrak

Bogdanov, 2007: Pseudochazara pelopea persica (Christoph, 1877), Bitshenek pass; Ordubad; Buzgov; Lerik; Zuvand, Gosmolyan, Dzhoni

Tschikolovets, Nekrutenko, 2012: Pseudochazara pelopea persica (Christoph, 1877), Talysh-Zuvand, Rosanov, Hili-Dara; Nakhichevan, Bilav

Korb, Bolshakov, 2016: Pseudochazara pelopea (Klug, [1832]), Azerbaijan

Material: $10^{x}$, Nakhichevan, Ordubad, Agdere 27.07.2018; $10^{x}$, same place, 21.07.2017; $10^{x}$, same place, 9.06.2017; $10^{*}$, 19, Nakhichevan, Shakhbuz distr., between the village of Mahmudoba and Nursu, 30.05.2018

\section{Pseudochazara beroe beroe (Freyer, 1843)}

Effendi, 1970: Satyrus beroe Frr., Norashen (now Sharur), Shakhbuz, Gernachatakh; Gosmolyan

Tuzov, 1993: Pseudochazara beroe rhena Herrich-Schaffer, 1852, Armenian plateau

Bogdanov, Samodurov \& Tuzov, 1997: Pseudochazara beroe ssp. rhena (Herrich-Schäffer, [1852]), Armenian Highland; Nakhichevan Region, Zangezursky Mts., Nyusnyus

Bogdanov, 2007: Pseudochazara beroe rhena (Herrich-Schaffer, 1852), Nakhichevan, Zangezur Mts., Bitshenek pass;

Zangezur Mts., near Ordubad, near Nyus-Nyus

Tshikolovets, 2011: Pseudochazara beroe (Freyer, 1843), W.Azerbaijan

Tschikolovets, Nekrutenko, 2012: Pseudochazara beroe beroe (Freyer, 1843), Nakhichevan, Ordubad distr.; Ganza Material: 1o', 1\%, Nakhichevan, Ordubad, Agdere, 11.06.2017

\section{Psedochazara thelephassa thelephassa (Geyer, [1827])}

Romanoff, 1884: Satyrus Telephassa Hb., Araxe; Nakhitchevan; Ordoubad

Effendi, 1970: Satyrus telephassa HB, Gobustan; Talysh, Kosmolyan; Nakhichevan, Norashen (Sharur), Ordubad; Bozdag Hills

Didmanidze, 1979: Psedochazara thelephassa Hb (?sbsp. persica Stgr.), Nakhichevan, Ordubad, Djulfa neighborhood, Nyusnyus

Nekrutenko, 1990: Psedochazara thelephassa (Geyer in Hb), Nakhichevan; Talysh (Zuvand)

Tuzov, 1993: Psedochazara thelephassa Geyer in Hubner, Armenian plateau; Talysh

Bogdanov, Samodurov \& Tuzov, 1997: Psedochazara thelephassa thelephassa (Geyer, [1827]), Armenian Highland;

Talysh; Nakhichevan region, Ordubad

Didmanidze, 2004: Psedochazara thelephassa Hübner, 1806, Julfa, Nius-Nius

S.K.Korb, 2005: Psedochazara thelephassa thelephassa Geyer in Hübner, [1827], Talysh

Bogdanov, 2007: Psedochazara thelephassa (Geyer, [1827]), Ordubad; Negram; Bitshenek pass; Buzgov; Gosmolyan

Tschikolovets, Nekrutenko, 2012: Psedochazara thelephassa thelephassa (Geyer, [1827]), Nahichevan, Bilav

Korb, Bolshakov, 2016: Pseudochazara thelephassa (Geyer, [1827]), Azerbaijan

Material: $10^{\prime \prime}$, Nakhichevan Sirab, 25.07.2016; 20"0', Along the Julfa-Ordubad highway, Julfa distr., 8.05.2018; $10^{\prime \prime}$,

Nakhichevan, Ordubad, Agdere, 28.07.2016; 10, 19, Nakhichevan, Shakhbuz near Makhmudoba and Nursu, 30.05 .2018

229. Chazara briseis meridionalis (Staudinger, 1886)

Romanoff, 1884: Satyrus Briseis L., Ordiubad

Jachontov, 1911: Satyrus briseis L. var. (subsp.) Fergana Stgr. et var. (subsp.) maracandica Stgr, Elisavetpol (Khanagey,

Karabag; Khamam-tshay, Ziarat Mt.)

Verity, 1937: Eumenis briseis L. race armena Jachontov, Jelizavetpol 
Effendi, 1970: Satyrus briseis L. ssp. armena Mill., throughout the foothills of the Greater Caucasus, less often in the Minor. In the foothills of the Talysh in abundance ssp. hyrcana Stgr.

Didmanidze, 1979: Chazara briseis L. sbsp. armena lach., Ordubad, Djulfa, geyser neighborhood

Tuzov, 1993: Chazara briseis major Oberthur, 1876, Armenian plateau

Tuzov, 1993: Chazara briseis hyrcana Staudinger, 1886, Talysh

Bogdanov, Samodurov \& Tuzov, 1997: Chazara briseis ssp. armena Jachontov, 1911, Armenian Highland; Talysh Mts., Lerik Distr., Gosmalian; Nakhichevan, Zangezursky Mts., Bichenek Pas

Didmanidze, 2004: Chazara briseis Linnaeus, 1764, Nakhichevan, Ordubad, Julfa

S.K.Korb, 2005: Chazara briseis armena Jachontov, 1911, Armenian plateau

Tschikolovets, Nekrutenko, 2012: Chazara briseis meridionalis (Staudinger, 1886), Diabar lowland; Alagez; Latshinsky distr., Kyurgodin; Bitshenek; Talysh, Kosmoljan; Elisavetpol; terter; Adzhi-kent

Korb, Bolshakov, 2016: Chazara briseis (Linnaeus, 1764), Azerbaijan

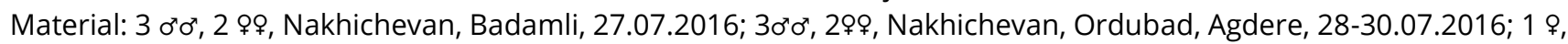

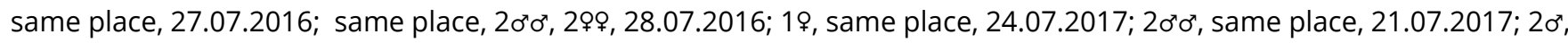
same place, 24.07.2017; 20"0", 19, 22.07.2017; 10", 19, Nakhichevan, Shakhbuz, Bichenek, Shakhbuz National Park,

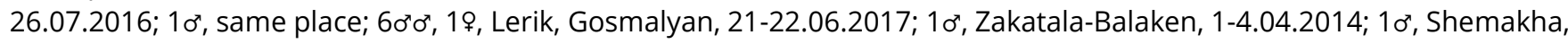
Demirchi, 24.06.2015

\section{Chazara persephone persephone (Hübner, [1805])}

Lederer, 1864: Satyrus anthe V. hanifa Nordm., Helenendorf

Romanoff, 1884: Satyrus Anthe O., Ordoubad; Zangesour; Alaghez

Romanoff, 1884: Satyrus Anthe O. var. Hanifa Nordm., Ordoubad

Effendi, 1970: Satyrus anthe O., Shemakha, Angekharan, Kirovka, Pirkuli; Gobakhkend, Kurkashen (now Gabala), Bum; Kakhi (Gakh), Ilisu; Nukha (Sheki): grain farm named after Ordzhonikidze

Didmanidze, 1979: Chazara anthe O., road to Gobustan, Tourogai Ridge; road to Baku, pass, lake surroundings Apsheron Peninsula; Nakhichevan: Djulfa, geyser, Araks river gorge, Kivrak, Paragachai river gorge

Tuzov, 1993: Chazara persephone transiens Zerny, 1932, Armenian plateau; Talysh

Bogdanov, Samodurov \& Tuzov, 1997: Chazara persephone ssp. transiens Zerny, 1932, Armenian Highland; Talysh; Nakhichevan Region, Daralagesky Mts., Remeshin

Didmanidze, 2004: Chazara anthe Ochsenheimer, 1807, Gobustan distr., Mount Turogai-Dag, Baku, Apsheron Range; Nakhichevan: Julfa, Araxi gorge, Kivrak, Paragachai gorge

Tschikolovets, Nekrutenko, 2012: Chazara persephone persephone (Hübner, [1805]), Ms Talysh, Kosmoljan;

Nakhichevan; Elisavetpol (Krasnaja gora)

Korb, Bolshakov, 2016: Chazara persephone (Hübner, [1804]), Azerbaijan

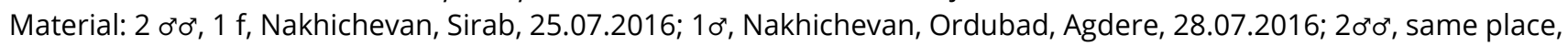
24.07.2017; 10", same place, 22.07.2017; 19, Lerik, Gosmalyan, 23.06.2017; 10, 19, Nakhichevan, Sirab, 7.06.2017

\section{Chazara bischoffii bischoffii (Herrich-Schäffer, [1846])}

Romanoff, 1884: Satyrus Bischoffii HS., Schoucha

De Freina \& Aussem, 1987: Chazara bischoffii, Nakhichevan

Effendi, 1970: Satyrus bischoffi HS, Ордубад, Паис

Didmanidze, 1979: Chazara bischoffii HS, Gobustan, ridgeTurogai; road to Baku, the pass of the lake; Nakhichevan, Julfa, Julfa-geyser, Araks gorge, Kivrak

Nekrutenko, 1990: Chazara bischoffii (H. - S.), Nakhichevan, Apheron peninsula, Kobystan, Talysh (Zuvand)

Tuzov, 1993: Chazara bischoffii bischoffii Herrich-Schaffer, 1851, Armenian plateau, Talysh

Bogdanov, Samodurov \& Tuzov, 1997: Chazara bischoffii (Herrich-Schäffer, [1846]), Talysh Mts., Lerik Distr., Zuvand Didmanidze, 2004: Chazara bischoffii Herrich-Schäffer, 1843-1845, Gobustan, Mount Turogai-Dag, Baku; Nakhichevan: Julfa, Araxi gorge, Kivrak

S.K.Korb, 2005: Chazara bischoffii bischoffii (Herrich-Schäffer, [1851]), Nakhichevan Region; Talysh: Zuvand; Apsheron Peninsula; Kobystan

Tshikolovets, 2011 Chazara bischoffii (Herrich-Schäffer, [1846]), S.and E.Azerbaijan (include Nakhichevan)

Tschikolovets, Nekrutenko, 2012: Chazara bischoffii bischoffii (Herrich-Schäffer, [1846]), Nakhichevan, Zangezur Mts. Bitshene; Talysh, Zuvand plateu, near Juhary Amburdara; Talysh, lerik distr., Gosmolyan; Talysh, Hili-Dara Korb, Bolshakov, 2016: Chazara bischoffi (Herrich-Schäffer, [1851]), Azerbaijan

Material: $10^{7}$, Nakhichevan, Ordubad, Agdere, 24.07.2017

\section{Minois dryas dryas (Scopoli, 1763)}

Ménétriés, 1832: Satyrus Phoedra, Linn., Lenkoran

Lederer, 1864: Satyrus Phaedra L., Elisabethpol

Romanoff, 1884: Satyrus Phaedra Dryas Scop., Hankynda; Goktcha

Sheljuzhko, 1937: Satyrus dryas Sc. ssp., Baku; Jelisavetpol; Adzhikent 
Effendi, 1970: Satyrus dryas L., everywhere in the forests of foothills and lowlands (Agdash, Garagan)

Tschikolovets, Nekrutenko, 2012: Satyrus dryas dryas (Scopoli, 1763), Adzhikent

Korb, Bolshakov, 2016: Minois dryas (Scopoli, 1763), Azerbaijan

233. Satyrus amasinus amasinus Staudinger, 1861

Romanoff, 1884: Satyrus Actaea Esp. var. Amasina Stgr., Ordoubad

Effendi, 1970: Satyrus actaea Esp. ssp. amasina Stgr., highlands in the area of Kazakh, Kedabek, Jebrail, Julfa and

Norashen (Sharur)

Korshunov \& Dubatolov, 1987: Satyrus amasinus Stgr., Talysh, Tshokara

Tuzov, 1993: Satyrus amasinis Staudinger, 1861, Armenian plateau

Tshikolovets, 2003: Satyrus amasinus, Alty-Agatsh

Bogdanov, 2008: Satyrus amasinus amasinus Staudinger, 1861, Ordubad, Buzgov

Tshikilovets, 2011: Satyrus amasinus amasinus S taudinger, 1861, Alty-Agatch

Korb, Bolshakov, 2016: Satyrus amasinus Staudinger, 1861, Azerbaijan

234. Satyrus iranicus iranicus Schwingenschuss, 1939

Korshunov \& Dubatolov, 1987: Satyrus iranica Schwing, Talysh, Tshokara

Nekrutenko, 1990: Satyrus iranicus Schwingenschuss, Talysh

Bogdanov, Samodurov \& Tuzov, 1997: Satyrus iranicus (Schwingenschuss, 1939), Talysh Mts., Lerik Distr., Zuvand,

Chokara

S.K.Korb, 2005: Satyrus iranicus iranicus Schwingenschuss, 1939, Talysh, Zuvand

Bogdanov, 2008: Satyrus iranicus iranicus Schwingenschuss, 1939, Talysh, Zuvand, Chokara; Gosmalyan

Tshikolovets, 2011: Satyrus iranicus (Schwingenschuss, 1939), Talysh

Tschikolovets, Nekrutenko, 2012: Satyrus iranicus iranicus Schwingenschuss, 1939, Talysh, Zuvand, Nal"vaz

Korb, Bolshakov, 2016: Satyrus iranicus Schwingenschuss, 1939, Azerbaijan

Material: 10", Nakhichevan, Ordubad, Agdere, 11.06.2017

235. Satyrus parthicus parthicus Lederer, 1869

Tshikolovets, 2011: Satyrus parthicus Lederer, 1869, W.Azerbaijan

Tschikolovets, Nekrutenko, 2012: Satyrus parthicus parthicus Lederer, 1869, Elisavetpol

236. Satyrus effendi effendi Nekrutenko, 1989

Nekrutenko, 1989: Satyrus effendi Nekrutenko, 1989: Nakhichevan, Ordubad distr., Pazmara village; Zangezursky Mts., Nyus-Nyus

Nekrutenko, 1990: Satyrus effendi Nekrutenko, 1989, South part of Zangezur Mts.

Tuzov, 1993: Satyrus effendi Nekrutenko, 1989, Armenian plateau

Bogdanov, Samodurov \& Tuzov, 1997: Satyrus effendi Nekrutenko, 1989, Nakhichevan Region, Zangezursky Mts., Nyusnyus

S.K.Korb, 2005: Satyrus effendi effendi Nekrutenko, 1989, Zangezur Mts.

Bogdanov, 2008: Satyrus effendi Nekrutenko, 1989, Paragachai, Kapudzhikh, Pazmara, Nyusnyus

Korb, Bolshakov, 2016: Satyrus effendi Nekrutenko, 1989, Azerbaijan, Nakhichevan Republic, Pazmara in Ordubad distr.

Subfamily Apaturinae Boisduval, 1840

237. Apatura ilia ilia ([Dennis \& Schiffermüller], 1775)

Effendi, 1970: Apatura ilia Schiff. ab clytie Schiff.(?) var. eos hossi, Belokany, Ah-Kimal, the territory Zakataly Reserve

238. Thaleropsis ionia ionia (Fischer de Waldheim \& Eversmann, 1851)

Romanoff, 1884: Thaleropsis ionia Ev., Ordoubad

Bramson, 1890: Thaleropsis ionia Ev., Ordubad

Rühl, [1893]: Thaleropsis Jonia Eversm., Ordubad

Effendi, 1970: Thaleropis jonia Ev., Nakhichevan, Ordubad, Pazmara

Tuzov, 1993: Thaleropsis jonia jonia Eversmann, 1851, Armenian plateau

Hesselbarth, van Oorschot \& Wagener, 1995: Thaleropsis ionia (Eversmann, 1851), Aserbeidschan

Tuzov, 2000b: Thaleropsis ionia Eversmann, 1851, Nakhichevan

Didmanidze, 2004: Thaleropsis jonia Fischer de Waldheim \& Eversmann, 1851, Nakhichevan, Nius-Nius

Masui, Bozano \& Floriani, 2011: Thaleropsis ionia (Eversmann, 1851), Nakhichevan

Tshikolovets, 2011: Thaleropsis ionia(Eversmann, 1851), Nakhichevan

Tschikolovets, Nekrutenko, 2012: Thaleropsis ionia ionia (Fischer de Waldheim \& Eversmann, 1851), Nakhichevan,

Ordubad distr., Pazmara

239. Limenitis reducta reducta Staudinger, 1901 
Ménétriés, 1832: Nymphalis Camilla, Fab., montagnes de Talyche

Kolenati, 1846: Limenitis Camlla Fabr., Elizabethpol, Talysch

Nordmann, 1851: Limenitus Camilla F. H., Talysh

Lederer, 1870: Limenitis Camilla S. V. Talyche, Hankynda

Christoph, 1886: Limenitis Camilla Schiff., Talysch

Effendi, 1970: Limenitis reducta ssp. reducta Staudinger, 1901, anywhere in the foothills and mountains up to 2000 m.

a.s.I.

Didmanidze, 1979: Limenitis reducta St., Turianchai Reservation

Tuzov, 1993: Limenitis reducta mirzajaniGross et Ebert, 1975, Armenian plateau; Talysh

Murzin, 2000b: Limenitis reducta reducta Staidonger, 1901, Armenian plateu; Talysh

Tuzov, 2000b: Limenitis reducta reducta Staudinger, 1901, Nakhichevan, Buzgov near Ordubad

Didmanidze, 2004: Limenitis reducta Staudinger, 1901, Turianchai Reservation

Tschikolovets, Nekrutenko, 2012: Limenitis reducta reducta Staudinger, 1901, Latshinsky dostr., Kurdtshadin; 10 km N Lerik; Shemakha

Korb, Bolshakov, 2016: Limenitis minor (Hufnagel, 1766) (= reducta Staudinger, 1901), Azerbaijan, Hankynda

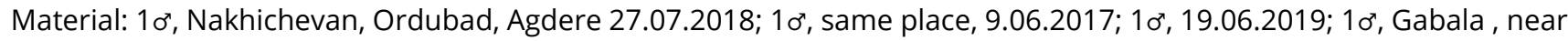

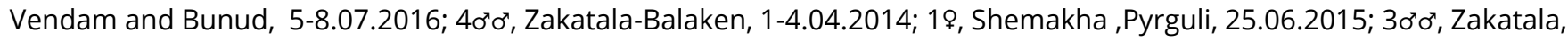

Djidjikhana, 10-12.07.2015; 20"0", Ismailly Kaladjik, Velasin, 21-23.07.2014

\section{Limenitis camilla camilla (Linnaeus, 1764)}

Effendi, 1970: Limenitis minitis ssp. camilla Schiff., anywhere in the foothills and mountains until 2000 m. a.s.l.

Didmanidze, 1979: Limenitis Camilla L., Apsheron peninsula, Khachmaz

Tuzov, 1993: Limenitis camilla camilla Linnaeus, 1764, Armenian plateau

Murzin, 2000b: Limenitis camilla (Linnaeus, 1764), Armenian Highland

Tschikolovets, Nekrutenko, 2012: Limenitis camilla camilla (Linnaeus, 1764), Adzhi-kent; Zurnabad (prope Elosavetpol)

Korb, Bolshakov, 2016: Limenitis Camilla (Linnaeus, 1764), Azerbaijan

\section{Neptis rivularis rivularis (Scopoli, 1763)}

Romanoff, 1884: Limenitis Lucilla F. ver. Ludmilla HS, Ordoubad

Effendi, 1970: Neptis lucilla F. ssp. Iudmilla HS., Dashkesan, Aterk, Agdavan, Yanshakh, Zorkeshish

Didmanidze, 1979: Neptis rivularis Scop., Nakhichevan, Ordubad

Tuzov, 1993: Neptis rivularis ludmilla Herrich-Shaffer, 1856, Armenian plateau; Talysh

Didmanidze, 2004: Neptis rivularis Scopoli, 1763, Nakhichevan, Ordubad, Julfa

Tschikolovets, Nekrutenko, 2012: Neptis rivularis rivularis (Scopoli, 1763), Elisavetpol

Korb, Bolshakov, 2016: Neptis rivularis (Scopoli, 1763), Azerbaijan

Material: $1 \sigma^{\prime}$, Nakhichevan, Shakhbuz, Bichenek, Shakhbuz National Park, 26.07.2016

242. Polygonia c-album c-album (Linnaeus, 1758)

Becker, 1873: Vanessa C-album, Lenkoran

Christoph, 1874: Vanessa C-album L., Lankoran

Christoph, 1886: Vanessa C-album L., Lankoran

Effendi, 1970: Polygonia C-album L., everywhere on the lowlands, in the foothills and mountains (up to $2200 \mathrm{~m}$ )

Didmanidze, 1979: Polygonia C-album L., Turianchai Reservation

Tschikolovets, Nekrutenko, 2012: Polygonia c-album c-album (Linnaeus, 1758), Latshinsky distr., Kyurdtshadzhi; Adzhi-

kent; Elisavetpol

Korb, Bolshakov, 2016: Polygonia c-album (Linnaeus, 1758), Azerbaijan

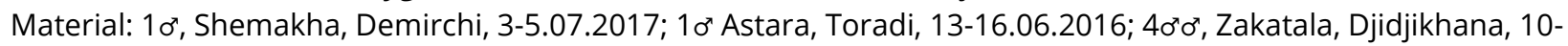

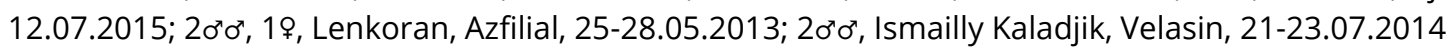

243. Polygonia egea egea (Cramer, [1775])

Romanoff, 1884: Vanessa Egea Cr., Ordoubad

Effendi, 1970: Polygonia egea Cr., Talysh, Zuvand, Kyalvyaz, Kyalyakhan; Nakhichevan, Ordubad

Didmanidze, 1979: Polygonia egea Cr., Nakhichevan, Nyusnyus, Kivrak

Tuzov, 1993: Polygonia vau-album Esper, 1780 (= egea Cramer, 1775), Armenian plateau; Talysh

Didmanidze, 2004: Polygonia egea egea Cramer, 1775, Nakhichevan, Nius-Nius, Kivrak

Tschikolovets, Nekrutenko, 2012: Polygonia egea egea (Cramer, [1775]), Nakhichevan, Ordubad distr., Pazamara

Korb, Bolshakov, 2016: Polygonia egea(Cramer, [1775]), Azerbaijan 
Korb, Bolshakov, 2016: Nymphalis xanthomelas (Esper, [1781]), Azerbaijan

245. Nymphalis polychloros polychloros (Linnaeus, 1758)

Ménétriés, 1832: Vanessa Polychloros, Linn., Lenkoran

Christoph, 1877: Vanessa polychloros L., Lenkoran, Asterabad

Christoph, 1886: Vanessa polychloros Bgstr., Lenkoran

Effendi, 1970: Vanessa polychloros L., everywhere in the foothills

Tuzov, 1993: Nymphalis polychloros fervida Standfuss, 1896, Armenian plateau; Talysh

Korb, Bolshakov, 2016: Nymphalis polychloros (Linnaeus, 1758), Azerbaijan

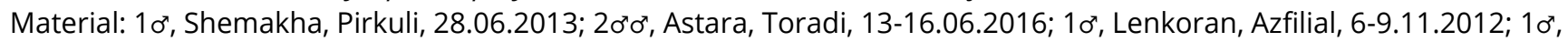
2\%o, same place, 26-28.05.2014

246. Nymphalis antiopa antiopa (Linnaeus, 1758)

Ménétriés, 1832: Vanessa Antiopa, Linn., Lenkoran

Kolenati, 1846: Vanessa Antiopa Linn., Talysch

Nordmann, 1851: Vanessa Antiopa L., Lenkoran

Christoph, 1886: Vanessa Antiopa L., Lenkoran

Effendi, 1970: Vanessa antiopa L., Belokany, Akh-Kimal

Korb, Bolshakov, 2016: Nymphalis antiopa (Linnaeus, 1758), Azerbaijan

247. Vanessa atalanta atalanta (Linnaeus, 1758)

Becker, 1873: Vanessa Atalanta L., Lenkoran

Christoph, 1877: Vanessa Atalanta L., Lenkoran

Christoph, 1886: Vanessa Atalanta L., Lenkoran

Stichel, 1911: Pyrameis atalanta L., Lenkoran

Effendi, 1970: Pyrameis atalanta L., everywhere from lowland to subalpine zone

Tschikolovets, Nekrutenko, 2012: Vanessa atalanta atalanta (Linnaeus, 1758), Elisavetpol; Adzhi-kent (prope Elisavetpol); Zakataly vic., Memabash

Korb, Bolshakov, 2016: Vanessa atalanta (Linnaeus, 1758), Azerbaijan

Material: 10 ', Nakhichevan, Shakhbuz, Bichenek, Shakhbuz National Park, 26.07.2016; 10', Shemakha, Pirkuli, 28.06.2013; 10", Astara,Toradi, 13-16.06.2016; 10", Siyazan, Beshbarmak, 4.06.2015; 10", Goygol, Togana, 24-25.07.2019

248. Vanessa cardui cardui(Linnaeus, 1758)

Ménétriés, 1832: Vanessa cardui, Linn., Lenkoran

Becker, 1873: Vanessa Cardui, Lenkoran

Christoph, 1886: Vanessa cardui L., Lenkoran

Effendi, 1970: Pyrameis cardui L., everywhere, from the coast to the subalpine zone

Didmanidze, 1979: Vanessa cardui L., Nakhichevan, Djulfa, geyser, Paragachai river gorge, Kyukyu; road to

Kyzylagadzhsky reserve; Mugan steppe, Salyan neighborhood

Tuzov, 1993: Vanessa cardui cardui Linnaeus, 1758, Armenian plateu; Talysh

Tshikolovets, 2003: Vanessa cardui, Alty-Agatsh

Tschikolovets, Nekrutenko, 2012: Vanessa cardui cardui (Linnaeus, 1758), Adzhi-kent

Korb, Bolshakov, 2016: Vanessa cardui (Linnaeus, 1758), Azerbaijan

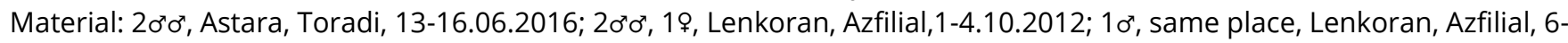
9.11.2012; 10', Shemakha, Pirguli, 28.06.2013; 19, Zakatala, from kombinat to State Reserve, 24.06.2014; 10', Apsheron, near Zoologican Institute, 10.05.2013; 30 $0^{\star}, 1$, Kuba, Afurdja 9.07.2019

249. Aglais urticae urticae (Linnaeus, 1758)

Christoph, 1886: Vanessa urticae L., Lenkoran

Effedi, 1970: Vanessa urticae L., everywhere in the foothills and mountains

Didmanidze, 1979: Aglais urticae L., Pirkuli reservation, Dyubrar ridge

Tuzov, 1993: Aglais urticae turcica Staudinger, 1871, Armenian plateau; Talysh

Korb, Bolshakov, 2016: Aglais urticae (Linnaeus, 1758), Azerbaijan

Material: 10', Lerik, Gosmalyan, 21-22.06.2017; 10, 2\%क, Shemakha, Archiman-Pirguli, near Shakhdag Milli Park,19-

20.05.2013

250. Inachis io io (Linnaeus, 1758)

Ménétriés, 1832: Vanessa io Linn., Lenkoran

Becker, 1873: Vanessa lo, Lenkoran

Christoph, 1877: Vanessa lo L., Lenkoran 
Christoph, 1886: Vanessa lo L., Lenkoran Jachontov, 1911: Vanessa io m. caucasica, Lenkoran

Gaede, 1930: Vanessa io nigromaculata Kleinschm., Elisabethpol

Effendi, 1970: Vanessa io L., everywhere in the foothills

Tschikolovets, Nekrutenko, 2012: Inachis io io (Linnaeus, 1758), Elizavetpol

Korb, Bolshakov, 2016: Inachis io (Linnaeus, 1758), Azerbaijan

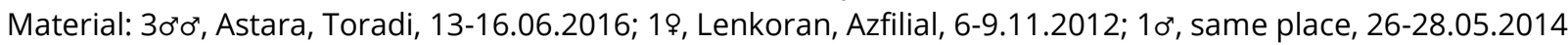

251. Araschnia levana levana (Linnaeus, 1758)

Ménétriés, 1832: Vanessa Prorsa, Linn., Lenkoran

Kolenati, 1846: Vanessa Prorsa, Linn. Levana L., (var.vernalis), Talysch

Nordmann, 1851: Vanessa Prorsa L., Lenkoran

Lederer, 1870: Vanessa Prorsa L. et var. Levana L., Talyche

Christoph, 1886: Vanessa Prorsa L. et var. Levana L., Lenkoran

Effendi, 1970: Arachnia levana L., Lachin, Yanshakh; Adjikent; Dashkesan

Korb, Bolshakov, 2016: Araschnia levana (Linnaeus, 1758), Azerbaijan

252. Euphydryas maturna staudingeriWnukowsky, 1929

Subfamily Melitaeinae Newman, 1869

Effendi, 1970: Melitaea maturna L. hossi, Lake Goygol, Maralgel, the southern slopes of Mrovdag (Yanshah, Agdavan); Kapudjik Mt.

253. Euphydryas aurinia bulgarica (Fruhstorfer, 1917)

Effendi, 1970: Melitaea aurinia Rott., Kuba, Dustair, Kutkashen (Gabala), Laza; Shemakha, Kirovka (ssp. aurinia Rott.);

Kakhi, Ilisu, Saribash (Sarıbaş) (ssp. pellucida Chr.); Nakhichevan, Norashen (Sharur) (ssp. orientalis HS)

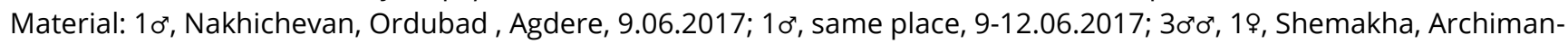
Pirguli, near Shakhdag Milli Park,19-20.05.2013

\section{Melitaea caucasogenita caucasogenita Verity, 1930}

Jachontov, 1911: Melitaea athalia Rott. ver. (subsp.) caucasica Stgr., Elisavetpol; Tshaj-Kend

Effendi, 1970: Melitaea athalia Rott. ssp. caucasica Stgr., in the mountains of the Greater and Lesser Caucasus, Talysh and Nakhichevan

Devyatkin, 2000: Melitaea caucasogenita caucasogenita Verity, 1930 (= caucasica Staudinger, 1871), Armenian Highland

Tshikolovets, 2003: Melitaea caucasogenita, Alty-Agatsh

Tshikolovets, 2011: Melitaea caucasogenita Verity, 1930, Alty-Agach

Korb, Bolshakov, 2016: Melitaea caucasogenita Verity, 1930, Azerbaijan

255. Melitaea aurelia ciscaucasica Rjabov, 1926

Effendi, 1970: Melitaea aurelia Wick. ssp. rhaelica Frey., Kelbadjar, Istisu; Lachin, Kamishli; Shusha, Turshsu Material: 19, Kedabek, Novosaratovka, Novoivanovka, 21.06.2014

\section{Melitaea diamina diamina (Lang, 1789)}

Romanoff, 1884: Meliteae Dictynna Esp., Kedabeg; Adjikent

Effendi, 1970: Meliteae dyctynna Esp., Shemakha, Pirkuli; Lachin, Yanshakh

Tschikolovets, Nekrutenko, 2012: Melitaea diamina diamina (Lang, 1789), Elisavetpol

Korb, Bolshakov, 2016: Melitaea diamina (Lang, 1789), Azerbaijan

257. Melitaea interrupta interrupta Kolenati, 1846

Ménétriés, 1832: Argynnia Didyma, Talych

Romanoff, 1884: Melitaea Dalmatina Stgr., Ordoubad

Christoph, 1886: Melitaea didyma O., Talysch

Staudinger, 1901: Melitaea Didyma O. v. Caucasica Stgr., Helenendorf, Hankynda

Effendi, 1970: Melitaea dydyma Esp. ssp. caucasica Stgr. (= ssp. alpina Stgr.; ssp. meridionalis Stgr.; = ssp. persea Koll.; = ssp. neera F.), throughout the lowlands, in the foothills and mountains

Lukhtanov \& Kuznetsova, 1989: Melitaea transcaucasica Turati, Nakhichevan, Zangezursky Mts., Ordubad

Tuzov, 1993: Melitaea didyma transcaucasica Turati, 1919, Armenian plateau, Talysh

Tshikolovets, 2003: Melitaea interrupta, Alty-Agatsh

Tshikolovets, 2011: Melitaea interrupta Kolenati, 1846 (= transcaucasica Turati, [1920]); = caucasi Verity, 1922; = venosata Niesiolowski, 1937; = musatscherii Alberti, 1969; = itkolensis Alberti, 1969), Alty-Agach 
Tschikolovets, Nekrutenko, 2012: Melitaea interrupta interrupta Kolenati, 1846, Talysh, Lerik distr., Gosmolyan; TalyshZuvand, Divagatsh, Gosmolyan, Goveri; Nakhichevan, Daralagez Mts., Kyukyu vill., Zangezur Mts., Bitshenek vill.; Kirovobad (Ganja)

Korb, Bolshakov, 2016: Melitaea interrupta Kolenati, 1846, Azerbaijan

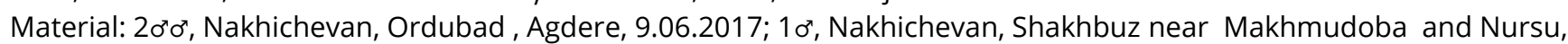
30.05.2018; $20^{\circ} 0^{7}$, Nakhiichevan, Shakhbuz, Nursu, 15.06.2019; 10, 1\%, Shemakha, Pyrguli, 25.06.2015; 10, 19, Kuba, Afurdja, 9.07.2019

258. Melitaea persea caucasica Staudinger, 1861

Romanoff, 1884: Melitaea Dalmatina Stgr. var. Persea Koll., Ordoubad; Hankynda

Chrystoph, 1889: Melitaea Didyma O. var. KaschtschenkoiChr., Jelisawetpol

Higgins, 1941: Melitaea persea caucasica Stgr., Elizabethpol

D"Abrera, 1992: Melitaea persea caucasica Herrich-Schaffer, Helenendorf

Tuzov, 1993: Melitaea persea arartica Verity, 1929, Armenian plateau, Talysh

Tshikolovets, 2003: Melitaea persea, Dzhulfa; Jenikend; Talysh, Hili-Dara

Tshikolovets, 2011: Melitaea persea caucasica Staudinger, 1861 (= kaschtschenkoi Christoph, 1889; = araratica Verity, 1929; = caucasicola Bryk, 1940), Julfa; Jenikend; Talysh, Hili-Dara

Tschikolovets, Nekrutenko, 2012: Melitaea persea caucasica Staudinger, 1861, Nakhichevan, Daralagez Mts., Kyu-Kyu; Talysh, Zuvand; Talysh, Lerik distr., Gosmolyan, Goveri, Hili-Dara, Divagatsh; Ordubad; Dzhulfa vic., Dary-Dagh; Turintshay; Akstafa vic., Pojlu; Nakhichevan, Paraga-tshay

Korb, Bolshakov, 2016: Melitaea persea Kollar, [1849], Azerbaijan

Mtaerial: $1 \sigma^{\prime \prime}$, Nakhichevan, Ordubad, Agdere, 9.06.2017; $10^{\prime \prime}$, Shemakha, Demirchi, 3-5.07.2017

259. Melitaea phoebe ottonis Fruhstorfer, 1917

Romanoff, 1884: Melitaea Phoebe Knoch, Talyche; Ordoubad

Christoph, 1886: Melitaea Phoebe Knoch, Talysch

Effendi, 1970: Melitaea phoebe Knoch. ssp. caucasica Stgr., in the foothills of the Greater and Lesser Caucasus and Nakhichevan

Didmanidze, 1979: Melitaea phoebe Den.et Schiff. ssp. caucasica, Turianchay Reserve; Nakhichevan, Ordubad;

neighborhood of Shakhbuz

Tuzov, 1993: Melitaea enoch Higgins, 1941, Armenian plateau

Tuzov, 1993: Melitaea phoebe ottonis Fruhstorfer, 1917, Armenian plateau; Talysh

Tuzov, 2000b: Melitaea (phoebe) pseudosibina Alberti, Talysh Mts., Zuvand Plateau, Gosmalyan

Tuzov, 2000b: Melitaea (phoebe) phoebe pttonis Fruhstorfer, Talysh Mts., Zuvand Plateau, Gosmalyan

Tschikolovets, Nekrutenko, 2012: Melitaea phoebe ottonis Fruhstorfer, 1917, Alty-Agatsh; Talysh-Zuvand, Kosmoljan; Nakhichevan, Bilav, Paragatshaj; Elisavetpol; Khatshmassky distr., Nabran" vill.

Korb, Bolshakov, 2016: Melitaea phoebe (Goeze, [1779]), Azerbaijan

Material: $10^{*}$, Nakhichevan, Agdere, 21.07.2017; 10", same place, 9.06.2017; $10^{*}, 1$, Zakatala, Djidjikhana, 10-12.07.2015

260. Melitaea ornata telona Fruhstorfer, 1908

Tuzov, 2000b: Melitaea (phoebe) punica amanica Rebel, Talysh Mts., Zuvand plateau, Gosmalyan

S.K.Korb, 2005: Melitaea punica amanica Rebel, Talysh

Tschikolovets, Nekrutenko, 2012: Melitaea ornata telona Fruhstorfer, 1908, Akstafa vic., Pojlu; Terter

Korb, Bolshakov, 2016: Melitaea ornata Christoph, 1893, Azerbaijan

Material: $1 \sigma^{\prime}$, Nakhichevan, Shakhbuz distr., between the village of Mahmudoba and Nursu, 30.05.2018

261. Melitaea cinxia cinxia (Linnaeus, 1758)

Ménétriés, 1832: Argynnis Cinxia, Linn., Talyche

Lederer, 1870: Meliteae Cinxia L., Talyche

Romanoff, 1884: Melitaea Cinxia L., Ordoubad

Christoph, 1886: Melitaea Cinxia L., Talysch

Effendi, 1970: Meliteae cinxia L., everywhere

Didmanidze, 1979: Meliteae cinxia L., Turianchay Reserve; road to Baku, the pass, the vicinity of the lake; Nakhichevan,

Ordubad, Bilav

Tuzov, 1993: Melitaea cinxia clarissa Staudinger, 1901, Armenian plateau; Talysh

Tschikolovets, Nekrutenko, 2012: Melitaea cinxia cinxia (Linnaeus, 1758), Turianchaj; Lerik; Talysh-Zuvand, Goveri; Alty-

Agach; Elisavetpol; Terter; Talysh, Lerik distr., Gosmolyan

Korb, Bolshakov, 2016: Melitaea cinxia (Linnaeus, 1758), Azerbaijan

Material: 20"0", Shemakha, Demirchi, 3-5.07.2017; 10", Shakhbuz, Nursu, 8.06.2017; 10", Shemakha, Pyrguli, 25.06.2015; 10", 1\%, Shemakha, Archiiman-Pirguli, near Shakhdag Milli Park, 19-20.05.2013; 1\%, Nakhichevan, Agdere, Ordubad, 19.06 .2019 
262. Melitaea trivia roberti Butler, 1880

Romanoff, 1884: Melitaea Trivia Schiff., Ordoubad

Lederer, 1864: Melitaea trivia v. fascelis Esp., Helenendorf bei Elisabethpol

Effendi, 1970: Melitaea trivia Schiff., everywhere up to 1700-1900 m a.s.l.

Didmanidze, 1979: Melitaea trivia Den. et Shiff. v. pseudodidyma Rebel, Nakhichevan, Ordubad

Tuzov, 1993: Melitaea trivia Staudinger, 1861 (= kaschtschenkoi Christoph, 1889), Armenian plateau; Talysh

Tuzov, 2000e: Melitaea trivia caucasi Verity, Talysh Mts., Zuvand Plateau; Nakhichevan, Kyukyu

Didmanidze, 2004: Melitaea trivia Denis et Shiffermüller, 1775, Nakhichevan, Ordubad

Tschikolovets, Nekrutenko, 2012: Melitaea trivia roberti Butler, 1880, Palan-Tiukan, Kirobobad prope

Korb, Bolshakov, 2016: Melitaea trivia ([Denis et Schiffermüller], 1775), Azerbaijan

\section{Melitaea arduinna arduinna (Esper, [1783])}

Tuzov, 1993: Meliteae arduinna rhodopensis Freyer, 1836, Armenian plateau; Talysh

Tschikolovets, Nekrutenko, 2012: Melitaea arduinna arduinna (Esper, [1783]), Elisavetpol

Korb, Bolshakov, 2016: Melitaea arduinna (Esper, [1784]), Azerbaijan

Material: 19, Zakatala-Balaken, 1-4.04.2014

264. Argynnis paphia paphia (Linnaeus, 1758)

Subfamily Argynninae Duponchel, [1835]

Ménétriés, 1832: Argynnis Paphia, Linn., Talyche

Becker, 1873: Argynnis Paphia, Lenkoran

Christoph, 1874: Argynnis Paphia L., Lenkoran

Romanoff, 1884: Argynnis Paphia L., Lenkoran

Christoff, 1886: Argynnis Paphia L., Lenkoran

Jachontov, 1911: Argynnis paphia L., Ordubad; Lenkoran

Effendi, 1970: Argynnis paphia L., everywhere in the foothills and mountains

Didmanidze, 1979: Argynnis paphia L., дорога в Баку, перевал

Tuzov, 1993: Argynnis paphia masandarensis Gross et Ebert, 1975, Armenian plateau; Talysh

Tschikolovets, Nekrutenko, 2012: Argynnis paphia paphia (Linnaeus, 1758), Elisavetpol; Adzhi-kent

Korb, Bolshakov, 2016: Argynnis paphia(Linnaeus, 1758), Azerbaijan

Material: 1\%, Shemakha, Demirchi, 3-5.07.2017; 19, Shemakha, Pirguli, 28.06.2013; 30'0', 19, Gabala, near Vendam and Bunud, 5-8.07.2016; 19, Lenkoran, Dashtatyuk, 16.06.2016; 10', Zakatala-Balaken, 1-4.04.2014; 10', Zakatala, from kombinat to State Reserve, 24.06.2014; 10", Belokani, Beshbulag, Djidjikhana, 25.06.2013; 30'0', 2\%o, Lenkoran, Azfilial, 2528.05.2013; 10', 19, Ismailly Kaladjik, Velasin, 21-23.07.2014; 20º', Goygol, Togana, 24-25.07.2019

265. Argynnis laodice laodice (Pallas, 1771)

Romanoff, 1884: Argynnis Laodice Pall., Lenkoran

Christoph, 1886: Argynnis Laodice Pall., Lenkoran

Elwes, 1889: Argynnis Laodice Pall., Lenkoran

Heyne, [1895]: Argynnis Laodice Pall., Lenkoran

Effendi, 1970: Argynnis laodice Pall., Talysh, Sim (Sım)

266. Argynnis niobe taura Röber, 1896

Lederer, 1870: Argynnis Niobe L., Talyche

Romanoff, 1884: Argynnis Niobe L. var. Eris Meig., Ordoubad

Christoph, 1886: Argynnis Niobe L., Lenkoran

Effendi, 1970: Argynnis (Fabriciona) niobe L. (= ssp. taurica Röber; ssp. orientalis Alph.), subalpine zone of the Greater and Lesser Caucasus, Avakhyl, Laza, Ryuk, Yanshah, Agdavan, Yerfi; Nakhichevan, Gjarmachaty, Pais; Talysh, Kelvyaz,

Kyalahan

Didmanidze, 1979: Argynnis niobe L. sbsp. eris Meg., Kaspiyskayaı Nakhichevanç Ordubad

Tuzov, 1993: Fabriciana niobe orgova Teich, 1901, Armenian plateau

Tuzov, 1993: Fabriciana niobe demavendis Gross et Ebert, Talysh

Tuzov, 2000b: Argynnis niobe gigantea Staudinger, Nakhichevan, Pazmara, Buzgov, near Ordubad

Tuzov, 2003: Argynnis niobe ssp. gigantea Staudinger, 1871 (= demavendis Gross \& Ebert, 1975), Nakhichevan

S.K.Korb, 2005: Argynnis niobe demavendis Gross \& Ebert, 1975, Talysh

Tschikolovets, Nekrutenko, 2012: Argynnis niobe taura Röber, 1896, Adzhi-kent; Elisavetpol; Talysh, Lerik

Korb, Bolshakov, 2016: Argynnis niobe (Linnaeus, 1758), Azerbaijan

Material: 20'0', Shakhbuz, Nursu, 8.06.2017

267. Argynnis adippe adippe ([Dennis \& Schiffermüller], 1775)

Christoph, 1886: Argynnis Adippe L. ab. Cleodoxa O., Talysch 
Jachontov, 1911: Argynnis adippe L. var. (subsp.) thalestria Jach., Baku; Kusary

Effendi, 1970: Argynnis (Fabriciona) addipe L. (= ssp. thalestria Jach.), everywhere in the mountains

Tuzov, 1993: Fabriciana adippe thalestria Jachontov, 1908, Armenian plateau; Talysh

Tuzov, 2000c: Argynnis adippe ([Dennis \& Schiffermüller], 1775), Armenian Highland

Tuzov, 2000c: Argynnis adippe ssp. taurica Staudinger, 1878, Armenian Highland

Tschikolovets, Nekrutenko, 2012: Argynnis adippe adippe ([Dennis \& Schiffermüller], 1775), Alty-Agatsh; Talysh, Lerik

Korb, Bolshakov, 2016: A rgynnis adippe (Linnaeus, 1767), Azerbaijan

268. Argynnis aglaja aglaja (Linnaeus, 1758)

Effendi, 1970: Argynnis aglaja L. ssp. ottomana Rober, everywhere

Tuzov, 1993: Mesoacidalia aglaja ottoman Rober, 1896, Armenian plateau; Talysh

Tuzov, 2000c: Argynnis aglaja ssp. ottomana Röber, 1896, Armenian Highland, Talysh

Tschikolovets, Nekrutenko, 2012: Argynnis aglaja ag/aja (Linnaeus, 1758), Elisavetpol; Karmalinovka, Nachitschevan

Korb, Bolshakov, 2016: Argynnis aglaja (Linnaeus, 1758), Azerbaijan

Material: 10, Nakhichevan, Shakhbuz, Bichenek, Shakhbuz National Park, 26.07.2016; $10^{\prime \prime}$, Nakhichevan, Ordubad, Agdere,

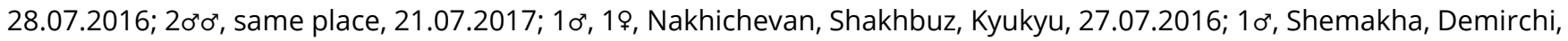
3-5.07.2017; 20'0', Kedabek, Novosaratovka, Novoivanovka, 21.06.2014

269. Argynnis alexandra alexandra Ménétriés, 1832

Ménétriés, 1832: Argynnis Alexandra, mihi, Zouvant, Talyche

Kolenati, 1846: Argynnis Alexandra, Ménétr., Talysch

Eversmann, 1851: Argynnis Alexandra Men., Talyche

Nordmann, 1851: Argynnis Alexandra, Ménétr., Talysch

Ménétriés, 1855: Argynnis Alexandra, Lenkoran, M.Taliisch

Lederer, 1870: Argynnis Alexandra Mén., Zouvent

Christoph, 1874: Argynnis Alexandra Mén., Lenkoran

Romanoff, 1884: Argynnis Alexandra Mén., Lenkoran

Christoph, 1886: Argynnis Alexandra Mén., Lenkoran, Suant

Bramson, 1890: Argynnis Alexandra Mén., Lenkoran

Effendi, 1970: Argynnis alexandra Men., Talysh, Kosmolyan; Konakhkend, Ryuk; Babadag Mt.

Tuzov, 1993: Mesoacidalia alexandrs Mentries, 1832, Talysh

Tuzov, 2000c: Argynnia alexandra Ménétriés, 1832, Talysh

Tuzov, 2000c: Argynnia alexandra alexandra Ménétriés, 1832, Talysh

Tuzov, 2000b: Argynnia alexandra Ménétriés, 1832, Talysh Mts., Zuvand Plateau, Dzhoni

Tuzov, 2003: Argynnia alexandra Ménétriés, 1832, Talysh

Tschikolovets, Nekrutenko, 2012: Argynnia alexandra alexandra Ménétriés, 1832, Talysh, Orantshay; Masally vill.; Talysh, Lerik; Talysh, Allar

Korb, Bolshakov, 2016: Argynnis alexandra Ménétriès, 1832, Azerbaijan

Material: 1 ơ, Nakhichevan, Shakhbuz, Bichenek, Shakhbuz National Park, 26.07.2016

270. Argynnis pandora pandora ([Denis \& Schiffermüller], 1775)

Ménétriés, 1832: Argynnis Cynara, Fa., Talyche

Kolenati, 1846: Argynnis Pandora. Esp. Cynara. Fabr., Talysch

Lederer, 1870: Argynnis PandoraS. V., Talyche

Christoph, 1874: Argynnis Pandora Schiff., Lenkoran

Romanoff, 1884: Argynnis Pandora Schiff., Ordoubad; Akstafa

Christoph, 1886: Argynnis Pandora Schiff., Lenkoran

Heyne, [1893]: Argynnis Pandora Schiff., Lenkoran

Effendi, 1970: Argynnis pandora Schiff., everywhere in the foothills, rarely in the lowlands

Didmanidze, 1979: Argynnis Pandora Den. et Schiff., Nakhichevan, Ordubad; Djulfa, geyser; Paragachai river gorge

Tuzov, 1993: Argynnis Pandora transcaucasica Moucha, 1967, Armenian plateau; Talysh

Tuzov, 2000b: Argynnis pandora pandora Denis \& Schiffermüller, Nakhichevan, Pass Bichenek

Didmanidze, 2004: Argynnis pandora Denis \& Schiffermüller, 1775, Nakhichvan, Julfa; Paragachai

Tschikolovets, Nekrutenko, 2012: Argynnis pandora pandora ([Denis \& Schiffermüller], 1775), Elisavetpol; Terter Korb, Bolshakov, 2016: Argynnis pandora ([Denis et Schiffermüller], 1775), Azerbaijan

Material: $10^{\prime \prime}, 19$, Nakhichevan, Shakhbuz, Bichenek, Shakhbuz National Park, 26.07.2016; 20"0", 2\%ᄋ, same place; 10",

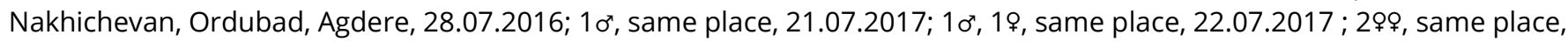

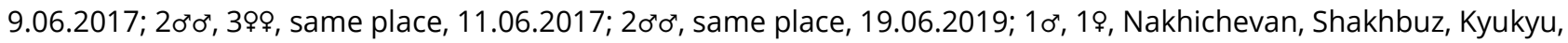
27.07.2016; 2\%o, Nakhichevan, Shakhbuz distr., between the village of Mahmudoba and Nursu, 30.05.2018; 19, Lerik, Gosmalyan, 21-22.06.2017; 19, Kuba, Afurdja, 8.07,2019 
271. Brenthis hecate transcaucasica Wnukowsky, 1929

Lederer, 1864: Argynnis Hecate S. V., Helenendorf bei Elisabethpol

Effendi, 1970: Argynnis (Brenthis) Hecate Esp. ssp. caucasica Stgr., Nakhichevan, Ordubad, Djuga, Norashen (Sharur)

Didmanidze, 1979: Brenthis hecate Den. et Shiff., Turianchai reservation; road to Baku

Tuzov, 1993: Brenthis hecate transcaucasica Wnukowsky, 1929, Armenian plateau; Talysh

Tschikolovets, Nekrutenko, 2012: Brenthis hecate transcaucasica Wnukowsky, 1929, Bitshenek, lac.Batabat; Adzhi-kent;

Elisavetpol; Turantshaysky nature reserve; Alty-Agatsh

Korb, Bolshakov, 2016: Brenthis hecate ([Denis et Schiffermüller], 1775), Azerbaijan

Material: 20'0", Shemakha, Pirguli, 28.06.2013

272. Brenthis ino ino (Rottemburg, 1775)

Effendi, 1970: Argynnis (Brenthis) ino Rott., Shemakha, Pirkuli; Vartashen (Oguz); yardimli, Chayuzi; Nakhichevan,

Shakhbuz

Tuzov, 1993: Brenthis ino, Armenian plateau, Talysh

Tuzov, 2000c: Brenthis ino ssp.schmitziWagener, 1983, Armenian Highland; Talysh

Tschikolovets, Nekrutenko, 2012: Brenthis ino ino(Rottemburg, 1775), Bitshenek, lac.Batabat

Korb, Bolshakov, 2016: Brenthis ino (Rottemburg, 1775), Azerbaijan

273. Brenthis daphne daphne (Bergsträsser, 1780)

Ménétriés, 1832: Argynnis Daphne, Fab., Talyche

Lederer, 1864: Argynnis daphneS. V., Helenendorf bei Elisabethpol

Christoph, 1877: Argynnis Daphne Schiff, Lenkoran

Romanoff, 1884: Argynnis Daphne Schiff, Lenkoran

Christoph, 1886: Argynnis Daphne Schiff, Talysch, Lenkoran

Effendi, 1970: Argynnis (Brenthis) daphne Schiff., Shemakha, Kirovka, Avakhil; Lagich, Aterk; Shusha; Lachin, Yanshakh

Didmanidze, 1979: Brenthis daphne Denn et Schiff., Turianchai reservation, road to Baku, Nizovaya

Tuzov, 1993: Brenthis daphne ssp., Armenian plateau; Talysh

Didmanidze, 2004: Brenthis daphne Denis et Schiffermüller, 1775, Turianchai Reservation; Nizovaya, Baku distr.

Tschikolovets, Nekrutenko, 2012: Brenthis daphne daphne (Bergsträsser, 1780), Elisavetpol; Tterter (gub.Elisavetpol)

Korb, Bolshakov, 2016: Brenthis daphne (Bergsträsser, 1780), Azerbaijan

Material: 1ơ, 1\%, Shemakha, Pirkuli, 28.06.2013

274. Issoria lathonia lathonia (Linnaeus, 1758)

Ménétriés, 1832: Argynnis Lathonia, Linn., Lenkoran

Lederer, 1870a: Argynnis Lathonia, Linn., Talyche

Christoph, 1886: Argynnis Lathonia, Linn., Lenkorn

Effendi, 1970: Argynnis (Isoria) lathonia L., everywhere

Didmanidze, 1979: Argynnis lathonia Brgstr., Turianchai reservarion; Pirkuli reservation, Dyubrar ridge, Pirsagat river gorge; Nakhichevan, Djulfa, geyser; Kyukyu neighborhood, Paragachay river gorge, Bilav

Tuzov, 1993: Issoria lathonia lathonia Linnaeus, 1758, Armenian plateau; Talysh

Tschikolovets, Nekrutenko, 2012: Issoria lathonia lathonia (Linnaeus, 1758), Terter (gub.Elisavetpol; Adzhi-kent

Korb, Bolshakov, 2016: Issoria lathonia (Linnaeus, 1758), Azerbaijan

Material: $1 \sigma^{\prime \prime}$, Nakhichevan, Shakhbuz, Nursu, 7.05.2018; 40" $0^{\prime \prime}$, Nakhichevan, Shakhbuz distr., between the village of Mahmudoba and Nursu, 30.05.2018; $10^{\prime \prime}$, Gabala, near Vendam and Bunud, 5-8.07.2016; 10", Yardimli, 17-20.05.2016; 10",

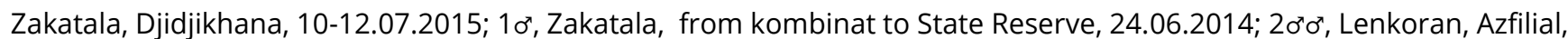
16-19.09.2013; 10', Ismailly Kaladjik, Velasin, 21-23.07.2014

\section{Clossiana eunomia eunomia (Esper, [1799])}

Ménétriés, 1832: Argynnis Aphirape, Hubn., Talyche

Lederer, 1870: Argynnis Aphirape Hb., Talyche

Effendi, 1970: Argynnis (Brenthis) aphirape HB, Shemakha, Chukhuryurd (Çuxuryurd); I Talysh, Sarak

276. Clossiana selene selene ([Dennis \& Schiffermüller], 1775)

Effendi, 1970: Argynnis selene Schiff., everywhere in the foothills, rarely in the lowlands

277. Boloria euphrosyne euphrosyne (Linnaeus, 1758)

Tschikolovets, Nekrutenko, 2012: Clossiana euphrosyne euphrosyne (Linnaeus, 1758), Alty-Agatsh

Korb, Bolshakov, 2016: Boloria euphrosyne (Linnaeus, 1758), Azerbaijan

Material: 20'0', 2\%o, Zakatala State Reserve, Akhkemal, 11.06.2015 
278. Boloria dia dia (Linnaeus, 1767)

Romanoff, 1884: Argynnis Dia L., Hankynda

Jachontov, 1911: Argynnis dia L. var. calida n., Zakataly, Kakhi vill.; Poylu vill.

Tschikolovets, Nekrutenko, 2012: Clossiana dia dia (Linnaeus, 1767), Elisavetpol; Terter

Korb, Bolshakov, 2016: Boloria dia (Linnaeus, 1767), Azerbaijan

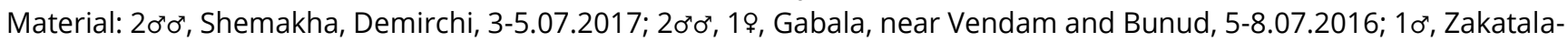
Balaken, 1-4.04.2014; 10", Shemakha, Pyrguli, 25.06.2015; $10^{\circ}$, same place, 28.06.2013; 10', Zakatala, Djidjikhana, 10-

12.07.2015; 30'0 ', Kuba, Afurdja, 8.07,2019

279. Boloria caucasica caucasica (Lederer, 1852)

Effendi, 1970: Argynnis (Brenthis) pales Schiff. ssp. caucasica Stgr.(Brenthis) dia L., Shakhdag, Bazardyuzi, Babadag Mts. (2800-3100 M); Kapudjik, Koshkardag Mts. (2800-2900 m a.s.I.)

Tschikolovets, Nekrutenko, 2012: Boloria caucasica caucasica (Lederer, 1852), ms Kjapaz (prope Elisavetpol); Murov-dagh (gub. Elisavetpol)

Korb, Bolshakov, 2016: Boloria caucasica (Lederer, 1853), Azerbaijan

\section{Boloria titania Esper, 1793}

Effendi, 1970: Argynnis (Brenthis) amathusia Esp., Kutkashen (Gabala), Kyamyarvan (Kəmərvan)

\section{Conclusions}

As a result of the work conducted a list of the total species composition of butterflies in Azerbaijan, which currently includes 280 species from 6 families (Hesperiidae - 37, Papilionidae - 10, Pieridae - 28, Lycaenidae - 100, Riodinidae - 1, Nimphalidae -104) for today was compiled. We record Papilio demoleus demoleus Linnaeus, 1758 as a new species for the fauna of the Caucasus. The given data will help to increase species richness and expand the information on the distribution of the butterflies (Papilionoformes) in Azerbaijan and the Caucasus as a whole and will be useful for nature conservation institutions, reserves and wildlife sanctuaries.

\section{Acknowledgments}

We express special thanks to our colleague and friend Dr. I. G. Kerimova for help in translating the article into English.

\section{References}

Alphéraky, S.N. (1876). Die Lepidopteren des nördlichen Kaukasus. Trudy russk. net. Obshch. 10, 3-34 (Russian) Alphéraky, S.N. (1910). Matériaux scientiques. Revue Russe Ent., 9(4), 347-375 (Russian)

Back, W., Knebelsberger, Th., Miller, M., (2008). Molecularbiologische Untersuchungen und Systematik der palaearktischen Arten von Euchloe Hübner, [1819] (Lepidoptera: Pieridae). Ent. Zt., 118(4), 151-169.

Bálint, Zs. (1993). Egy xeromontán boglárkalepke: a Plebejus pylaon (Fischer von Waldheim, 1832) és rokonsági köre (Lepidoptera, Lycaenidae), III. A csoport filogenetikája és állatföldrajza. Jan. Pann. Múz. Pécs (1992) 37, 45-64 (Hungarian)

Bálint, Zs., Kertész, A., Lukhtanov, V.A. (1993). A survey of the Subgenus Plebejides Sauter, 1968 (Lepidoptera, Lycaenidae). Ent. Obozr., 71(4), 863-886 (Russian)

Becker, A. (1873). Reise nach Baku, Lenkoran, Derbent, Madschelis, Kasum Kent, Achty. Bull. Soc. Nat. Mosc. 46, $229-258$. Bogdanov, P.V. (2000). Family Lycaenidae: Aricia, Pseudoaricia, Ultroaricia. In: Giide to the Butterflies of Russia and Adjacent Territories (Lepidoptera, Rhopalocera). Pensoft, Sofia-Moscow, 2, 172-175.

Bogdanov, P.V. (2007). Classification of the Satyrid Butterflies of the genus Pseudochazara de Lese, 1951 (Lepidoptera, Satyridae) of Europe, Northern Africa and Asia Minor. Trudy Gos. Darvinovskogo muzeya, 10, $136-205$ (Russian).

Bogdanov, P.V. (2008). Classification of the Satyrid Butterflies of the genus Satyrus Latreille, 1819 (Lepidoptera, Satyridae) of Palaearctic. Trudy Gos.Darvinovskogo muzeya, 12, 174-251 (Russian).

Bogdanov, P.V., Samodurov, G.D., Tuzov, V.K. (1997). Family Satyridae. In: Guide to the Butterflies of Russia and Adjacent Territories (Lepidoptera, Rhopalocera). Pensoft, Sofia-Moscow, 1, 183-260.

Bollino, M., Sala, G. (2004). Synops of Papilio alexanor Esper, 1799. (An updated revue of its taxonomy and biology). Nature Edizioni Scientifiche, Bologna, 1-60.

Bollow, C. (1930). Pieridae. In: Seitz, A.Die Gross-Schmetterlinge der Erde. Die Palaearkischen Tagfalter. Upplement. Stuttgart, Alfred Kernen Vrlg., 1, 93-125.

Bozano, G. (2002). Satyrinae. Part III. Tribe Satyrini, Subtribes Melanargiina and Coenonymphina. In: Bozano, G.C. (Ed.) Guide to the Butterflies of the Palearctic Region. Milano, Omnes Artes, 4, 1-71.

Bozano, G.C., Weidenhoffer, Z. (2007). Lycaenidae. Part III. Subfamly Theclinae, Tribes Tomarini, Aphnaeini and Theclini (partim). In: Bozano, G.C. (Ed.) Guide to the Butterflies of the Palearctic Region. Milano, Omnes Artes, 9, 1-97.

Bramson, K.L. (1890). Die Tagfalter (Rhopalocera) Europa"s und des Caucasus. Analytisch bearbeitet. Kiew, Selbstverlag. 
Bryk, F., Eisner, C. (1932). Kritische revision der Gattung Parnassius unter Benutzung des materials der Kollektion Eisner, Dahlem. Parnassiana, 2(3), 34-42.

Carbonell, F. (1994). Contribution à la connaissance du genre Polyommatus Latreille, 1804: le complexe ultraspécifique de Polyommatus eros-eroides au Moyen-Orient et en transcaucasie (Lepidoptera: Lycaenidae) (2ème partie: diagnose et discussion). Linn. Belgica, 14 (6), 439-454.

Carbnell, F., Borie J.P., De Prins, J. (2004). Une nouvelle espèce du genre Turanana du Pakistan (Lepidoptera: Lycaenidae). Phegea, 32(3), 77-90.

Christoph, H. (1874). Weiterer Beitrag zum Verzeichnisse der in Nord-Persien einheimischen Schmetterling. Horae Sco. ent. Ross., 10(1), 3-55.

Christoph, H. (1877). Sammelergebnisse aus Nordpersien, Krasnowodsk in Turkmenien und dem Daghestan. Horae Societatis entomologicae Rossicae, 12, 181-299.

Christoph, H. (1886). Verzeichniss aller bisjetzt in Talysch gesammelten Schmetterlinge. In: Radde, G. Fauna und Flora des südwestlichen Caspiengebietes, Leipzig, 236-245.

Chrystoph, H. (1889). Neue Lepidopteren aus dem Kaukasus. In: Romanoff, N.M. (Réd.). Mémoires sur les Lépidoptères, St.-Pétersbourg, 5, 193-202.

Christoph, H. (1893). Lepidoptera Nova Faunae Palaearcticae. Dt. ent. Ztg. Iris, 6, 86-96.

D"Abrera, B. (1993). Butterflies of the Holarctic Region, Part 3. Nymphalidae (part), Libytheidae, Riodinidae \& Lycaenidae. Hill House, Melbourne.

Dantchenko, A.V. (2000). Family Lycaenidae (part). In: Guide to the Butterflies of Russia and Adjacent Territories (Lepidoptera, Rhopalocera). Pensoft, Sofia-Moscow, 2, 85-101, 186-189, 196-214.

Dantchenko, A.V. (2004). A new species of the genus Agrodiaetus Hübner, [1822] from Transcaucasia (Lepidoptera, Lycaenidae). Atalanta, Würzburg, 35(3/4), 323-326.

Dantchenko, A.V., Lukhtanov, V.A. (1994). New taxa of the subgenus Agrodiaetus Hübner, 1822 from Caucasus (Lepidoptera, Lycaenidae). Atalanta, Würzburg, 25(1/2), 207-213.

Dantchenko, A.V., Lukhtanov, V.A. (2004). New taxa of the "brown" species-copmlex of the genus Agrodiaetus Hübner, [1822] from Transcaucasia (Lepidoptera, Lycaenidae). Atalanta, Würzburg, 35(3/4), 327-334.

Davenport, D. (1941). The butterflies of the satyrid genus Coenonympha. Bull. Mus. comp. Zool. Harv, 87(4), 213-349.

De Freina, J.J. (1983). Studien über Biologie, Verbreitung, geographische Variabilität und Morphologie von Gonepteryx farinosa (Zeller, 1847) nebst zusätzlicher Erläuterung der Verbreitung und geographischen Variabilitvt von Gonepteryx rhamni (Linné, 1758) in Kleinasien (Lepidoptera, Pieridae). Mitt. münch. ent, Ges. 72, 9-55.

De Freina, J.J., Aussem, B. (1987). Chazara bischoffi (Herrich-Schäffer, 1846) und ihre nächstverwandten Arten (Lepidoptera, Satyridae). NachrBl. bayer. Ent. 36(1), 1-10.

De Jong, R. (1974). Sytematics and evolution of the Palaearctic Spialia species (Lepidoptera, Hesperiidae). Tijdschr. Ent., $117(6), 225-271$.

Devyatkin, A.L. (1988). Taxonomical notes of the genus Carcharodus (Lepidoptera, Hesperiidae). Vest. Zool, (1), 40-44 (Russian).

Devyatkin, A.L. (1990). To the systematics of skippers of Muschampia poggei group (Lepidoptera, Hesperiidae). Zoologicheskiy Zhurnal, 69 (9), 51-56 [in Russian]

Devyatkin, A.L. (1994). Distribution and variatioan of Pyrgus jupei (Alberti, 1967) (Lepidoptera, Hesperidae), with discussion of the taxonomic status of Pyrgus centralasiae Renner, 1991. Actias, 1(1/2), 5-9.

Devyatkin, A.L. (1997). Hesperiidae. In: Guide to the Butterflies of Russia and Adjacent Territories (Lepidoptera, Rhopalocera). Pensoft, Sofia-Moscow, 105-133.

Devyatkin, A.L. (2000). Family Nymphalidae: Mellicta. In: Guide to the Butterflies of Russia and Adjacent Territories (Lepidoptera, Rhopalocera). Pensoft, Sofia-Moscow 2, 76-82.

Didmanidze, E.A. (1979). Lepidoptera of arid regions of the Transcaucasia. Tbilisi, 43-114 (Russian).

Didmanidze, E.A. (2004). Anotated list of Diurnal Butterflies (Lepidoptera: Rhopalocera) of Georgia and adjacent territory from Southern Caucasus. Proc. Inst. Zool. Georgia, Tbilisi, 197-226.

Eckweiler, W. (2002). Zwei neue Unterarten des Subgenus Agrodiaetus Hübner, 1822 aus Nordwest-Iran (Lepidoptera: Lycaenidae, Gattung Polyommatus). Nachr. ent. Ver. Apollo Frankfurt/man, N.F. 23(1/2), 77-79.

Eckweiler, W. (2003). Eine neue Unterart von Colias Chlorocoma Christoph, 1988 aus dem iranischen Talesh (Lepidoptera: Pieridae). Nachr. ent. Ver. Apollo, 24(3), 147-152.

Eckweiler, W., Bozano, G.C. (2011). Guide to the butterflies of the Palaearctic region. Satyrinae Part IV. Omnes Artes, Milano.

Eckweiler, W., Häuser, C.L. (1997). An illustrated checklist of Agrodiaetus Hübner, 1822, a subgenus of Polyommatus Latreille, 1804 (Lepidoptera: Lycaenidae). Nachrichten des entomologischen Vereins Apollo, Suppl. 16, $113-168$.

Eckweiler, W., Hofmann, P. (1980). Verseichnis iranischer Tagfalter. Nachr. ent. Ver. Apollo, Frankfurt/Main, N.F., Suppl., 1, $1-27$.

Eckweiler, W., ten Hagen, W. (1998). Zur Taxonomie von Polyommatus (Agrodieaetus) phyllus (Christoph, 1877), P.posthumus (Christoph, 1877) und P.darius spec. nov. (Lepidoptera: Lycaenidae). Nachr. ent. Ver. Apollo, Frankfurt/Main, N.F., 19, 109-118. 
Eitschberger, U., Steiniger, H. (1975). Die geographische Variation von Eumedonia eumedon (Esper, 1780) in der westlichen Palaearktis (Lep. Lycaenidae). Atalanta, Würzburg 6, 84-125.

Effendi, R.M.-E. (1970). Visshie cheshuekrilie Azerbaidjana, ikh biologiya, ekologiya, zoogeografiya I khozaystvennoe znachenie (bez semeystv Noctuidae i Geometridae). The highest Lepidoptera of Azerbaijan, their biology, ecology, zoogeography and economic importance (without families Noctuidae and Geometridae). Kandidatskaya disserracuya, Baku, 1-389.

Elwes, H.J. (1889). A revision of the genus Argynnis. Trans. ent. Soc. Lond., 37(4), 535-575. https://doi.org/10.1111/j.13652311.1889.tb00934.x

Evans, W.H. (1949). A catalogue of the Hesperidae from Europe, Asia und Australia in the British Museum (Natural History). Brit. Mus. (Nat. Hist.), London. https://doi.org/10.5962/bhl.title.105941

Eversmann, E. (1848). Beschreibung einiger neuen Falter Russlands. Bull. Soc. Nat. Moscou 21(3), 205-232.

Forster, W. (1938). Die Lycaena pylaon-Gruppe. Ent. Runds., 55(21), 213-219; 55(22), 236-239; 55(29), 334-337; 55(31), 361364; 55(36), 417-420; 55(42), 485-490.

Forster, W. (1956). Bausteine zur Kenntnis der Gattung Agrodiaetus Scudd. (Lep.Lycaen.) I. Z. wien. ent. Ges., 41, 42-61, 7089, 118-127.

Forster, W. (1960). Agrodiaetus rjabovisp. nov. Ent. Zeit., 70(14), 1-2.

Forster, W. (1961). Bausteine zur Kenntnis der Gattung Agrodiaetus Scudd. (lep.Lycaen.) II. Z. Wien. Ent. Ges., 46, $110-116$. Fruhstorfer, H. (1918). Neue paläarktische Rhopalocera. Ent. Zt., 31(21), 81-82.

Gaede, M. (1930). Satyridae, Nymphalidae, Erycinidae, Lycaenidae. In: Seitz. A. Die Gross-Schmetterlinge der Erde. Die Palaearktischen Tagfalter. Supplement. Stuttgart, Alfred Kernen Vrlg., 1, 131-249.

Gaede, M. (1931). Satyridae. I-III. In: Lepidopterorum Catalogus ed. E. Strand. W. Junk., Berlin 43, 1-320; 46, 321-544; 48, 545-759.

Gerhard, B. (1853). Versucheiner Monograhie der europäischen Schmetterlingsarten: Thecla, Polyommatus [sic], Lycaena, Nebeobius. Als Beitrag zur Schmetterlingskunde. Hamburg bei Herausgeber, Leipzig, W. Gerhard.

Gorbunov, P.Y. (2001). The Butterflies of Russia: classification, Genitalia, keys for identification (Lepidoptera: Hesperioidea and papilionoidea). "Thesis" Ekaterinburg.

Häuser, C.L. (1982). Zur Verbreitung, Biologie und Taxonomie von Ochlodes hyrcanus (Christoph, 1893) (Hesperidae). Nota lipid, 5(2/3), 86-102.

Hemming, A.F. (1929). Revision of the baton group of the enus Turanana Betune-Baker, with an account of an unrecognized species, T. vicrama Moore. Entomologists, 62, 27-34, 60-64, 84-89.

Hemming, A.F. (1931). On the dates of Publication of F.Ruhl"s. "Die palaearktischen Grossschmetterlinge und ihre naturgescfichte". Ann. Mag. nat. Hist., Ser., 8(10), 405-406. https://doi.org/10.1080/00222933108673411

Hesselbarth, G., van Oorschot, H., Wagener, S. (1995). Die Tagfalter der Türkei unter Berücksichtigung der angrenzenden Länder. Selbstverlag Sigbert Wagener, Bocholt, 1354 S.

Heyne, A. ([1893-1895]). Tagfalter. In: Rühl, F., Heyne, A., Die palaearktischen Grossschmetterlinge und ibre Naturgeschichte. Leipzig, E. Heyne, 1, 385-688.

Heyne, A. (1895). Nachträge und Berichtigungen, [Tagfalter\}. In: Rühl, F., Heyne, A., Die palaearktischen Grossschmetterlinge und ibre Naturgeschichte. Leipzig, E. Heyne, 1,689-857.

Higgins, L.G. (1941). An illusttated catalogue of the palearctic Melitaea (Lep.Rhopalocera). Trans. Ent. Sco. Lond., 91(7), 175-365. https://doi.org/10.1111/j.1365-2311.1941.tb01045.x

Ilyina, E.V. (2006). The new interesting records of Lepidoptera in Dagestan. In: Materualy VII mezhdunarodnoy konferentsii "Biologicheskoe raznoobrazie Kavkaza". Nalchik, 306-307 (Russian).

Jachontov, A.A. (1911). Bemerkungen über die Rhoppalocera des Kaukasus nach dem material des kaukasischen Museums Tiflis. Izv. Kavkaz. Muz., 5(4), 291-316 (Russian).

Jachontov, A.A. (1913). Quelques mots sur Chiladesphiala Gr.-Gr. (Lepidoptera, Lycaenidae). Russ. ent. obozr., 13(1), 110112 (Russian).

Kaabak, L.V., Tarasov, E.A., Tuzov, V.K. (1997). Family Papilionidae. In: Guide to the Butterflies of Russia and Adkacent Territories (Lepidoptera, Rhopalocera). Pensoft, Sofia-Moscow, 1, 135-151.

Koçak, A.Ö. (1996). List of the Taxa of Polyommtus of the South West Asia, with Some Nomenclatural Notes (Lepidoptera, Lycaenidae). Misc. Papers, 30/33, 1-32 (Turkish).

Kolenati, F.A. (1846). Meletemata entomologica. Insecta Caucasi: Coleoptera, Dermaptera, Lepidoptera, Neuroptera, Mutillidae, Aphaniptera, Anoplura. Ptropoli, Typis Imp. Acad. Sci., 5, 3-170.

Korb, M. (1920). Über die von mir beobachteten paläarktischen Lepidopteren (Vorkommen, Lebensweise usw.). Mitt. münch. ent. Ges., 9, 57-64.

Korb, S.K. (2005). A catalogue of butterflies of the ex-USSR, with remarks on systematics and nomenclature. Korb press. N. Novgorod.

Korshunov, Y.P. (1972). A catalogue of butterflies (Lepidoptera, Rhopalocera) of the USSR fauna. Entomologicheskoe obozreniye, 51(1), 136-154; 51(2), 352-367 (in Russian).

Korshunov, Y.P. \& Dubatolov, V.V. (1987). New for the USSR fauna species and subspecies of butterflies. Vestnik zoologii, (3), 87 (in Russian).

Kudrna, O. (1977). A revision of the genus Hipparchia Fabricius. Oxon, Farington. 
Lederer, J. (1864). Zur Lepidopteren-Fauna von Imeretien und Grusien. Wien. ent. Monatschr., 8(5), 165-172.

Lederer, J. (1870). Contributions à la faune des Lépidoptères de la Transcaucasie. Annl. Sco. ent. Belg., $13,17-54$.

Lukhtanov, V.A. (1987). A review of palaearctical butterflies of the genus Oeneis Hübner (Lepidoptera, Satyridae). II. O. jutta species group. Entomologicheskoe obozreniye, 66(2), 142-158 (in Russian).

Lukhtanov, V.A. (1989). A review of the palaearctic satyrids of the group Oeneis norna (Lepidoptera, Satyridae). Vestnik zoologii, (2), 28-36 (in Russian).

Lukhtanov, V.A. (2000). Zur Systematik und Verbreitung der Taxa der Athamanthia dimorpha-Gruppe (Lepidoptera, Lycaenidae). Atalanta, 31 (1/2), 179-192.

Lukhtanov, V. \& Lukhtanov, A. (1994). Die Tagfalter Nordwestasiens (Lepidoptera, Diurna). Herbipoliana, 3, 1-440.

Lukhtanov, V.A., Kuznetsova, V.G. (1989). The analysis of the karyotype variability in the butterflies of the Melitaea didyma group along with evidence of the species distinctness of M. latonigena (Lepidoptera, Nymphalidae). Zool. Zh., 68(12), 3846.

Lukhtanov, V.A., Dantchenko A.V., Vishnevskaya M.S., Saifitdinova A.F. (2015). Detecting cryptic species in sympatry and allopatry: analysis of hidden diversity in Polyommatus (Agrodiaetus) butterflies (Lepidoptera: Lycaenidae). Biological Journal of the Linnean Society, 116, 468-485. https://doi.org/10.1111/bij.12596

Masui, A., Bozano, G.C. Floriani, A. (2011). Nymphalidae. Part IV. Subfamily Apaturinae. In: Bozano, G.C. (Ed.) Guide to the Butterflies of the Palaearctic Region. Milano, Omnes Artes, 14, 1-82.

Ménétriés, E. (1832). Lépidoptères. In: Catalogue raisonné des objets de zoologie recueillis dans un voyage au Caucase et jusqu'aux frontières actuelles de la Perse entrepris par ordre de S. M. I'Empereur. St. Pétersbourg: L'Académie Impériale des Sciences, 241-268. https://doi.org/10.5962/bhl.title.63878

Ménétriés, E. (1855). Catalogue de la collection entomologique de L'Académie Impériale des Sciences de St.-Pétersbourg. Lépidoptères. I Partie: Les Diurnes. In: Enumeratio comporum animaluim Misei Imperialis Academiae Scientiarum Petropolitanae. Classis Insectorum ordo Lepidopterorum. Pars I. Lepidoptera Diurna, Petropoli, Typis Acad. Sci. Imp., 1-66. https://doi.org/10.5962/bhl.title.12490

Ménétriés, E. (1859). Verzeichniss von Insekten aus Gegend von Nachitschewan und dem nördlichen Persien. Nouv. Mén. Sco. Nat. Moscou 12, 247-248.

Murzin, V.S. (2000a). Family Danaidae. In: Guide to the Butterflies of Russia and Adjacent Territories (Lepidoptera, Rhopalocera). Pensoft, Sofia-Moscow 2, 10-11.

Murzin, V.S. (2000b). Family Nymphalidae: Limenitis, Seokia, Neptis, Aglais. In: Guide to the Butterflies of Russia and Adjacent Territories (Lepidoptera, Rhopalocera). Pensoft, Sofia-Moscow, 2, 16-23, 29-31.

Nekrutenko, Y.P. (1966). Eine neue Subspecies von Gonepteryx rhamni L.aus dem Kaukasus (Lep.Pieridae). Zt. Wien. Ent. Ges., 51, 44-47.

Nekrutenko, Y.P. (1968). Phylogeny and distribution of Gonepteryx (Lepidoptera, Pieridae). Naukova Dumka, Kiyv. (in Russian).

Nekrutenko, Y.P. (1972). A new subspecies of Eumedonia eumedon (Lycaenidae) from Caucasus. J. Lep. Soc., 26(4), 215218.

Nekrutenko, Y.P. (1977). A new subspecies of Heodes (Thersamonia) ochimus (Lepidoptera, Lycaenidae) from the Caucasus Minor. Dopovidi Academy of Science of Ukrainian SSR, series B, (5), 457-460 (in Ukranian).

Nekrutenko, Y.P. (1980). Revisional notes on lycaenid butterfly species assigned to Ultraaricia Beuret (Lycaenidae). Nota lepidopterologica, 3 (1-2), 55-68.

Nekrutenko, Y.P. (1985a). Lycaenids of the genus Vacciniina (Lepidoptera, Lycaenidae) in Transcaucasia. Vest. zool., 2, 87 (Russian).

Nekrutenko, Y.P. (1985b). New Blue Butterfly Taxa (Lepidoptera, Lycaenidae) from Transcaucasia and Middle Asia. Vest. zool., 4, 29-35 (Russian).

Nekrutenko, Y.P. (1989). New Satyrid Butterfly Taxa (Lepidoptera, Satyridae) from Zangezur Mountain Range. Vest. zool., 1, 14-18 (Russian).

Nekrutenko, Y.P. (1990). Butterflies of Caucasus. Naukova Dumka, Kiev (in Russian).

Nekrutenko, Y.P., Effendi, R.E. (1980). A new species of Tomares from Talysh Mountains (Lycaenidae). Nora Lepid., 3(1/2), 69-72.

Nekrutenko. Y.P., Effendi, R.E. (1983). A review of the blue butterflies of the group Lycaena phoenicurus Ld. (Lepidoptera, Lycaenidae) with description of a new species from Azerbaidzhan. Vestnik zoologii, 4, 8-15 (in Russian).

Nordmann, A. (1851). Die im Gebiete der Fauna Taurico-Caucasica beobachteten Schmetterlinge. Bull. Soc. Nat. Mosc., 24(2), 359-368, 24(3), 395-428.

Olivier, A., Puplesiene, J., van der Poorten, De Prins, W., Wiemers, M. (1999). Revision of some taxa of the Polyommaus (Agrodiaetus) transcaspicus group with description of a new species from Central Anatolia (Lepidoptera: Lycaenidae). Phegea, 27(1), 1-24.

Peile, H. (1922). The butterflies of Mesopotamia. J. Bombay net. Hist. Soc., 28(2), 243-267, 345-369.

Radde, G. (1899). Lepidoptera Caucasuca. In: Museum Caucaicum: Die Sammlungen des Kaukasischen Museums, Tiflis, Typ. Kanz. Landeschefs, 1, 419-422.

Reissinger, E. (1989). Die geographisch-subspezifische Gleiderung von Colias alfacariensis Ribbe, 1905 unter Berücksichtigung der Migrationsverhältnisse (Lepidoptera, Pieridae). Neue Ent. Nachr., 26, 1-351. 
Riley, N.D. (1939). Notes on oriental Theclinae (Lep.Lycaenidae) with descriptions of new species. Ann. Mag. nat. Hist., Ser. (9)8, 590-600.

Rjabov, M.A. (1958). Lepidoptera. In: Fauna of the USSR. Mountain Areas of the Europenean Part of USSR, 5, $351-375$. Romanoff, N.M. (1884-1887). Les Lépidoptères de la Transcaucasie. Romanoff, Mém. Lépid., 1, 1-92; 2, 1-118; 3, 1-49. Rühl, F. (1893). Tagfalter. In: Die palaearktischen Grosschmetterlinge und ihre naturgeschichte. Leipzig, E.Heyne, 1, 1-384. Samodurov, G.D. (2000). Family Lycaenidae (Part.). In: Guide to the Butterflies of Russia and Adjacent Territories (Lepidoptera, Rhopalocera). Pensoft, Sofia-Moscow, 2, 123-127, 177-182.

Samodurov, G.D., Korolew, V.A., Tshikolovets, V.V. (1996). Neue Taxa der Satyrinen-Gattung Hypobephele. Muschamp, 1915 (Lepidoptera: Nymphalidae, Satyrinae) aus Mittelasien und Transkaukasien. Nachr. entomol. Ver. Apollo, N.F. 17(1), 21-40.

Samodurow, G.D., Tschikolovets, V.V., Korolew, V.A. (2001). Eine Übersicht über die Satyriden der Gattung. Hyponephele Muschamp, 1915. VII. Die Arten Hyponephele lycaon (Rottemburg, 1775), H.pasimelas (Staudinger, 1886), H.lycanoides D. Weiss., Zhdanko, 1994, H.dzhungarica Samodurov, 1996, H.galtscha (Grum-Grshimailo, 1893) und H.lupina (Costa, 1836) (Lepidoptera, Satyridae). Atalanta, Würzburg, 32(1/2), 111-186.

Samodurov, G.D., Zhdanko, A.B. (2000). Family Lycaenidae: Thersamonia, Hyrcanana. In: Guide to the Butterflies of Russia and Adjacent Territories (Lepidoptera, Rhopalocera). Pensoft, Sofia-Moscow, 2, 127-130, 135-136.

Samodurov, G.D., Zhdanko, A.V., Tuzov, V.K. (2000). Family Lycaenidae: Plebejides. In: Guide to the Butterflies of Russia and Adjacent Territories (Lepidoptera, Rhopalocera). Pensoft, Sofia-Moscow, 2, 169-171.

Schurian, K.G. (1989). Ergänzungen und Berichtigungen zu: “Neuinteilung des Subgenus Lysandra der Gattung

Polyommatus Latreille (Lepidoptera: Lycaenidae). Ent. Zt., (9921), 305-320.

Sheljuzhko, L. (1925). Eine neue rasse von Pararge climene Esp. Pararge climene Esp.tkatshukovi (subsp. nov.). Ent. Anz., 89-91.

Sheljuzhko, L. (1929). Eininge neie palaearktische Lepidopteren-Formen. Mitt. Munch. ent. Ges., 19(10-12), 347-362.

Sheljuzhko, L. (1937). Lepidopterologische Ergebnisse meiner Reise nach dem teberda-Gebiet (Nordwest-Kaukasus).

Forsetzung. In: Festschr. 60. Geburtstage von Prof. Dr. Embrik Strand, Riga 2, 322-354.

Skala, P., Weidenhoffer, Z. (2002). New taxa of the genus Plebeius from Iran (Lepidoptera, Lycaenidae). Linn. Belgica, 18(8), 405-420.

Sorimachi, Y. (2000). The Primer of Colias. Kitamoto, Japan, 3, 1-235 (Japanese).

Sovinsky, V. (1905). Description d"ume nouvelle form d"Euchloe cardamines L. (Lepidoptera, Pieridae), intéressante au point de vue phylogénétique, et renarques sur la var.phoenissa Kalchberg de la meme espèce. Rev. Russ. Ent., 5(3/4), 103107 (Russian).

Stauder, M. (1924). Neue Palaearktenformen II. Mitt. Munch. ent. Ges. 14, 59-66.

Standfuss, M. (1892). Lepidopterologisches. In: Romanoff, N.M. (ed.) Mémoires sur les.Lépidoptères. St. Pétersbourg, Impr. Stassuléwitch, 6, 659-669.

Staudinger, O. (1899). Ueber die Arten und Formen der Lycaena Damon-Gruppe. Dt. ent. Z. Iris, 12(1), $137-155$. Staudinger, O. (1901). Macrolepidoptera. In: Staudinger, O., Rebel, H. Catalog der Lepidopteren des palaearctischen Faunengebietes. Berlin, Friedlander \& Sohn. https://doi.org/10.5962/bhl.title.120482

Stichel, H. (1911). Lepidopterologische Ergebnisse einer Sammelreise der Gebrüder rangnow nach persien. Mit Neubeschreibungen von R.Püngeler, E. Strand und dem Autor. Z. Wiss. Ins. - Biol., 7, 5-8, 37-40, 73-77, 112-117, $160-167$. Ten Hagen, W., Shurian, K.G. (2001). Ein Beitrag zur Kenntnis von Plebeius (Vacciniina) morgianus (Kurby, 1871) (Lepidoptera, Lycaenidae). Nachr. ent. Ver. Apollo, Frankfurt/Main, N.F., 21(4), 193-200.

Tschikolovets, V.V. (2003). Butterflies of Eastern Europe, Urals and Caucasus. Kyiv-Brno, Publ. by the author, 1-176.

Tschikolovets, V.V. (2011). Butterflies of Europe and the Mediterranean area. Tshikolovets Publications, Pardubice, 544 pp. Tshikolovets, V. \& Kostjuk, I. (1994) A catalogue of the type - specimens of Colias in the Zoological Museum of the Kiev University (Lepidoptera, Pieridae). Tshikolovets Press, Kyiv.

Tschikolovets, V.V., Nekrutenko, Y.P. (2012). The Butterflies of Caucasus and Transcaucasia (Armenia, Azerbaijam Georgia and Russian Federation). Tshikolovets Press, Kyiv-Pardubice.

Tomonaga, M. (1998). Anthocharis of the world. Yadoriga, 176, 8-19.

Turlin, B., Manil, L. (2005). Etude synoptique et Réoartition mondiale des Espéces du Genre Parnassius Latreille 1804 (Lepidoptera Papilionidae). Bulletin des Lépidoptéristes Parisiens-/le-de-France, 1-80.

Tuzov, V.K. (1993). The synonymic list of butterflies from the ex-USSR. Rosagroservice, Moscow.

Tuzov, V.K. (1997). Pieridae. In: Guide to the Butterflies of Russia and Adjacent Territories (Lepidoptera, Rhopalocera). Pensoft, Sofia-Moscow 2, 153-181.

Tuzov, V.K. (2000a). Family Lycaenidae (Part). In: Guide to the Butterflies of Russia and Adjacent Territories (Lepidoptera, Rhopalocera). Pensoft, Sofia-Moscow, 2, 101-185.

Tuzov, V.K. (2000b). List of illustrations. In: Guide to the Butterflies of Russia and Adjacent Territories (Lepidoptera, Rhopalocera). Pensoft, Sofia-Moscow, 2, 401-472.

Tuzov, V.K. (2000c). Family Nymphalidae (Part). In: Guide to the Butterflies of Russia and Adjacent Territories (Lepidoptera, Rhopalocera). Pensoft, Sofia-Moscow, 2, 9.

Tuzov, V.K., Zhdanko A.B., Dantchenko, A.V. (2000). Family Lycaenidae: Polyommatus. In: Guide to the Butterflies of Russia and Adjacent Territories (Lepidoptera, Rhopalocera). Pensoft, Sofia-Moscow, 2, 190-196. 
Van Oorscht, H., Wagner, S. (2000). Zu Tomares in der Türkei. Ergänzungen und Korrekturen zu Hesselbarth, van Oorschot \& Wagener, 1995: Die Tagfalter der Türkey. 3 (Lepidoptera). Phegea, 28(3), 87-117.

Verhulst, J. (2000). Les Colias du Globe/Monograph of the genus Colias. Goecke \& Evers, Keltern, 1-263.

Verity, R. (1905-1911). Rhopalocera palaearctica. [1]. Papilionidae et Rieridae. Firenze, Publ.by the author.

Verity, R. (1937). Des variations geographiques de I"Eumenis briseis L. Lambillionea, 37(1), 4-14; 37(3).

Vila, R., Lukhtanov, V.A., Talaverta, G., Gil-T. , Pierce, N.E. (2010). How common are dot-like distributions? Taxonomical oversplitting in western European Agrodiaetus (Lepidoptera: Lycaenidae) revealed by chromosomal and molecular markers. Biolog. J. Linn. Soc., 101, 130-154. https://doi.org/10.1111/j.1095-8312.2010.01481.x

Wagner, S. (1975). 4. Beitrag: Die vorderasiatischen Formen der Melanargia russiae (Esper, 1784) (Lepidoptera, Satyridae). In: Beitrage zun kenntnis der Rhopaloceren Irans. J. Ent. Soc. Iran, Suppl., 1, 47-60.

Weidenhoffer, Z. (2004). Subgenera Neolycaena, Superflua, Armenia, Rhymnaria (part). In: Weidenhoffer, Z., Bozano, G.C., Churkin, S. Lycaenidae. Part II. Subfamily Theclinae, Tribe Eumaeini (Partim): Satyrium, Superflua, Armenia, Neolycaena, Rhymnaria. In: Bozano, G.C. (Ed.). Guide to the Butterflies of the Palearctic Region. Milano, Omnes Artes, 7, 1-94.

Weidenhoffer, Z., Klir, J. (2000). A new species of Plebejus Kluk, 1780 from Iran (Lepidoptera, Lycaenidae). Linneana Belg, 17 (7), 289-292.

Zhdanko, A.B. (1993). Systematics, biology and distribution of blue butterflies of the genus Athamanthia Zhd.

(Lepidoptera, Lycaenidae). Entomologicheskoe Obozrenie, 72 (3), 664-674 (in Russian).

Zhdanko, A.B. (1997). On nomenclature of some Palaearctic Lycaenidae (Lepidoptera). Zoosystematica Rossica, $5(2), 312$. Zhdanko, A.B. (1999). New species of the genera Callophrys Billb. and Polyommatus Latr. (Lepidoptera, Lycaenidae) from Asia and Caucasus. Vestn. KazGU, ser. Biol., 5, 46-52 (Russian).

Zhdanko, A.B. (2000). Family Lycaenidae (Part). In: Guide to the Butterflies of Russia and Adjacent Territories (Lepidoptera, Rhopalocera). Pensoft, Sofia-Moscow, 2, 102-184.

Zhdanko, A.B., (2002). An annotated list of species of the family Lycaenidae (Lepidoptera) occurring in Kazakhstan. Tethys entomol. Research, 4,125-146.

\section{Citation:}

Snegovaya, N.Y., Petrov, V.A. (2019). A catalogue of butterflies (Lepidoptera, Rhopalocera) of Azerbaijan Acta Biologica Sibirica, 5 (3), 62-117.

Submitted: 14.07.2019. Accepted: 16.09.2019

cross ref http://dx.doi.org/10.14258/abs.v5.i3.6433

(C) 2019 by the authors. Submitted for possible open access publication under the terms and conditions of the

Creative Commons Attribution (CC BY) license (http://creativecommons.org/licenses/by/4.0/). 